\title{
Mechanistic Analysis of Double-Shell Tank Gas Release
}

Progress Report - November 1990
R. T. Allemann
C. E. Haines
Z. I. Antoniak
L. M. Liljegren
J. R. Friley
S. Somasundaram

December 1991

Prepared for the U.S. Department of Energy under Contract DE-AC06-76RLO 1830

Pacific Northwest Laboratory Operated for the U.S. Department of Energy by Battelle Memorial Institute 


\section{DISCLAIMER}

This report was prepared as an account of work sponsored by an agency of the United States Government. Neither the United States Government nor any agency thereof, nor Battelle Memorial Instilute, nor any of their employees, makes any warranty, expressed or implied, or assumes any legal liability or responsibility for the accuracy, completeness, or usefulness of any information, apparatus, product, or process disclosed, or represents that its use would not infringe privately o: wned rights. Reference herein to any specific commercial product, process, or servica by trade name, trademark, manufacturer, or otherwise does not necessarily constitu! $\circ$ or imply its endorsement, recommendation, or favoring by the United States Government or any agency thereof, or Baltelle Memorial Institute. The views and opinions of authors expressed herein do not necessarily state or reflect those of the United States Government or any agency thereof.

\section{PACIFIC NORTHWEST LABORATORY}

operated by

BATTELLE MEMORIAL INSTITUTE

for the

UNITED STATES DEPARTMENT OF ENERGY

under Contract DE-ACO6-76RLO 1830

Printed in the United States of America

Available to DOE and DOE contractors from the

Office of Scientific and Technical Information, P.O. Box 62, Oak Ridge, TN 37831; prices available from (615) 576-8401. FTS 626-8401.

Available to the public from the National Technical Information Service,

U.S. Department of Commerce, 5285 Port Royal Rd., Springfield, VA 22161. 
MECHANISTIC ANALYSIS OF DOUBLESHELL TANK GAS RELEASE

PROGRESS REPORT--NOVEMBER 1990
R. T. Allemann
Z. I. Antoniak
J. R. Friley
C. E. Haines
L. M. Liljegren
S. Somasundaram

December 1991

Prepared for the U.S. Department of Energy under Contract DE-AC06-76RLO 1830

Pacific Northwest Laboratory

Richland, Washington 99352 


\section{SUMMARY}

Pacific Northwest Laboratory (PNL) $)^{(a)}$ is studying possible mechanisms and fluid dynamics contributing to the periodic release of gases from the doubie-shell waste storage tanks at Hanford. This study is being conducted for Westinghouse Hanford Company (WHC), a contractor for the U.S. Department of Energy (DOE). This interim report discusses the work done through November 1990.

The approach is

- to assemble and compile data that are pertinent to the mechanisms

- to analyze the data

- to evaluate physical properties and parameters

- to evaluate hypothetical mechanisms

- to develop mathematical models of the mechanisms.

The accomplishments to daie are summarized below.

- Several gas release hypotheses have been characterized and analyses begun to see if the hypotheses agree with observations.

- Some analysis has essentially eliminated the idea that gas is held by the crust and released when the crust fractures. This mechanism fails to explain the temperature profile changes. Data on the strength of the crust material are not available, but estimates and photo observations suggest that the crust would not have the strength or seal necessary to contain the gas.

- The temperature profiles have been assembled. Profile changes have represented a moving target for the analysis. Each new gas release has been accompanied by a new set of data of varying accuracy and completeness.

- A model has been developed for the size and distribution of bubbles held in the sludge. However, the hypothesis of gas release from the bottom sludge, which required calculations on the size and mobility of bubbles, has been called into question by the temperature data of the release of October 24, 1990. The data show a temperature

(a) Pacific Northwest Laboratory is operated for the U.S. Department of Energy by Battelle Memorial Institute under Contract DE-AC06-76RLO 1830. 
profile "flip-flop" and suggest a gross turnover of the bottom sludge layer rather than a bubble release. Unfortunately, the calculations that were based on the specific gravities of samples taken in 1987 preclude the turnover mechanism. At this time the accuracies of the specific gravity of those samples are suspect. A water balance was not obtained for the middle samiple, which was less dense than the sample above it.

- The same samples gave composition data that were used to calculate the heat output of the tank. The heat output was calculated for the different layers, and suggests that about half the heat comes from the bottom layer and that about $5 \%$ arises in the crust.

- Photo analysis of the lances and thermocouple trees that are bent shows that they are not bent to the same degree or in the same tangential direction. This indicates that the crust does not move as a unit, and implies that cracking and motion of different crust plates must have occurred, perhaps like continental drift, having subduction to accommodate the motion. The forces that move the plates are derived from the energy of the buoyant gases.

- Although the motion of the crust and the fluids may be chaotic, the fundamental patterns are being studied through stability analysis of fluid layers and fluid dynamic modeling to see if the situation is stabilized by viscosity or interface tension of the layers. 


\section{CONTENTS}

SUMMARY ..................................

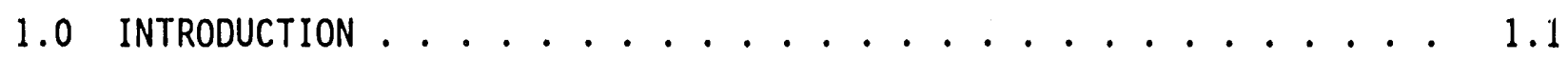

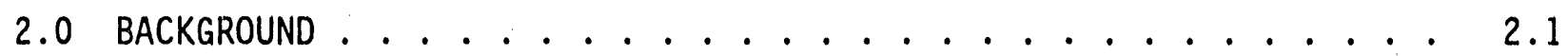

2.1 CHANGES IN LIQUID LEVELS . . . . . . . . . . . 2.1

2.2 TEMPERATURE CHANGES ASSOCIATED WITH THE LEVEL DROPS . . . . 2.11

2.3 PRESSURIZATION CHANGES IN THE TANK HEAD SPACE . . . . . . 2.15

2.4 COMPOSITION AND VOLUME OF GAS RELEASES . . . . . . . 2.16

2.5 DURATION OF THE GAS RELEASE EVENTS . . . . . . . . 2.17

2.6.1 Chemical Composition of the Tank Contents ..... 2.19

2.6.2 Rheology of the Tank Contents ......... . 2.23

2.7 DATA ON SLURRY GROWTH STUDIES USING SIMULATED SLURRIES $\ldots \quad .27$

2.8 COMPARISON OF THE RECENT GAS-RELEASE EVENTS . . . . . . 2.32

2.8.1 Special Features of the Gas Releases........ 2.32

2.8.2 Flow Rate Variation ............ 2.32

2.8.3 Pressure Variation . . . . . . . . . 2.32

2.9 HEAT TRANSFER PROPERTIES AND ANALYSES . . . . . . . 2.33

2.9.1 Natural Convection Heat Transfer ......... 2.34

2.9.2 Thermal Conductivity of Slurry/Bubble Mixture . . . 2.35

2.9.3 Thermal Conductivity of Slurry . . . . . . . . 2.37

2.10 PHOTOGRAPH DESCRIPTION AND ANALYSIS . . . . . . . 2.38

2.10.1 Photographic Analysis Procedure ........ 2.38

2.10.2 Thermocouple Probe Elevations .......... 2.40 
3.0 RECOMMENDATIONS ON DATA GATHERING .............. 3.1

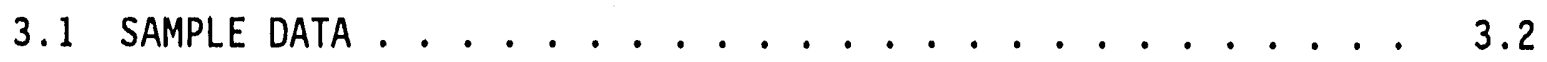

3.2 PHOTOGRAPHY . . . . . . . . . . . . . . . 3.2

4.0 DEFINITIONS OF MECHANISTIC MODELS FOR TANK 101-SY

GAS RELEASE ............................ 4.1

4.1 MODELS FOR GAS HELD BY THE CRUST . . . . . . . . . . 4.1

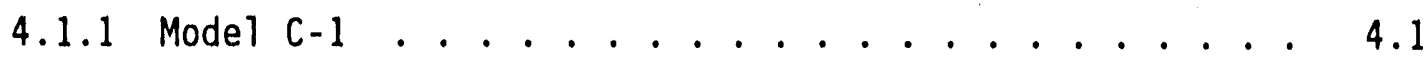

4.1 .2 Model $C-2 \ldots . . . . . . . . .44$

4.2 MODELS FOR GAS HELD IN SLUDGE . . . . . . . . . 4.2

4.2 .1 Model R-1 ............... 4.2

4.2 .2 Model R-2 ............... 4.2

4.2 .3 Model R-3 ............... 4.3

4.2 .4 Model R-4 ................ . . 4.3

4.2 .5 Model R-5 ............... 4.3

4.2 .6 Model R-6 . . . . . . . . . . . . 4.4

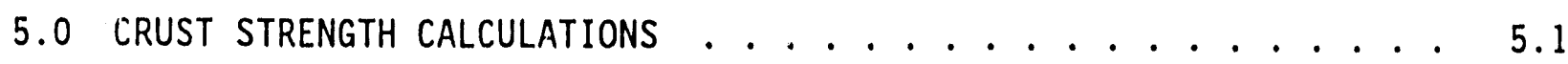

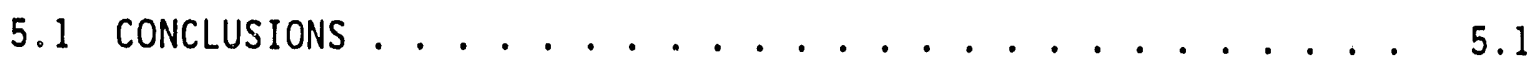

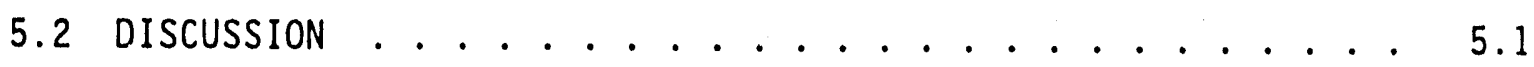

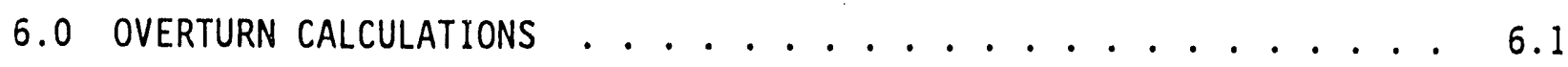

6.1 SUMMARY $\ldots \ldots \ldots \ldots . \ldots . \ldots . \ldots . \ldots . \ldots$

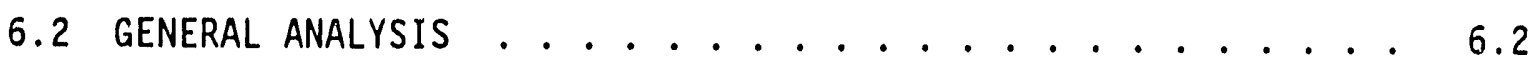

6.2.1 Assumptions Based on Published Tank Data . . . . . . 6.5

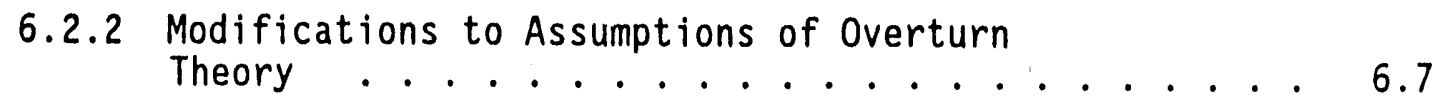

6.3 EFFECT OF HYDROSTATIC PRESSURE VARIATION . . . . . . 6.9

7.0 HYDROGEN BUBbLE FORMATION AND SIZE . . . . . . . . . . . 7.1

8.0 DESCRIPTIVE MODEL FOR BUBBLE SIZE AND MOTION . . . . . . . . 8.1 


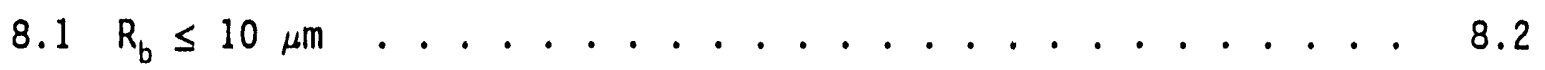

$8.210 \mu \mathrm{m}<\mathrm{R}_{\mathrm{b}} \leq 50 \mu \mathrm{m} \ldots \ldots . \ldots . \ldots . \ldots . \ldots . \ldots . . \ldots$

$8.350 \mu \mathrm{m}<\mathrm{R}_{\mathrm{b}} \leq 100 \mu \mathrm{m} \ldots \ldots \ldots . \ldots . \ldots . \ldots . \ldots . \ldots$

$8.4100 \mu \mathrm{m}<\mathrm{R}_{\mathrm{b}} \leq 500 \mu \mathrm{m} \ldots \ldots . \ldots . \ldots . \ldots . \ldots . \ldots . \ldots$

$8.5500 \mu \mathrm{m}<\mathrm{R}_{\mathrm{b}} \leq 2000 \mu \mathrm{m} \ldots \ldots . \ldots . \ldots . . \ldots 8$

$8.60 .2 \mathrm{~cm}<\mathrm{R}_{\mathrm{b}} \leq 0.3 \mathrm{~cm} \ldots \ldots . \ldots . . \ldots . . \ldots 8$

9.0 DISCUSSION OF VOLUME AND LOCATION OF GAS . . . . . . . . . . 9.1

9.1 CRUST LEVEL DROP AND PRESSURE RISE . . . . . . . . . . . 9.1

9.2 INCREASE IN VENT FlOW RATE ................ 9.2

9.3 LOCATION AND STATE OF THE GASES . . . . . . . . . . . 9.3

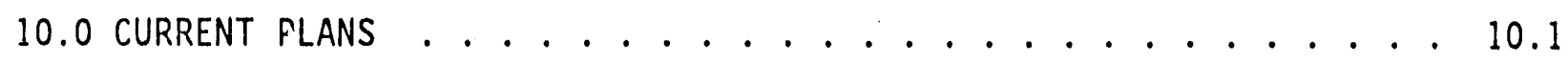
11.0 REFERENCES . . . . . . . . . . . . . . . . . 11.1 APPENDIX A - EFFECT OF DILATION ON DIFFUSION TO BUBBLES IN MATRIX . . . A.1 APPENDIX B - CALCULATION OF BUBBLE NUMBER DENSITY . . . . . . . . . B.I APPENDIX C - ESTIMATION OF PARAMETERS . . . . . . . . . . . . c.1

APPENDIX D - ESTIMATION OF HEAT PRODUCTION OF TANK 101-SY ...... D.1 


\section{FIGURES}

2.1 Tank 101-SY Riser Locations . . . . . . . . . . . . 2.4

2.2 Liquid Levels in Tank 101-SY During Gas Releases . . . . . . 2.6

2.3 Surface Level Readings of Tank 101-SY, October 24, $1990 \ldots 2.7$

2.4 Surface Level Readings of Tank 101-SY, August 5-7, 1990 . . . 2.8

2.5 Surface Level Readings of Tank 101-SY, 1981 to 1990 . . . . . 2.9

2.6 Relationship Between Temperatures at Thermocouples 2, 8,

2.7 April 19, 1990, Event of Tank 101-SY, 12-h Plot . . . . . 2.10

2.8 April 19, 1990, Event of Tank 101-SY, 2-h Plot . . . . . 2.11

2.9 Comparison of Profiles Measured Prior to Level Drops . . . . 2.13

2.10 Temperature Evolution Between Tank Level Drops . . . . . . 2.13

2.11 Temperatures Change for the Level Drop of December 1989 . . . . 2.14

2.12 Current Profile and Pre- and Post-August 5, 1990, Burp Profile ................. 2.15

2.13 Stress Versus Strain Curves for the Contents of Tank 101-SY, Top Sample.............. 2.24

2.14 Stress Versus Strain Curves for the Contents of Tank 101-SY, Middle Sample .................. 2.24

2.15 Stress Versus Strain Curves for the Contents of Tank 101-SY, Bottom Sample............... 2.25

2.16 Shear Stress Versus Time at Constant Strain Rate for a Hypothetical Tank Sludge .............. 2.26

2.17 Observed Relationship Between Tank Growth and the Initial Height of the Tank Contents ............. 2.3.

2.18 Observed Relationship Between Volume Growth and Tank Volume . . . 2.31

2.19 Effect of Tilt of Thermocouple Probe on Temperature Profile . . 2.41

5.1 Crust Stresses Due to Bottom Pressure . . . . . . . . . . . 5.3

6.1 Expanded Tank Configuration . . . . . . . . . . . . 6.3 
6.2 Unexpanded Tank Configuration ............. 6.3

7.1 Concentration of Gas Production Diffusing to Nucleated Bubble Sites ............... 7.2 


\section{TABLES}

2.1 Additions to Tank 101-SY . . . . . . . . . . . 2.3

2.2 Height Correction for Titled Thermocouple Probe . . . . . . 2.12

2.3 Summary of Gas Analysis Data . . . . . . . . . . . . 2.17

2.4 Estimated Composition of Tanks 101-SY and 103-SY Ba: 3d on

Additions Made Prior to September 1984 . . . . . . . . . . . 2.19

2.5 Composition of Tank 101-SY Reported by Mauss . . . . . . . . 2.22

2.6 Effective Viscosity of Undiluted Slurry Samples for

Tank 101-SY ................... 2.23

2.7 Data on Recent Gas Releases Tank 101-SY . . . . . . . . . . 2.33

2.8 Slurry Rayleigh Numbers . . . . . . . . . . . . . . 2.35

2.9 Tank 101-SY Heat Transfer Calculations . . . . . . . . 2.36

2.10 Waste Tank Photo Analysis . . . . . . . . . . . . 2.39

5.1 Material Properties and Geometry Nomericlature . . . . . . . . 5.2

6.1 Tank Heights and Specific Gravities Used to Test the Tank

Destabilization Theory ............ 6.7 


\subsection{INTRODUCTION}

Safe management of the wastes at Hanford depends on an understanding of the chemical and physical mechanisms that take place in the waste tanks. An example of the need to understand these mechanisms is tank 101-SY. The waste in this tank is generating and periodically releasing potentially flammable gases into the tank vent system according to observations of the tank. How these gases are generated and become trapped, the causes of periodic release, and the mechanism of the release are not known in detai1. In order to develop a safe mitigation strategy, possible physical mechanisms for the periodic release of $\mathrm{fl}$ ammable gases need to be understood.

As a part of the overall program, Westinghouse Hanford Company has commissioned Pacific Northwest Laboratory (PNL) to develop an understanding of the processes below the crust in the double-shell waste tanks (such as tank $101-\mathrm{SY}$ ) and to mathematically model and explain the physical mechanisms giving rise to the accumulation and periodic release of flammable gases from some of these waste tanks.

In the initial part of the work, PNL obtained and correlated data, analyzed data, compared data with expected physical properties, defined mechanisms, and prepared initial models of gas formation and retention. This is an interim report summarizing the status of the work done to date.

This report contains eleven sections. Section 2.0 provides background information. Recommendations on data gathering and definitions of mechanistic models are presented in Sections 3.0 and 4.0 , respectively. Section 5.0 is a discussion of crust strength calculations and Section 6.0 discusses overturn calculations. Sections 7.0 and 8.0 describe hydrogen bubble formation and a model for bubble size. A discussion of volume and location of gas is provided in Section 9.0 and Section 10.0 discusses current plans. 


\subsection{BACKGROUND}

Data describing the tank contents were collected including physical data describing the viscosity and chemical composition of the contents of tank 101-SY, of other tanks that exhibit slurry growth, and some physical data on synthetic slurries. Although the properties of synthetic slurries may not be identical to those in the waste tanks, some of the information gathered using these slurries provides a more detailed understanding of possible behaviors in real slurries. In particular, the data from synthetic slurries may provide some bounds for the gas generation rate in the tank. Data describing the changes that occur in tank 101-SY during tank level drops also were collected.

A number of documents (including unpublished letters and memos) describing the changes that occur during tank level drops were obtained; efforts to obtain additional data are ongoing. The data reported in these documents include:

1. measurements of the tank levels (Panjunen et al. 1990)

2. tank temperatures

3. measurements of the pressures in the tank head space (Panjunen et al. 1990)

4. measurements of the volume and composition of released gases (Panjunen et a1. 1990).

The data on these four topics are summarized in the following sections.

\subsection{CHANGES IN LIQUID LEVELS}

The content levels of a number of SX and SY tanks have been observed to fluctuate since at least June 15, 197\%. (a) At that time, periodic level fluctuations of 25 to $50 \mathrm{~mm}$ had been observed in a number of SY and SX tanks, and a calculation was performed to determine if the rate at which hydrogen was produced by radiolysis was sufficient to explain the level fluctuations in

(a) D. C. Lini, Atlantic Richfield Hanford Company, unpublished. 
tank 101-SX. (a) It is not clear whether tank 101-SY was on the 1ist of tanks that were exhibiting periodic level fluctuations at that time.

An internal letter dated October 29, 1983, reported that periodic growth and level drops occurred in tank 101-SY only; the letter stated that periodic level drops had been occurring in tank 101-SY for 3 years, or since 1980. The same letter indicated that level drops had been observed in tank 103-sY. It appears that large periodic fluctuations with level drops significantly greater than 1 to $2 \mathrm{in.} \mathrm{occur} \mathrm{only} \mathrm{in} \mathrm{tank} 101-\mathrm{sY}$. Other tanks exhibit smaller periodic level fluctuations. Surface level plots for tanks 101-SY, 103-SY, and 103-AN described in two informal communications written by a PNL researcher showed at least two unexplained level drops of approximately 2 in. in tank 103-SY between June 1988 and January 1990. Other level drops may have occurred, but are more difficult to interpret since they appear to occur in conjunction with transfers to the tank. It is possible that there are some qualitative similarities between the growth and low-magnitude periodic fluctuations discussed by Lini and those being studied in tank 101-SY.

A unique feature of tank 101-SY is that it contains both complex concentrate and doubie-shell slurry (DSS). Tank fill information indicates that at the time the letters by Lini and Strachan were written, tank 101-SY contained $101 \mathrm{in}$. of DSS. Waste produced from an evaporator run from tank 102-SY was added to tank 101-SY later that year. The dates on which additions were made to the tank are shown in Table 2.1. Additions made after 1984 are not accounted for in Table 2.1; it appears that 3 in. of water were added between September 1984 and May 1988. This would cause the total tank height to rise to $390 \mathrm{in}$. However, the volume of the tank contents would be expected to fall as a result of evaporation.

The rate of evaporation from the tank was estimated on the basis of psychometric data taken from an internal memo written by $D$. Reynolds in May 1990. The author of the memo concluded that water is removed by evaporation from the tank at a rate of from $350 \mathrm{lbm} /$ day on hot days when the relative humidity is

(a) D. M. Strachan, Atlantic Richfield Hanford Company, memo. 
IABLE 2.1. Additions to Tank 101-SY(a)

\begin{tabular}{lccc}
\multicolumn{1}{c}{ Fill Type } & Date & Inches Added \\
First DSS & $4 / 25 / 77$ & & 101.1 \\
Evaporator run from tank 102-SY & $11 / 1 / 77$ & 132.75 \\
Transfer from tank 106-SX & $6 / 25 / 78$ & 48.4 \\
Transfer from tank 111-U & $8 / 14 / 78$ & 21.6 \\
Second DSS & $10 / 29 / 80$ & & 84.0 \\
Total & & & 387.85
\end{tabular}

(a) Information taken from an internal letter written by G. P. Simpson of Rockwell International, dated August 28, 1984.

low to $44 \mathrm{lbm} /$ day on cold days when the relative humidity is high. The rate at which the tank is ventilated also affects the evaporation rate. Evaporation rates of this magnitude would cause the tank level to fall at a rate of approximately $0.6 \mathrm{in./yr}$ to $6 \mathrm{in./yr}$. However, no systematic decreases in the tank levels have been detected.

The accuracy with which the volume additions to tank 101-SY were measured and recorded is not known. It was noted in an internal memo (written by J. P. Harris III) that there are discrepancies in the level readings and fill records of other tanks and suggests that "discrepancies between their level readings and transfer volumes are probably due to accumulated math errors over time or misplaced transfer information during data gathering from historical files." Tank 101-SY is not discussed in this memo. However, the fact that the volume of tank contents cannot be reproduced on the basis of fill records indicates that fill information may not be complete and cannot be used to determine accurately the volume of slurry that was originally put in the tank.

Growth is reported to have been observed in tank 101-SY after both the first and second additions in 1977 (Delegard 1980). The reported growth is confirmed in a letter discussing historical tank growth that indicates that by July 1977 tank 101-SY had already experienced 3.5 in. of growth. At that time at least 11 tanks had exhibited growth, and a number had exhibited significantly more growth than tank 101-SY. Neither of these documents mentions periodic level changes. 
The liquid level in tank 101-SY is measured using two separate gages referred to in Rockwell International letters as the FIC (Food Instrument Corporation) and manual gages. Figure 2.1 illustrates the physical separation between the two instruments (diagram taken from Panjunen et a1. [1990]). The two instruments are reported to be separated by $45 \mathrm{ft}$; thus differences in the readings using the two instruments may result if the crust does not rise evenly.

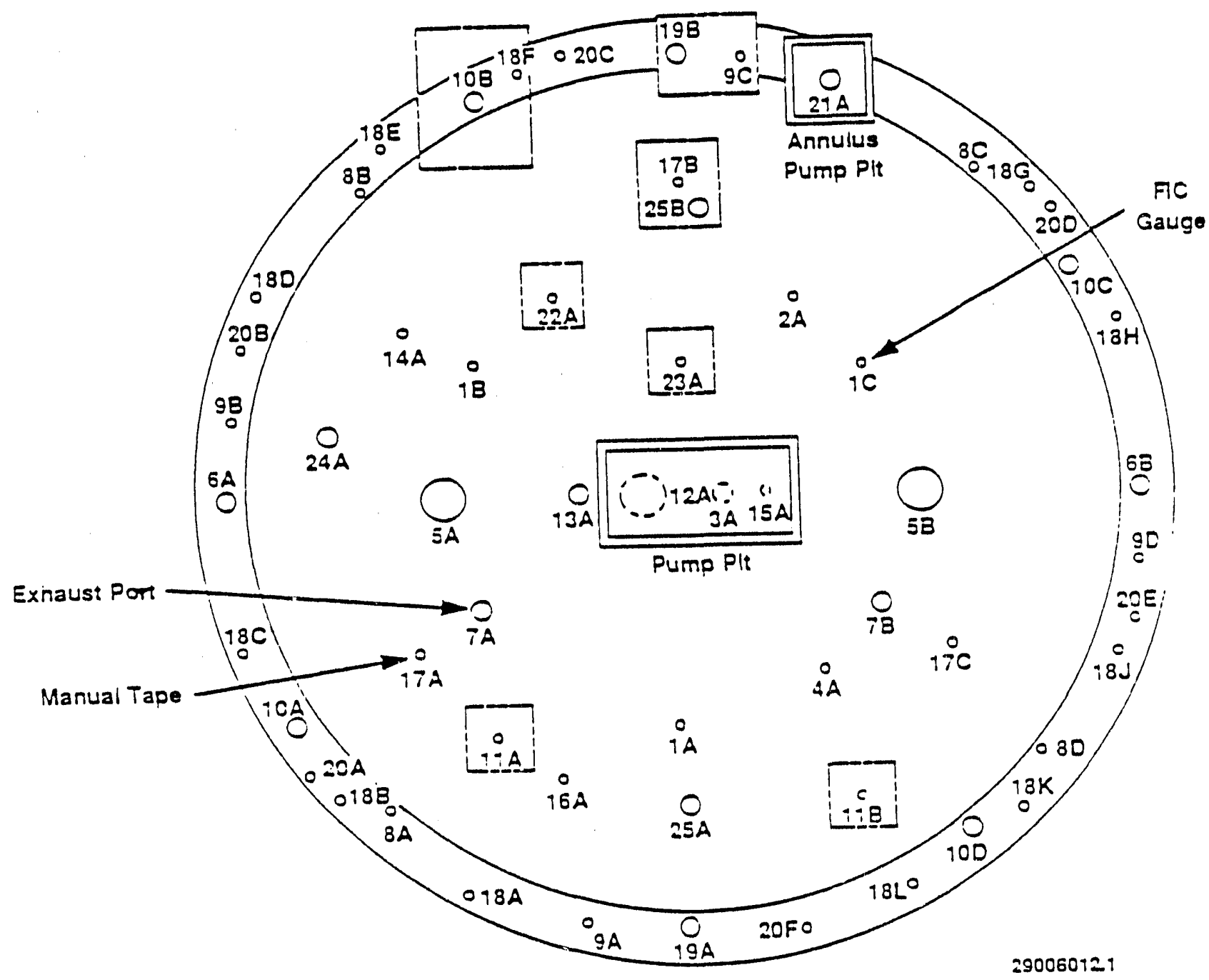

FIGURE 2.1. Tank 101-SY Riser Locations 
Alternatively, differences in level readiligs may be the result of error in the measurement, since the resolution of the instrument is not stated in any of the documents in which elevation levels are reported. Information describing the accuracy and resolution of a typical level gage was obtained in a telephone conversation with a Westinghouse Hanford employee who stated that the information given is not specific to tank 101-SY, but is typical of operation of most level gages. It appears that the resolution is $0.1 \mathrm{in.;}$ however, the accuracy is much lower.

Accuracy of the level measurement is affected by many factors, the two most important being 1) the monitor requires that the region of the crust in contact with the FIC sensor be slightly conductive (if not, the crust is not considered to have been reached, and no level reading is reported), and 2) it is possible for the monitor cable to go slack, in which case the level reading reported is lower than actually is the case. During periods when the crust level is rising, the monitor cable or tape would be expected to go slack, and a crust rise would not be detected. In order to avoid this, the method of operation calls for the monitor to be raised and lowered at intervals of approximately $1 \mathrm{~min}$. The location at which the crust is first contacted is determined and is reported at the beginning of each interval. Loss of contact could be detected if the crust fell; however, the slackening of the tape would not be detected if the crust rose. Consequently, the accuracy of the level reading in the middle of the time interval is not known. The exact time interval used in tank 101-SY was not known.

Changes in the level of the tank contents are reported for the drops that occurred between January 1985 and July 1985. (a) A number of these level drops occurred as a result of tank lancing. Readings from both instruments are shown in Figure 2.2 and suggest that the crust does not rise and fall evenly. The FIC gage indicates a higher level during most of the time periods; however, the manual gage sometimes records a higher reading. Thus the crust appears to rise unevenly, and the location of the high point changes from time to time.

(a) L. M. Sasaki, Rockwe11 International, internal letter. 


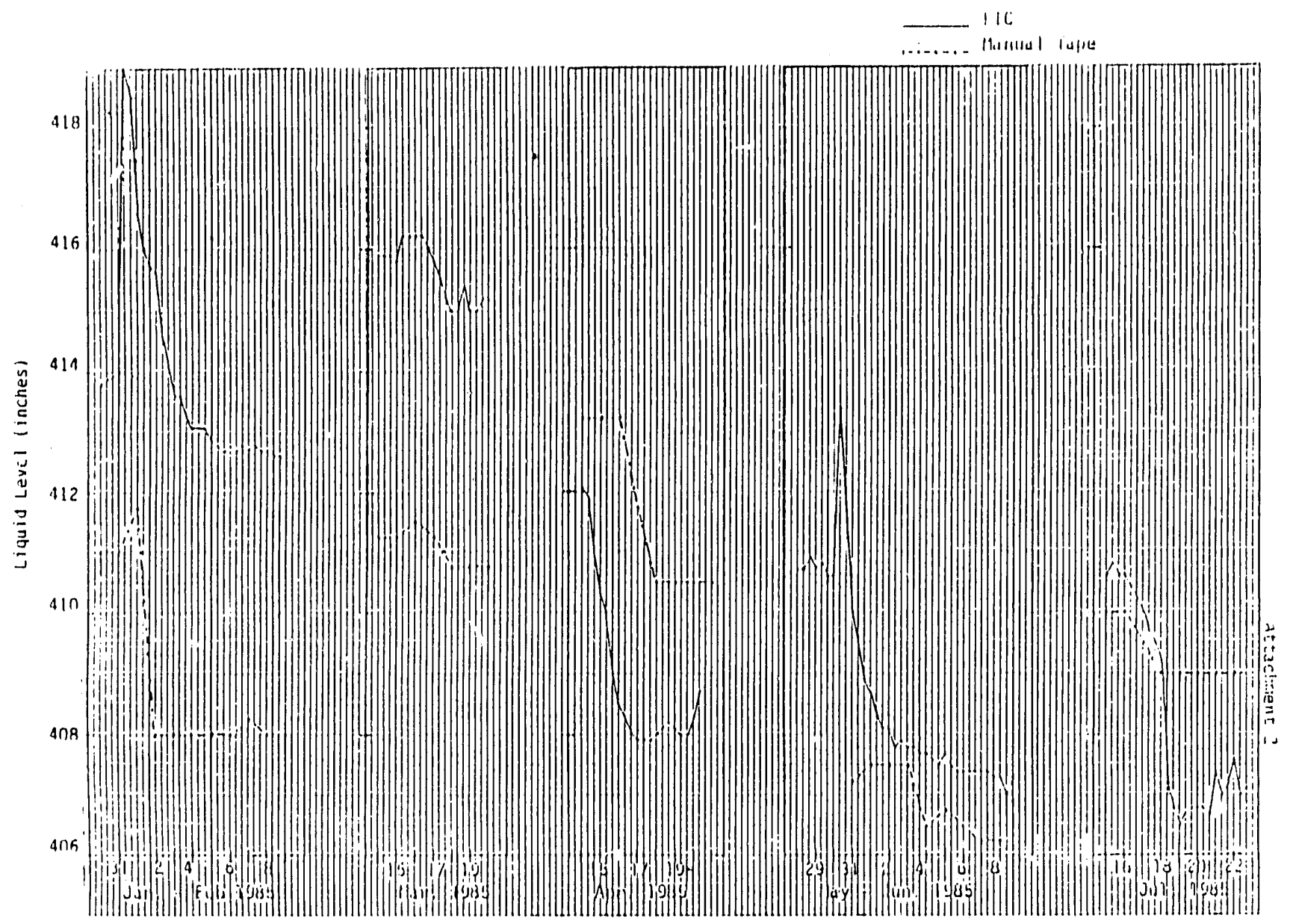

FIGURE 2.2. Liquid Levels in Tank 101-SY During Gas Releases

Sasaki also notes that the level drops are often preceded by a sharp rise in the tank level contents. This "prelude rise" appears to have occurred before the level drops in January and May 1985. Less pronounced prelude rises appear to have occurred in March and July 1985. The level drop in April 1985 does not appear to have been preceded by any rise in the level of the tank contents. A prelude rise in the tank level contents might be consistent with compressed gases released from the lower regions in the tank expanding as they rise through the waste.

It is not known if the prelude rise in the level of the tank contents occurs with every level drop, or if it is an occasional occurrence. A prelude 
rise was also observed in the most recent level drop that occurred on october 24, 1990 (Figure 2.3). It had a very short duration and was only detected during one measurement cycle by the FIC gage. Changes in the tank level during the period between August 5, 1990, and August 7, 1990, are shown in Figure 2.4. The level change during the August gas release was approximately 5 in. No prelude rise in the tank level was observed. Because the FIC gage response rate is 1 imited by the time required to raise and lower the gage, it is possible that very rapid fluctuations in the level of the tank contents would not be observed. In addition, the spike would generally not be detected when measurements are taken at longer intervals.

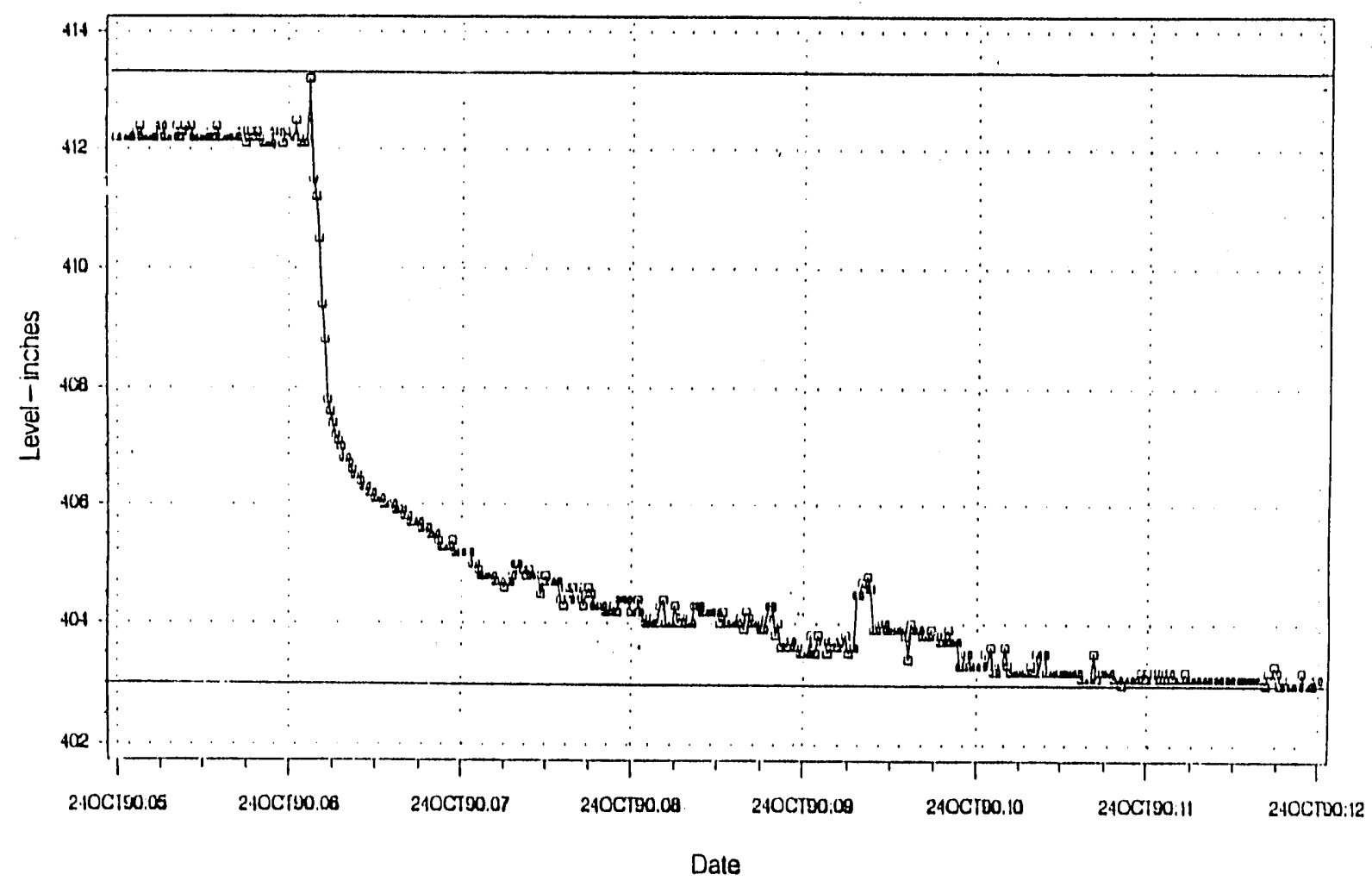

FIGURE 2.3. Surface Level Readings of Tank 101-SY, October 24, 1990 


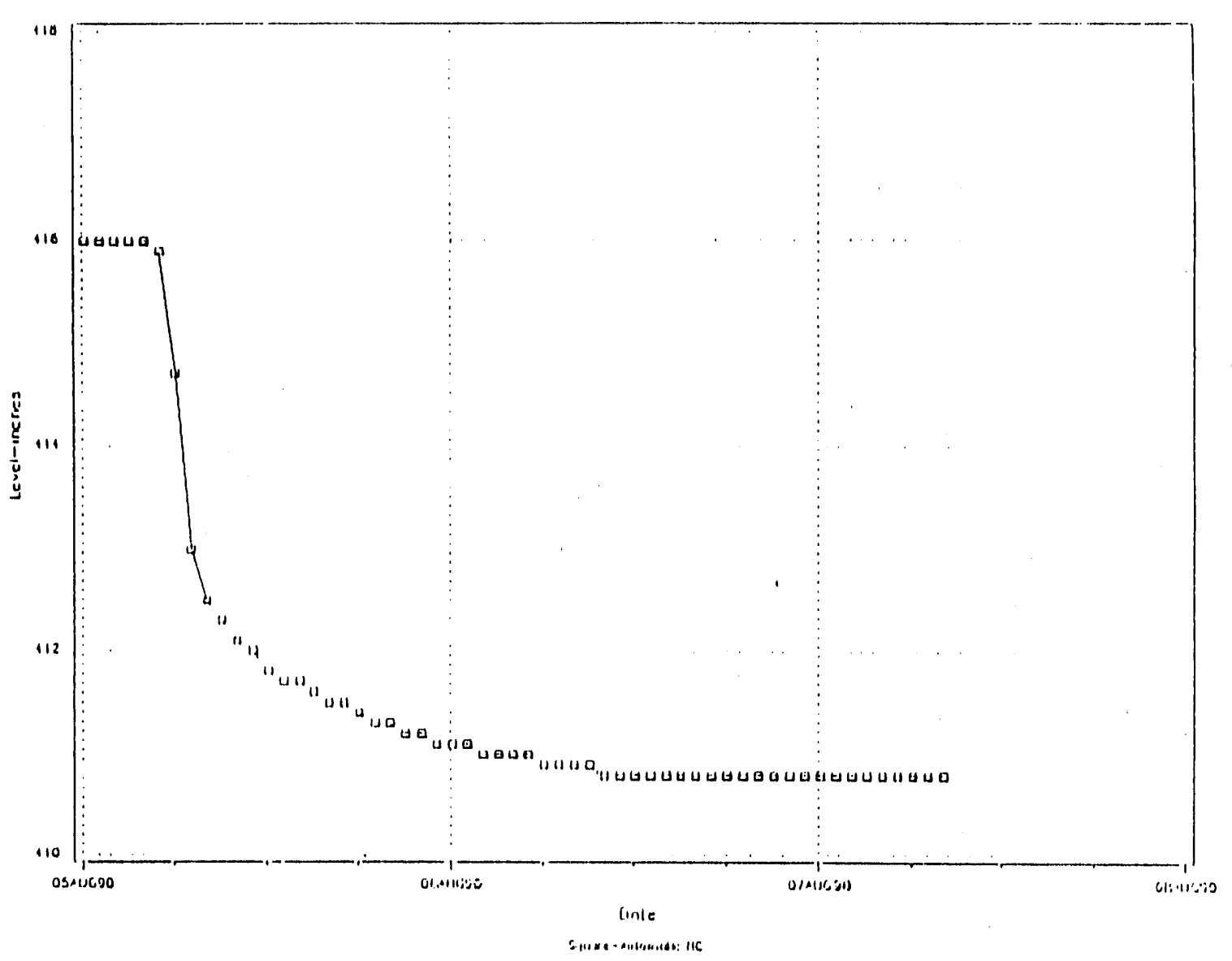

FIGURE 2.4. Surface Level Readings of Tank 101-SY, August 5-7, 1990

The liquid level measurements taken during the period from 1981 to April 1990 are reported by Panjunen et al. (1990) and shown in Figure 2.5. (a)

During this period, the maximum tank level was 423 in. and the minimum level was $403 \mathrm{in.}$ The frequency of level drops decreased somewhat with time, while the magnitude of the level drops appeared to be increasing. Changes in the tank level during the period from December 24, 1989, to April 30, 1990, were noted in a memo by Dan Reynolds and are shown in Figure 2.6. During this period, the tank level reached a maximum of 417 in. and a minimum of 408 in.

(a) There appears to be some discrepancy between the tank level data noted in Sasaki's letter for the period between January 1985 and July 1985 and the data for the similar period reported by Panjunen. This discrepancy has not been investigated. 


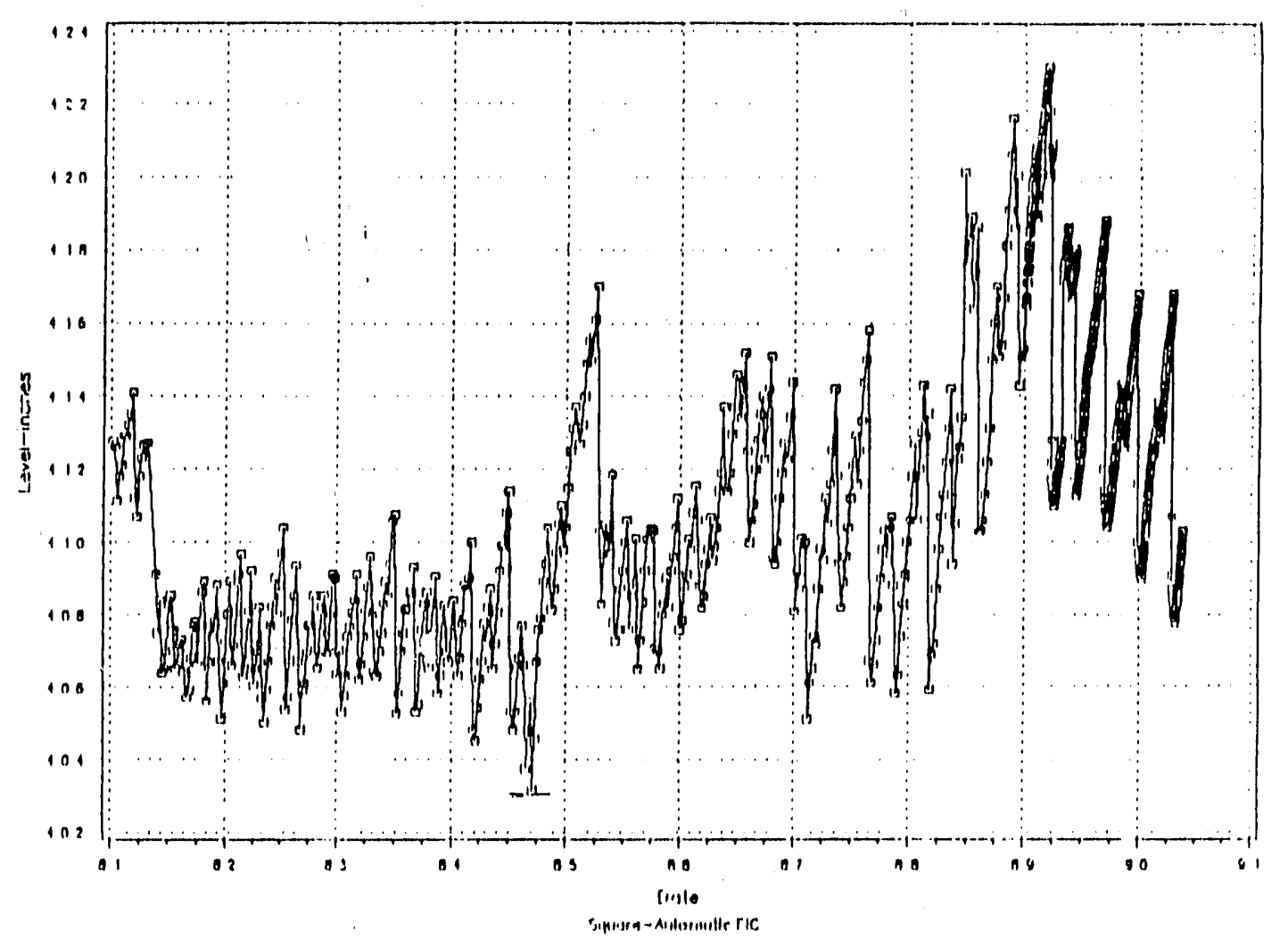

FIGURE 2.5. Surface Level Readings of Tank 101-SY, 1981 to 1990

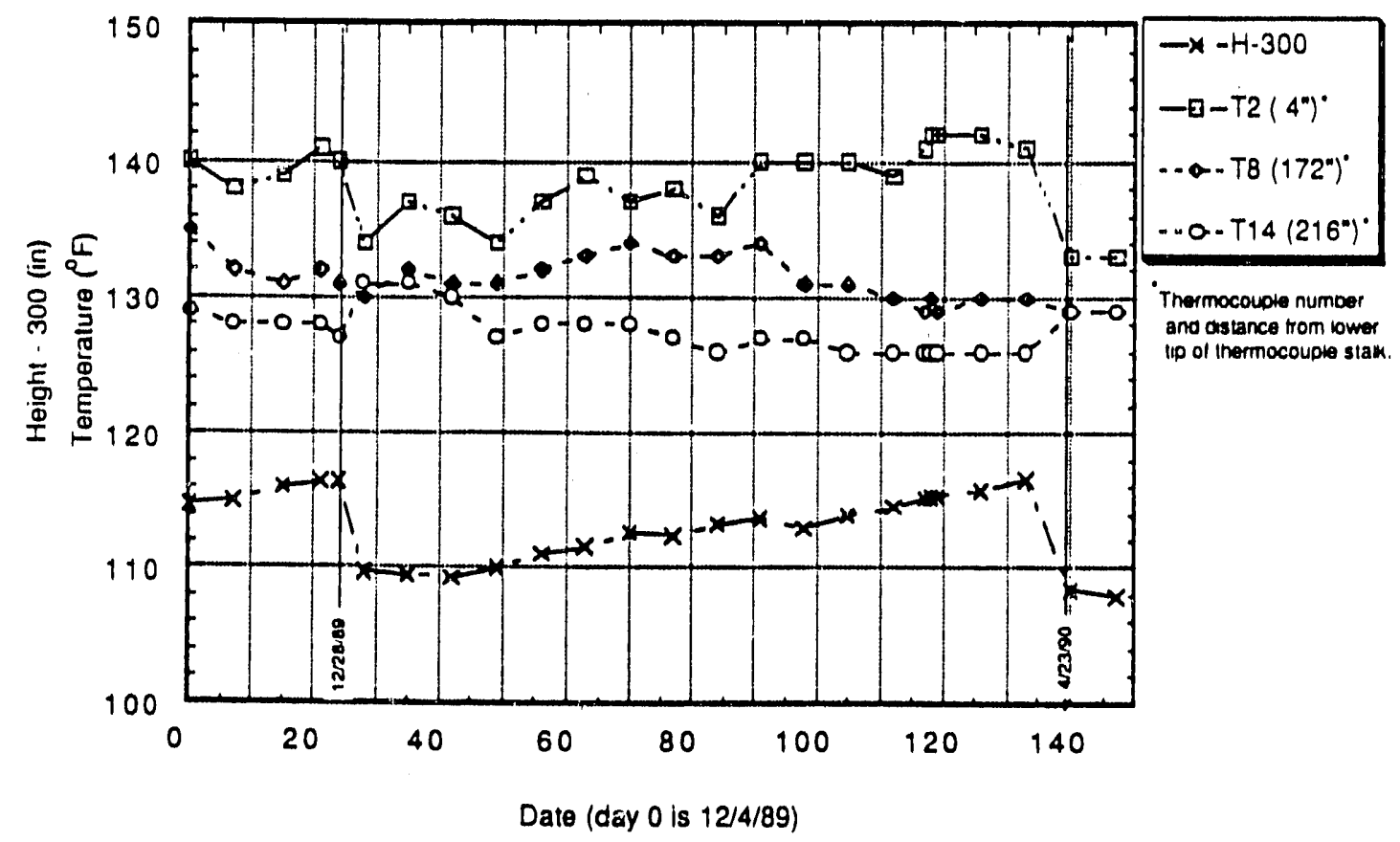

FIGURE 2.6. Relationship Between Temperatures at Thermocouples 2,8 , and 14 and the Level of the Tank Contents 
The relationship between the temperature changes at three locations in the tank and the level drops can also be seen in this figure.

The change in the liquid level was reported in a Rockwell internal letter to coincide with the increases in the tank head space pressure. Panjunen et a1. (1990) provided a detailed description of the relationship between tank pressurization, gas release, and liquid elevation level measured during the gas release event of April 19, 1990. Graphs showing the changes in these quantities during that gas release are reproduced in Figures 2.7 and 2.8 .

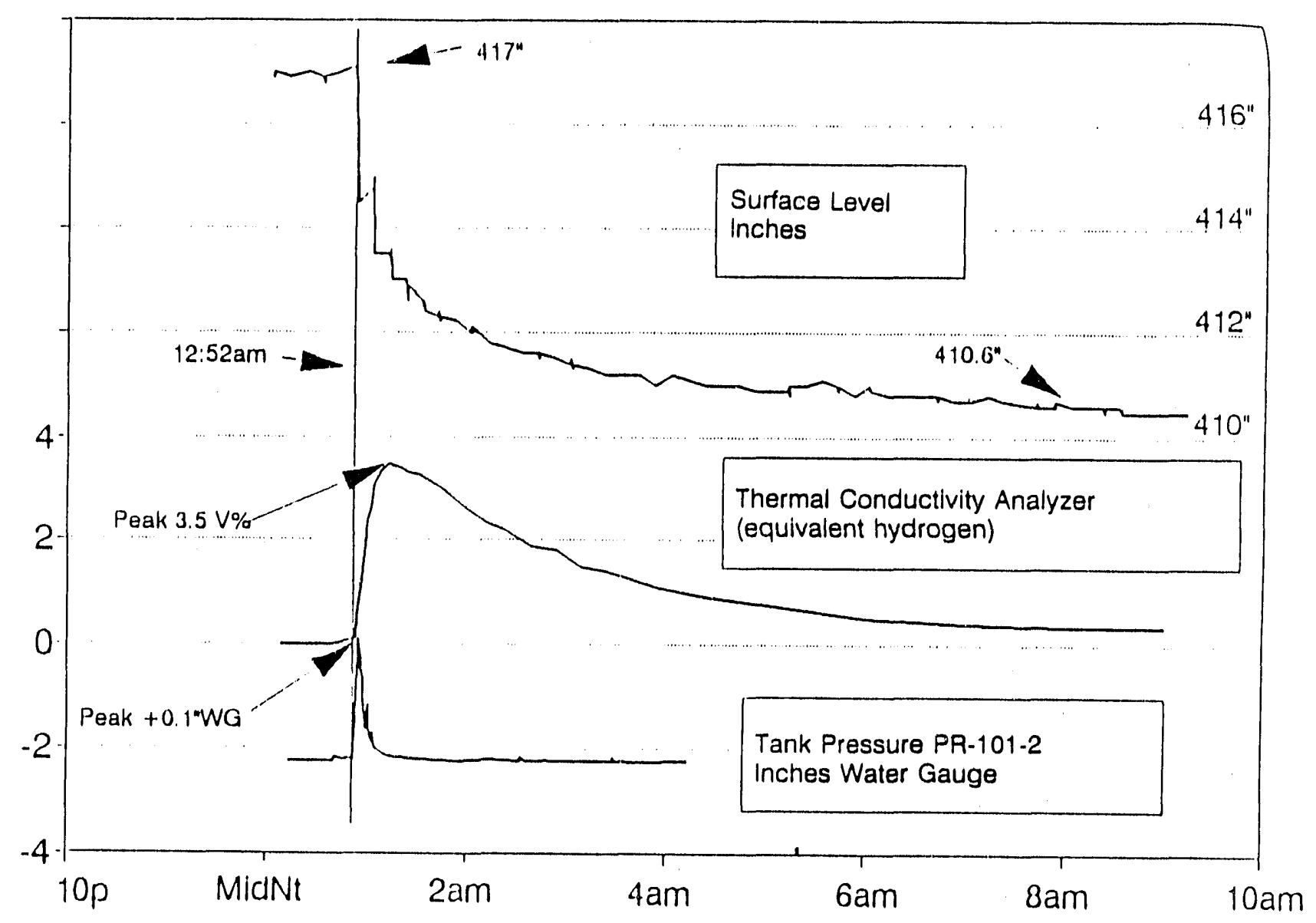

FIGURE 2.7. April 19, 1990, Event of Tank 101-SY, 12-h Plot 


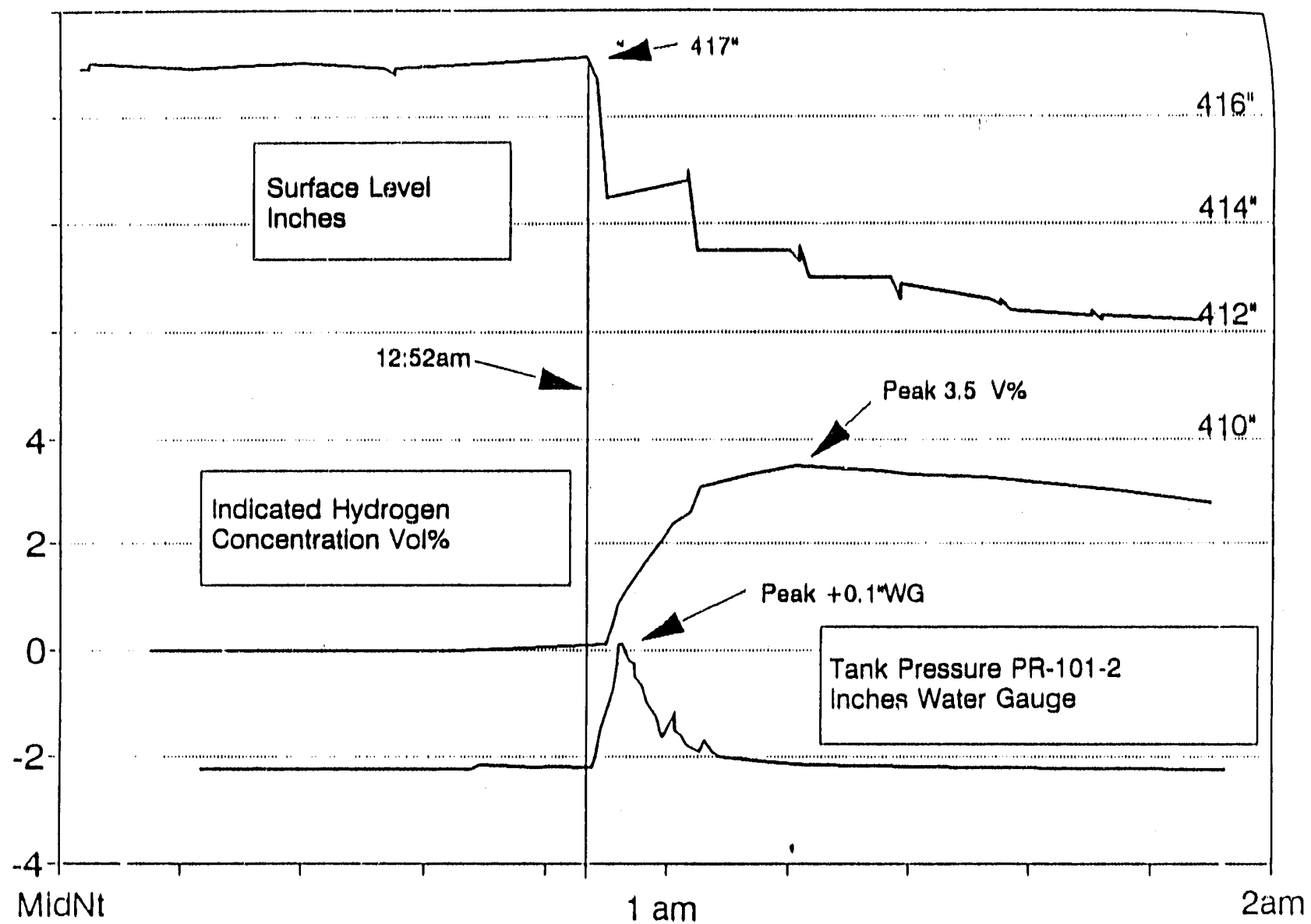

FIGURE 2.8. April 19, 1990, Event of Tank 101-SY, 2-h Plot

\subsection{TEMPERATURE CHANGES ASSOCIATED WITH THE LEVEL DROPS}

Currently, tank 101-SY is instrumented to allow temperature measurement at a number of locations in the tank. A temperature probe is fitted with 18 Type $J$ thermocouples located at distances of $4 \mathrm{in}$. to $460 \mathrm{in}$. from the probe tip. The location of the probe tip relative to the floor is not currently known. If the tip is assumed to be at the tank floor, the uppermost thermocouple (\#18) is in the head space and the second thermocouple from the top is in the head space when the tank level is below $412 \mathrm{in}$. Table 2.2 shows that the bent thermocouple probe places the lowest thermocouple at about $0.93 \mathrm{ft}$ from the tank bottom. Temperature readings taken using a hand-held readout device and the placement of these thermocouples on the probe were obtained from Dan Reynolds of Westinghouse Hanford. 
IABLE 2.2. Height Correction for Tilted Thermocouple Probe (for a thermocouple probe tilt angle of $8^{\circ}$ from vertical in Tank $101-S Y$ )

\begin{tabular}{lrrr}
$\begin{array}{c}\text { Thermo- } \\
\text { couple } \\
\text { Number }\end{array}$ & $\begin{array}{r}\text { As-Built } \\
\text { Height } \\
\text { (ft) }\end{array}$ & $\begin{array}{c}\text { sHeight } \\
\text { (ft) }\end{array}$ & $\begin{array}{r}\text { sheight } \\
\text { (in.) }\end{array}$ \\
\hline $101-1$ & 0.5 & 0.93 & 5.2 \\
$101-2$ & 2.5 & 2.91 & 5.0 \\
$101-3$ & 4.5 & 4.89 & 4.7 \\
$101-4$ & 6.5 & 6.87 & 4.5 \\
$101-5$ & 8.5 & 8.86 & 4.3 \\
$101-6$ & 10.5 & 10.84 & 4.0 \\
$101-7$ & 12.5 & 12.82 & 3.8 \\
$101-8$ & 14.5 & 14.8 & 3.6 \\
$101-9$ & 16.5 & 16.78 & 3.3 \\
$101-10$ & 18.5 & 18.76 & 3.1 \\
$101-11$ & 20.5 & 20.74 & 2.9 \\
$101-12$ & 22.5 & 22.72 & 2.6 \\
$101-13$ & 24.5 & 24.7 & 2.4 \\
$101-14$ & 26.5 & 26.68 & 2.2 \\
$101-15$ & 28.5 & 28.66 & 1.9 \\
$101-16$ & 30.5 & 30.64 & 1.7 \\
$101-17$ & 34.5 & 34.6 & 1.2 \\
$101-18$ & 38.5 & 38.56 & 0.8
\end{tabular}

Temperature measurements taken after the gas release event of April 19, 1990, are shown in Figures 2.9, 2.10, and 2.11. Some of these data have been plotted to show the evolution of the temperature profile at critical time periods between burps. It appears that adequate data on the tank temperatures are not available from the date of the burp. Apri1 19, 1990, until May 10, 1990. (a) The temperature $\log$ notes that readings were taken on the wrong switch. Figure 2.9 shows the evolution during the later weeks. Figure 2.10 shows the qualitative similarity between the profile recorded on April 16, 1990, and the profiles recorded on July 30, 1990, and December 28, 1989; all

(a) Temperature data for April 23, 1990, are reported in Preliminary Temperature Analysis. The temperature data that appear in that report are not consistent with the data that appear in the printout of a spreadsheet provided by $D$. Reynolds. The printout indicates that the thermocouple readout device was connected to the wrong switch, and that the appropriate temperature measurements were not performed. The discrepancy has not been investigated. However, the data in preliminary temperature analysis reports are currently considered suspect. 


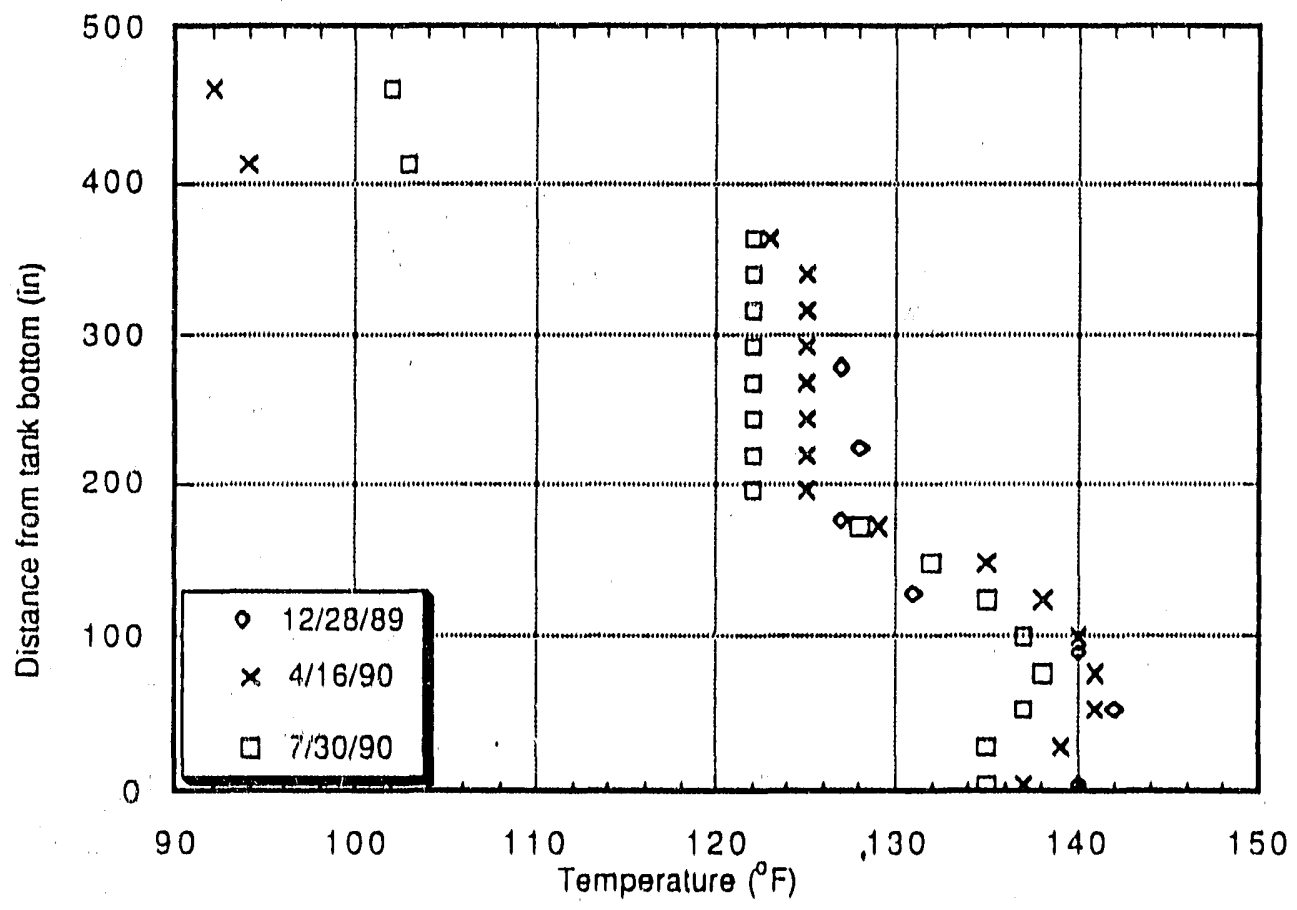

FIGURE 2.9. Comparison of Profiles Measured Prior to Level Drops

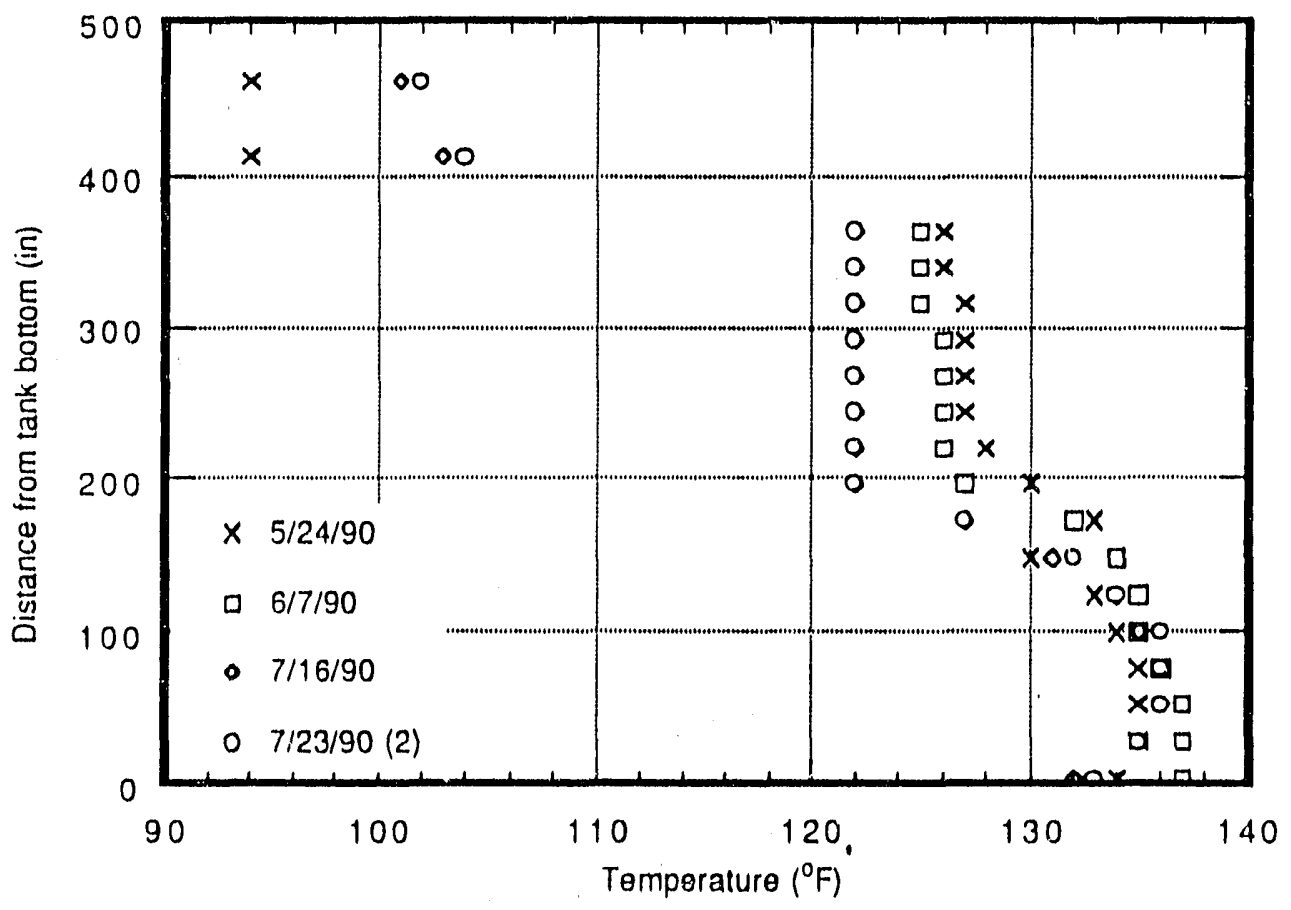

FIGURE 2.10. Temperature Evolution Between Tank Level Drops 


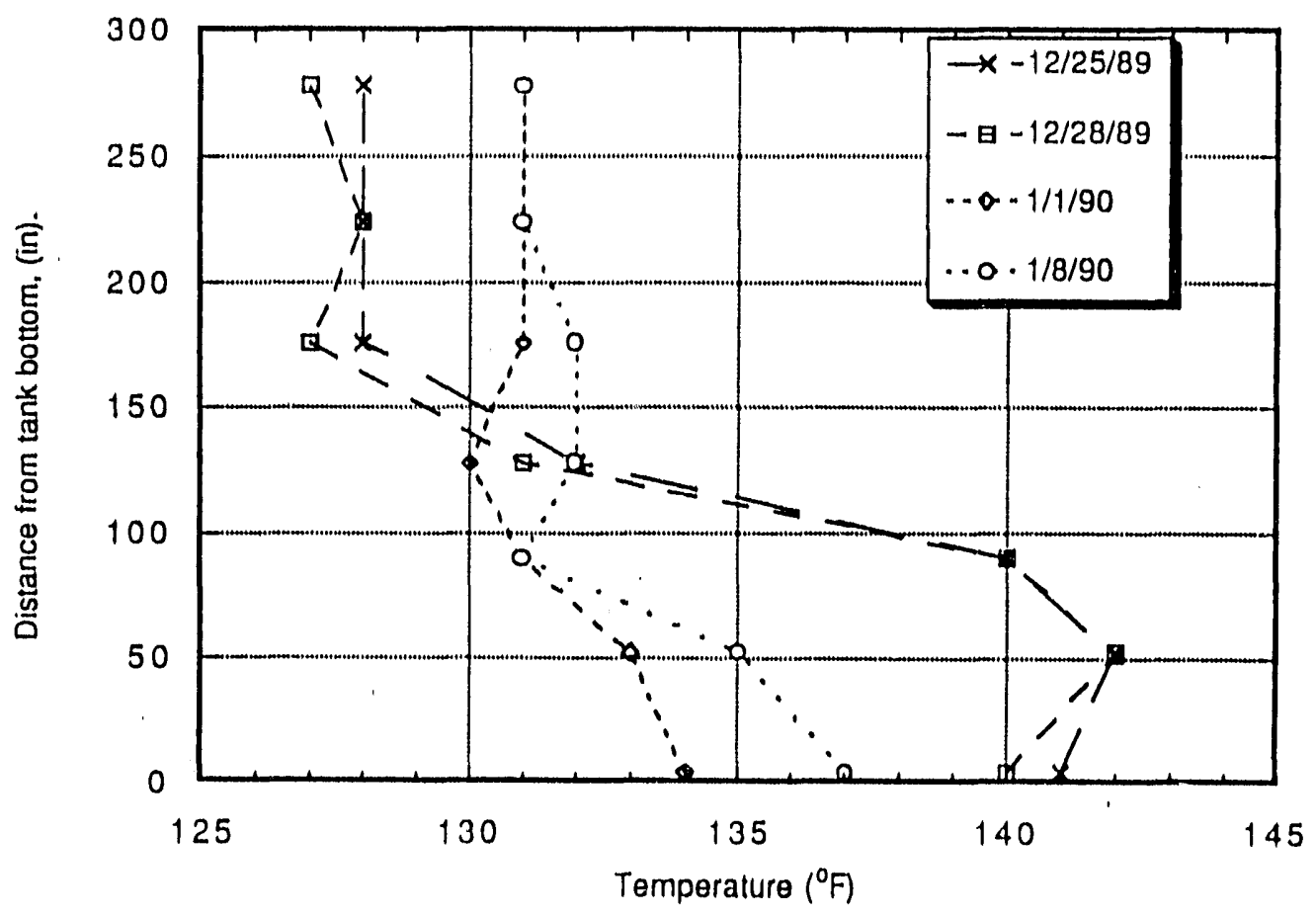

FIGURE 2.11. Temperatures Change for the Level Drop of December 1989

three profiles correspond to periods approximately one week prior to a level drop. Since no data were reported for the time period immediately after the April 19 gas release, the change in the temperature profile that occurrad during the previous gas release is shown in Figure 2.11. At that time fewer thermocouples were being read, so resolution of the profile shape is not as refined.

The changes in the temperature profiles shown here are typical of those recorded after a number of level drops including the ones that occurred before and after the level drop if August 8, 1990, and those that occurred after the level drop on October 24, 1990 . Details of the changes that occurred over a shorter period of time indicate that the temperature profile "inverts" during a gas release event. Immediately after the event, the temperature maximum exists at the top of the tank, and the minimum exists in the lower region of the tank. A temperature profile taken immediately after the gas release of october 24, 1990, is shown in Figure 2.12. This profile suggests an overturning of the tank contents during a gas release event. It is not clear whether 


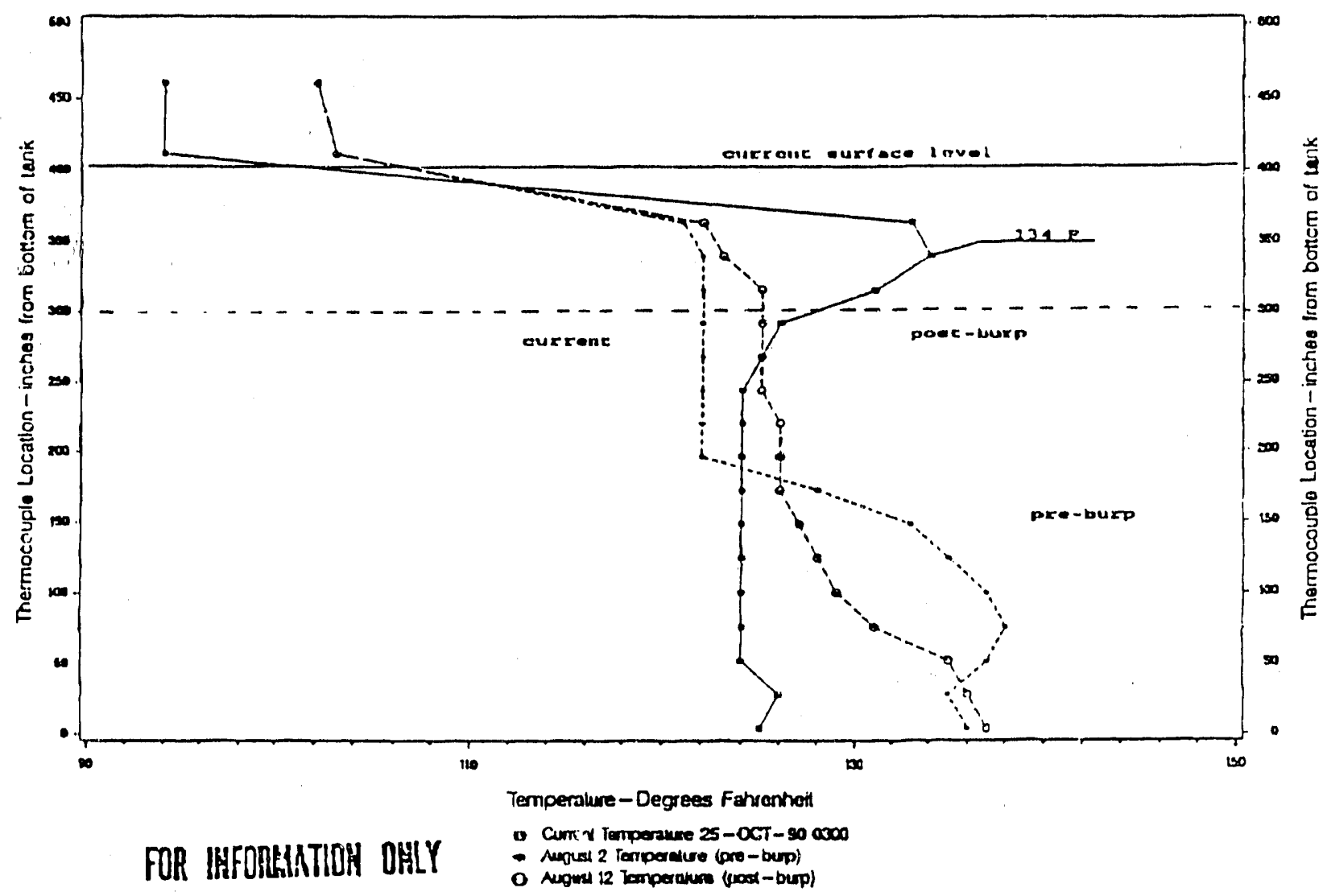

FIGURE 2.12. Current Profile and Pre- and Post-August 5, 1990, Burp Profile

similar temperature inversions have occurred in the past since temperature measurements were not reported at levels above $300 \mathrm{in}$. after the gas release of December 29, 1989. The temperature maximum occurs above this level, which is marked with a dashed line.

\subsection{PRESSURIZATION CHANGES IN THE TANK HEAD SPACE}

During normal operation, the tank head space is maintained at a negative gage pressure. The pressure has been observed to rise during level drops; occasionally this has resulted in positive gage pressures. The pressurization of the tank head space has been monitored since at least 1981 ; $^{(a)}$ a head

(a) D. R. Ellingson, Rockwell International, internal letter. 
space gage pressure of 1.8-in. $\mathrm{H}_{2} \mathrm{O}$ was observed on July 4,1983 , which was reported to be the first observation of a positive head space pressure. $A$ strip chart recording of the temperature transient was provided by Ellingson, but it was not legible on the copy obtained. The availability of better quality copies of these data has not been investigated. Tank head space pressures were monitored more recently during the gas release event of April 19, 1990, and are reported by Panjunen el al. (1990). The variation of the tank head space pressure based on this report is reproduced in Figures 2.7 and 2.8. The head space was pressurized suddenly, and then began to depressurize. Two further increases in pressure occurred during depressurization before the tank pressure returned to normal. This suggests that the total gas release consisted of three smaller releases.

Burke (1990) reports that the pattern of the head space pressurization during the release of Apri1 19, 1990, showed a number of spikes. However, during that gas release, the tank head space returned to normal before subsequent pressure spikes occurred. The pattern is similar to that which occurred in October in the sense that total release appears to have occurred in a number of smaller releases to the head space, but differs in magnitude.

\subsection{COMPOSITION AND VOLUME OF GAS RELEASES}

A detailed analysis of data gathered during the gas release that occurred on April 19, 1990, is reported by Panjunen et al. (1990). A gas sampling system was installed to allow analysis of gas composition in the duct. The hydrogen concentration in the ventilation duct during the gas release was monitored continuously using a conductivity analyzer. Grab samples were taken periodically and sampled using a mass spectrometer (CEC Model 21-130). Argon, oxygen, nitrogen, carbon dioxide, nitrous oxide, oxides of nitrogen, methane, and all constituents of normal air were detected during the gas release.

The measured gas composition and an estimate of the volume flow rate of the gas leaving the tank were used to determine the total volume of hydrogen released during a level drop. The composition of gas was also analyzed to determine the fraction of $\mathrm{N}_{2} \mathrm{O}$ contained in the gas; details of these analyses are reported by Panjunen et al. (1990). The volume flow rate of the gas leav- 
ing the tank was not measured during the level drop, but was assumed to equal that measured on April 24, 1990, five days after the burp. In reality, the gas flow rate might be expected to increase as a result of tank pressurization that occurs during the level drops; consequently, using the flow rate measured on April 24 to calculate the volume of slurry gases could cause the volume to be underpredicted. The estimated values of the volume and concentrations of $\mathrm{H}_{2}$ and $\mathrm{N}_{2} \mathrm{O}$ in the gas reported by Panjunen et al. are summarized in Table 2.3.

Earlier measurements indicated that the volume of hydrogen released in the level drop of July 16, 1985, was approximately $1000 \mathrm{ft}^{3}$, and that the total volume of gases released was $3800 \mathrm{ft}^{3}$. Hydrogen concentration was estimated to be $26 \%$ according to L. M. Sasaki in an internal letter. These values are smaller than those measured by Panjunen et al. (1990) for the level drop Apri1 24, 1990.

Analyses of later gas release events by Panjunen et al. are continuing, and results are being obtained as they become available.

\subsection{DURATION OF THE GAS RELEASE EVENTS}

There is a large degree of uncertainty in estimates of the duration of the gas releases. Panjunen et al. (1990) report that excess levels of hydrogen were detected by the Teledyne analyzer for a period of $132 \mathrm{~h}$ during the level drop of April 19, 1990. During this time, the tank level subsided $97 \%$ of the total level drop. The tank level continued to decrease for 10 days after the major gas release. However, the analyses of the resolution of the instrument suggest that the hydrogen concentration may have fallen to baseline levels in as little as $24 \mathrm{~h}$. In addition, the continuous concentration readings suggest that most of the hydrogen was released in a much shorter period of time.

TABLE 2.3. Summary of Gas Analysis Data (Panjunen et al. 1990)

Total Volume of Gas Released Concentration of $\mathrm{H}_{2}$ Concentration of $\mathrm{N}_{2}^{2} \mathrm{O}$

Estimated Duration of Release
Amount/Duration

6,000 to $11,000 \mathrm{ft}^{3}$
$30 \%$ to $57 \%$
$25 \%$ to $43 \%$
20 min to 10 days


Earlier measurements of the hydrogen concentration in the tank indicate that during the level drop of July 16, 1985, hydrogen was detected in the ventilation line for a period of $40 \mathrm{~h}$ after the initial tank pressurizatior per L. M. Sasaki (unpublished letter). This value falls between the maximum and minimum possible time periods during which excess hydrogen is reported to have been present in the ventilation lines during the April 19, 1990, level drop.

The time period during which hydrogen levels exceed baseline levels in the tank head space is expected to exceed the actual duration of the gas release. This is because the ventilation rate in the tank is finite. FATHOMS modeling of the tank response was performed and reported by Panjunen et al. (1990), which resulted in an estimate of a 20-min duration of the gas release event to match the tank pressurization data (including ventilation).

The difference between the duration of the gas release event based on FATHOMS modeling and that based on the measurement of tank level drops is over two orders of magnitude. Thus, the duration of the gas release event is not known with any degree of precision. It is possible that most of the gas is released quickly, and that small amounts of gas continue to be emitted over a period of time. For example, $6000 \mathrm{ft}^{3}$ of gas may be released in $20 \mathrm{~min}$, and the remaining $6500 \mathrm{ft}^{3}$ may be released over the course of 10 days. A release of this sort may be consistent with the FATHOMS modeling predictions and with the gas monitoring data.

It is expected that better estimates of the total gas release can be obtained if the actual ventilation rate in the vents from tank 101-SY is measured during a gas release. Equipment to measure the flow rate during a gas release has been installed, but no direct measurements of flow rates during a gas release are yet available.

\subsection{IANK CONTENTS: CHEMICAL COMPOSITION AND RHEOLOGICAL PROPERTIES}

Information on the chemical composition of the tank contents was obtained from two sources: an estimate of the tank chemical composition based 
on tank additions(a) and information based on chemical analysis of three samples taken from the tank. (b) Rheological information based on measurements of the effective viscosity of samples was reported by B. M. Mauss. (b)

\subsubsection{Chemical Composition of the Tank Contents}

An estimate of the chemical composition of both tanks 101-SY and 103-SY was reported by G. P. Simpson in an internal letter. Estimates for each tank were based on the best information available at the time. However, Simpson cautions that determination of the chemical composition and volume of the tank contents required accurate knowledge of both the composition and the volume of the material added to the tank; this information was not always available. Simpson reported five additions to tank 101-SY and two additions to tank 103-SY. A summary of Simpson's estimates of the tank compositions is included in Table 2.4. The estimated concentrations are based on an assumed value for the height of the tank contents given in the final column of Table 2.4.

TABLE 2.4. Estimated Composition of Tanks 101-SY and 103-SY Based on Additions Made Prior to September 1984

\begin{tabular}{|c|c|c|}
\hline Component & $\begin{array}{l}\text { Concentration } \\
\text { in Tank 101-SY } \\
\end{array}$ & $\begin{array}{l}\text { Concentration } \\
\text { (not current) } \\
\text { in Tank 103-Sy }\end{array}$ \\
\hline $\begin{array}{l}\mathrm{NaOH}(\mathrm{M}) \\
\mathrm{NaAlO}_{2}(\mathrm{M}) \\
\mathrm{NaNO}_{2} \text { (M) } \\
\mathrm{NaNO}_{3} \text { (M) } \\
\mathrm{Na}_{2} \mathrm{CO}_{3} \text { (M) } \\
\mathrm{Na}_{2} \mathrm{SO}_{4}(\mathrm{M}) \\
\mathrm{Na}_{2} \mathrm{PO}_{4}(\mathrm{M}) \\
\mathrm{Tota}_{4} \mathrm{Pu}(\mathrm{g}) \\
\text { Total } \mathrm{Sr}(\mathrm{mCi}) \\
\text { Total } \mathrm{Cs}(\mathrm{mCi}) \\
\text { Total organic carbon (TOC) (g/L) } \\
\text { Total } \mathrm{H}_{2} \mathrm{O} \text { (gal) } \\
\text { Estimate of original height (in.) } \\
\text { Total height reported in } 1984 \text { (in.) }\end{array}$ & $\begin{array}{l}3.22 \\
1.90 \\
3.28 \\
4.23 \\
0.62 \\
0.12 \\
0.19 \\
713 \\
2.187 \times 10^{11} \\
3.102 \times 10^{12} \\
26.24 \\
594,600 \\
386.85 \\
408\end{array}$ & $\begin{array}{l}29.48 \\
266,130 \\
178.3 \\
190\end{array}$ \\
\hline
\end{tabular}

(a) G. P. Simpson, Rockwell International, internal letter.

(b) B. M. Mauss, Rockwell International, internal letter. 
An estimate of the unexpanded tank height is included in Table 2.4. This estimate is based on the records of the volume additions to tank 101-SY. No level measurements giving the tank contents at this low a level have been reported. Consequently, it is not clear whether the true unexpanded height is $387 \mathrm{in}$. as indicated by Simpson or if the unexpanded height is somewhat larger. The lowest tank level measured since 1980 is 403 in.; this occurred in 1984 and can be seen in Figure 2.5.

It is worth noting the similarities and differences between the contents of tank 103-SY and tank 101-SY since both tanks exhibit growth and have exhibited level drops. Currently, however, the level drops in tank 103-SY are only 1 to 2 in. in magnitude. In 1984, tank 103-SY appeared to exhibit a larger fractional amount of growth (6.5\%) than tank $101-S Y(5.5 \%)$. In addition, tank 103-SY was reported by L. M. Sasaki (internal letter) to have exhibited as much as $17 \%$ growth prior to subsiding. If true, this suggests that tank 103-SY was at one time exhibiting larger level changes than observed in tank 101-SY, where the maximum growth observed was approximately $9 \%$ (37 in.) of the initial fill height of $387 \mathrm{in}$.

The contents of tank 103-SY estimated in 1984 are also shown in Table 2.4. It is clear that the two tanks have similar concentrations of sodium compounds, organics, and water. However, tank 101-SY contains plutonium, strontium, and cesium, which appear to be absent from tank 103-SY. The current contents of tank 103-SY should differ from those noted in Table 2.4 since there have been numerous additions since that time.

Chemical analyses were performed on three samples that were removed from tank 101-SY using the "dip" or "bottle on string" method. The exact method of obtaining samples was not reported by Mauss (internal letter). The three samples are referred to as "top," "middle," and "bottom"; the tank elevation at which these samples were taken was not provided by Mauss. Since collection using the dip method requires that fluid flow into the bottle, it is unlikely that the top sample corresponds to pure crust.

The chemical composition of the slurry determined by chemical analyses of the three samples was reported by Mauss. Top and middle samples were separated using vacuum filtration; the composition of both the slurry and the 
liquid were reported for these two samples. The bottom sample could not be separated, thus only the slurry composition was reported for this sample. The compositions of the top, middle, and bottom slurries as reported by Mauss are shown in Table 2.5. Some probable errors in the reported numbers are noted in the table footnotes.

All samples contained significant quantities of undissolved solids. The volume concentrations of solids in the three samples were $18.3 \%, 27.8 \%$, and approximately $60 \%$ for the top, middle, and bottom samples, respectively. The reliability of the 60 vol\% solids for the bottom sample may be questionable, particularly since $47 \%$ of the sample is reported to be 1 iquid. However, a very high solids concentration is consistent with a verbal description of the sample provided by Mauss.

The chemical analyses indicate the presence of components that were not included in the estimate of tank contents as reported by Simpson. In addition, the analyses indicate that the bottom sample contains the greatest concentration of most of the radioactive components, although this is not true for all radioactive components.

The Mauss letter states that carbon-14 and iodine-129 were not detected in the sample. This was not considered credible, and an addendum to the report was to be published after the analysis technique was revised. This addendum has not been located.

The samples were also analyzed by polarized light microscopy, by $x$-ray fluorescence (XRF), and by $x$-ray diffraction. The results of these tests were described by Mauss. $X$-ray analysis indicated that the bottom sample contained corrensite or some similar clay-like material. This may have some effect on the mixture rheology, since the addition of clay to mixtures can affect the rheological behavior of slurries significantly. The addition of small amounts of clay can lead to non-Newtonian behavior (Weyl and Ormsby 1960). Behaviors typical of clay mixtures and slurries containing sufficient quantities of clay include gel-sol transitions (i.e, thixotropy) and yield pseudoplastic behavior behavior. The existence of either a yield stress, as seen in a pseudoplastic fluid, or a shear strength exhibited by thixotropic materials could enhance 
TABLE 2.5. Composition of Tank 101-SY Reported by Mauss (interrial letter)

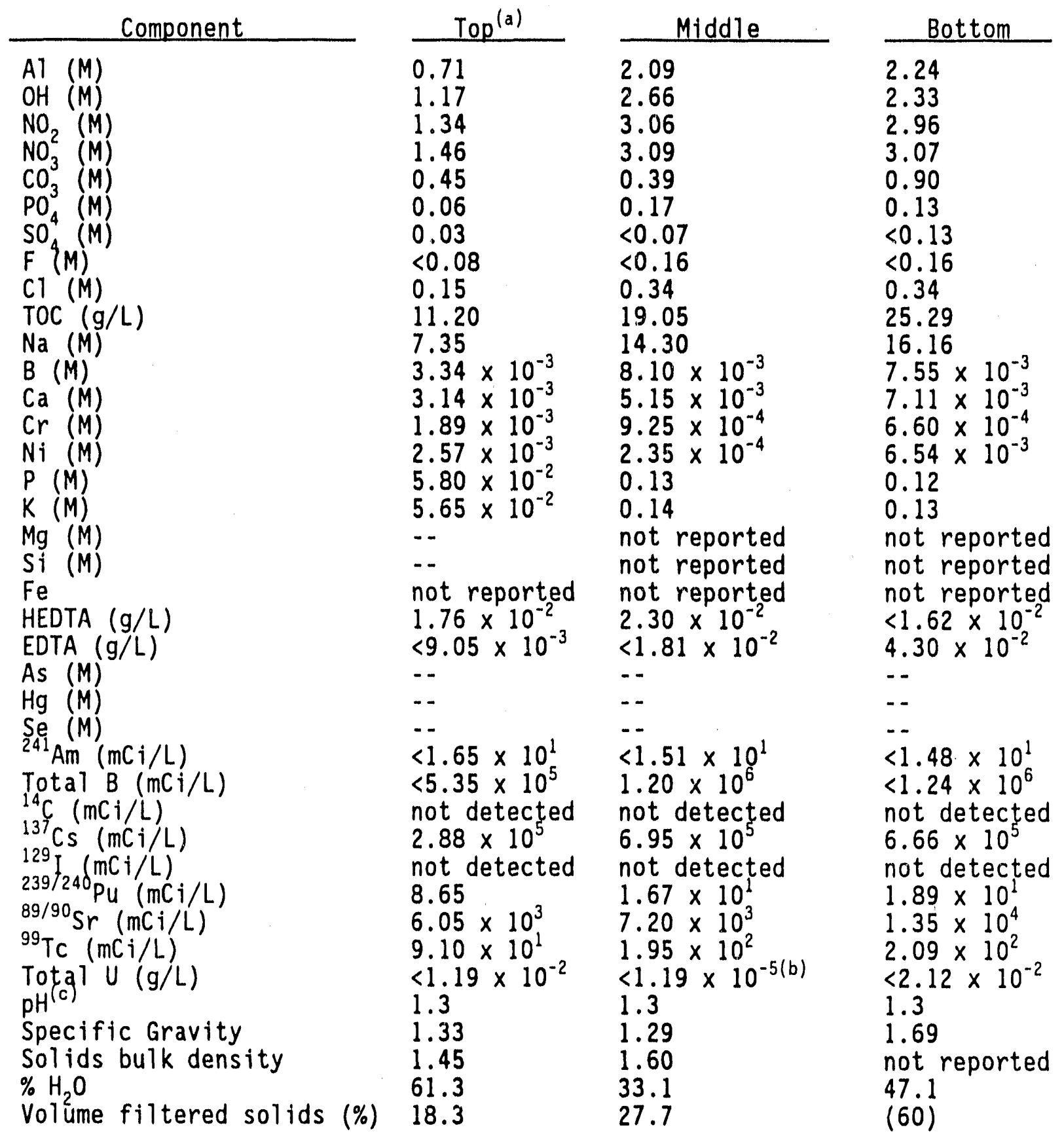

(a) It is likely that there is a factor of two dilution error in the top sample. The concentrations should be multiplied by two.

(b) Based on the values in the top and bottom sample, the exponent here seems unlikely. The reported quantity of uranium in the sample should be verified against the analysis records.

(c) The $\mathrm{pH}$ value of 1.3 reported for all samples is not credible. It has been suggested that this is a misprint and should read 13 . 
the ability of the slurry to trap gases within it. The observed rheological behavior of the slurry is discussed in Section 2.6.2.

Mauss also provided qualitative descriptions of the three samples:

The top sample was a slurry with approximately $45 \%$ settled solids which were dark gray and fine (clay-like) in appearance. The supernatant was clear yellow. The middle sample was a murky, brownish-gray slurry. When centrifuged the supernatant turned greenish-yellow and the solids were fine and brownish gray. The bottom sample was very thick (sludgy), with no settled solids. The sample was a green-gray color.

\subsubsection{Rheology of the Tank Contents}

The three samples analyzed by Mauss were also analyzed to obtain the sample viscosity and specific gravity. The reported values of the fluid viscosity are shown in Table 2.6. The measurements were obtained using a Haake viscometer. Unfortunately, the complete rheograms on which the reported data are based were not included by Mauss in the report; instead the effective viscosity data are reported at four strain rates. The availability of the complete rheograms has not been investigated; the figures included here rely exclusively on the information reported by Mauss.

The reported viscosity values were used to obtain the stress-strain curves, Figures 2.13,2.14, and 2.15. The stress-strain curves suggest that the bottom sample may be non-Newtonian; the intercept for the stress-strain curve based on the increasing strain rate data indicates that the material has

TABLE 2.6. Effective Viscosity of Undiluted Slurry Samples from Tank 101-SY (as reported by Mauss [internal letter]: Viscosity values in $\left.C P\left[10^{-3} \mathrm{~Pa}-\mathrm{s}\right]\right)$

\begin{tabular}{|c|c|c|c|c|c|c|}
\hline \multirow{2}{*}{$\begin{array}{c}\text { Shear Rate, } \\
\mathrm{s}^{-1} \\
\end{array}$} & \multicolumn{2}{|c|}{ Top } & \multicolumn{2}{|c|}{ Middle } & \multicolumn{2}{|c|}{ Bottom } \\
\hline & $60^{\circ} \mathrm{C}$ & $50^{\circ} \mathrm{C}$ & $60^{\circ} \mathrm{C}$ & $50^{\circ} \mathrm{C}$ & $60^{\circ} \mathrm{C}$ & $50^{\circ} \mathrm{C}$ \\
\hline $\begin{array}{r}346 . \\
692 . \\
1385 \\
1385 \\
692 . \\
346 . \\
173 .\end{array}$ & $\begin{array}{r}20.5 \\
7.7 \\
7.2 \\
8.0 \\
6.9 \\
5.9 \\
6.2 \\
21.6\end{array}$ & $\begin{array}{r}20.5 \\
8.2 \\
8.5 \\
10.0 \\
9.8 \\
7.7 \\
7.2 \\
16.4\end{array}$ & & & $\begin{array}{l}86.3 \\
74.5 \\
61.9 \\
52.0 \\
51.0 \\
55.5 \\
63.7 \\
74.0\end{array}$ & $\begin{array}{l}7 \\
7 \\
2 \\
1 \\
2 \\
3 \\
0\end{array}$ \\
\hline
\end{tabular}




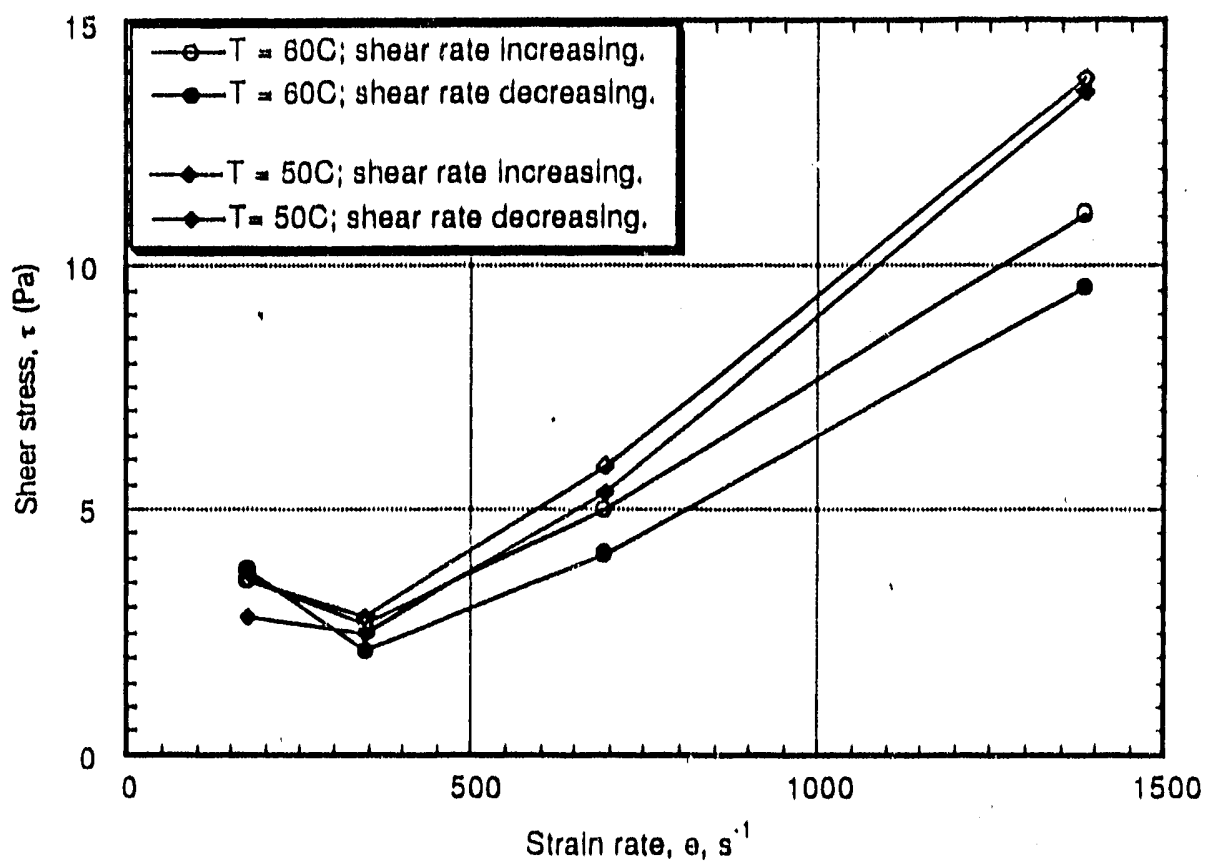

FIGURE 2.13. Stress Versus Strain Curves for the Contents of Tank 101-SY, Top Sample

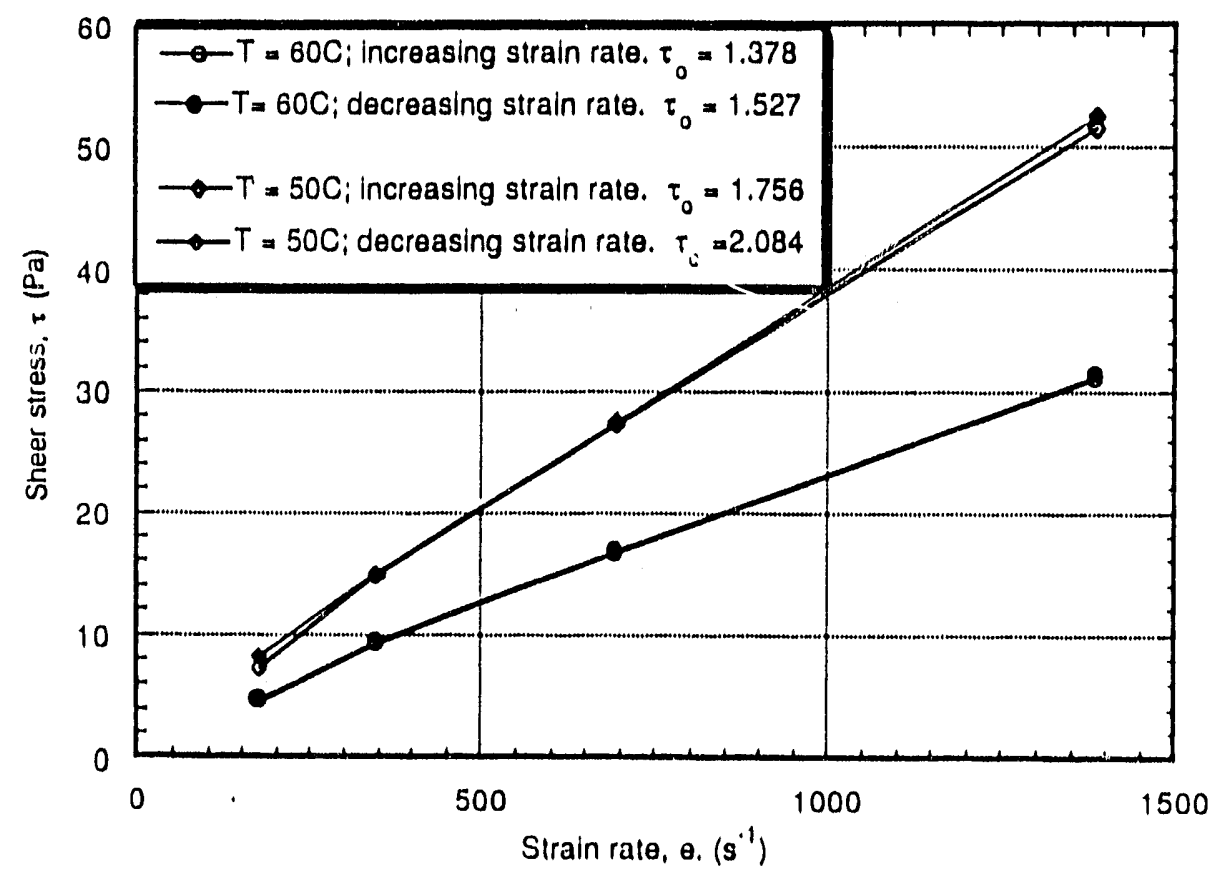

FIGURE 2.14. Stress Versus Strain Curves for the Contents of Tank 101-SY, Middle Sample 


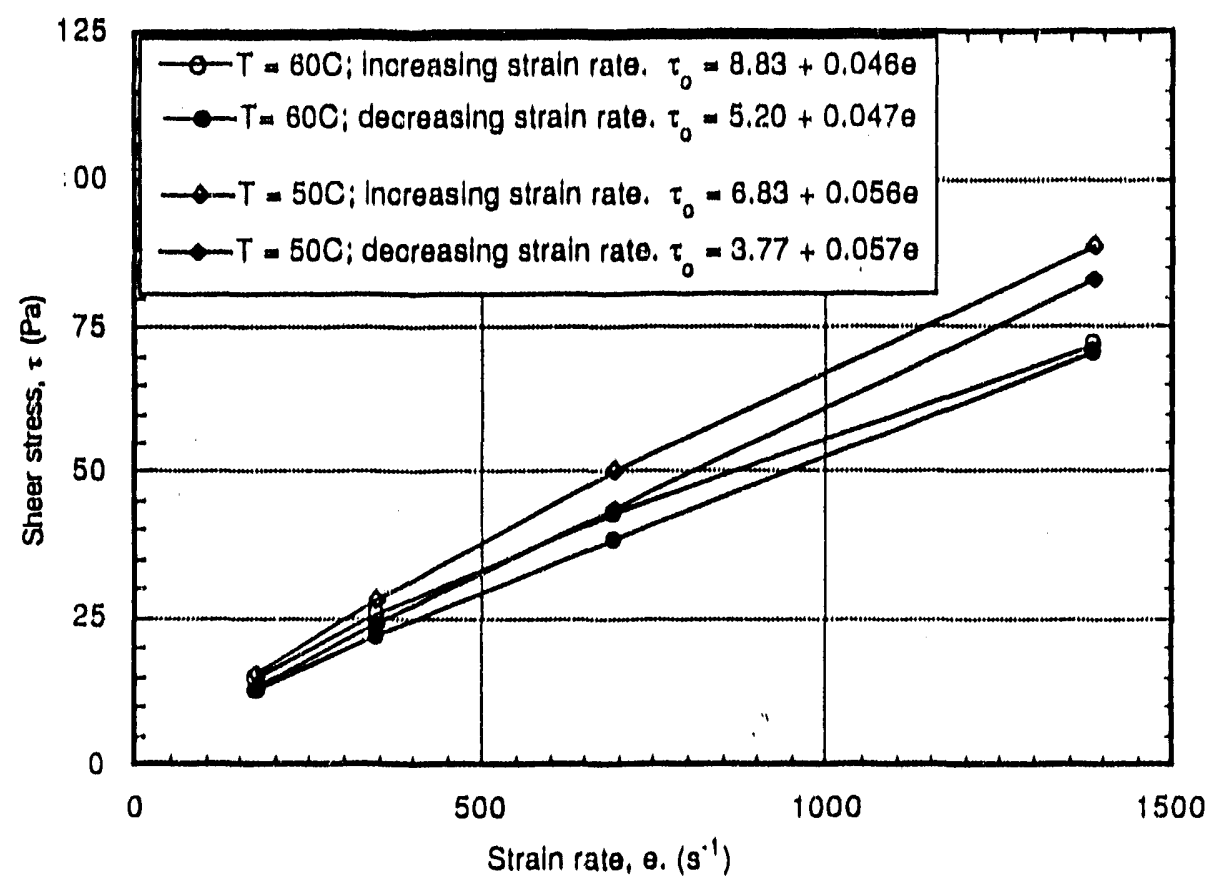

EIGURE 2.15. Stress Versus Strain Curves for the Contents of Tank $101-S Y$, Bottom Sample

a yield stress of approximately $8.8 \mathrm{~Pa}$. Much more detailed and accurate rheological information providing both the yield stress and consistency index could be obtained from the complete rheograms if they are in the archive material.

Non-Newtonian behavior is not unique to tank 101-SY; it has also been observed for the contents of tank 103-SY. The yield stress of the contents of tank 103-SY was determined to be approximately $\tau_{y}=1.8 \times 10^{1} \mathrm{~Pa}\left(1.8 \times 10^{2}\right.$ dyne $/ \mathrm{cm}^{2}$ ) according to $W$. 0 . Heath in an internal letter. This is approximately twice the value of the yield stress obtained by applying a curve fit to the data for the bottom sample in tank 101-SY reported by Mauss.

The viscosity measurements reported by Mauss appear to have been performed at increasing and then decreasing rate of strain, as would normaliy be done using a Haake viscometer. The measurements indicate that the viscosity at increasing strain rate is consistently higher than at decreasing strain rate, which suggests that the fluid may be thixotropic, although this is not certain. Direct observations of gelling behavior and thixotropy have been 
reported by $W$. 0 . Heath for the contents of tank 103-SY. In addition, dilatant behavior has been observed by J. L. Starr (internal letter) in synthetic evaporator slurries that contain EDTA (ethylene diamine tetracedic acid) and HEDTA (hydroxyethyl-ethylene diamine triacedic acid); both of these organics are contained in the slurry in tank 101-SY. Thus, thixotropic behavior suggested by the viscosity data also appears to be likely based on the behaviors observed in similar tank wastes and observed in synthetic slurries.

Thixotropic materials often exhibit shear strength behavior prior to the onset of motion. The shear strength of a material is a material's resistance to strain exhibited when the muterial is in a "solid" state. Measurement requires that the material be allowed to sit undisturbed for a period of time during which the material solidifies. When a constant strain rate is applied to the solidified sample, the shear stress in a material exhibiting a shear strength will vary, as shown in Figure 2.16. The material will resist deformation until a maximum stress corresponding to the shear strength, $\tau_{s}$, is reached. The material will then begin to flow like a fluid and exhibit a

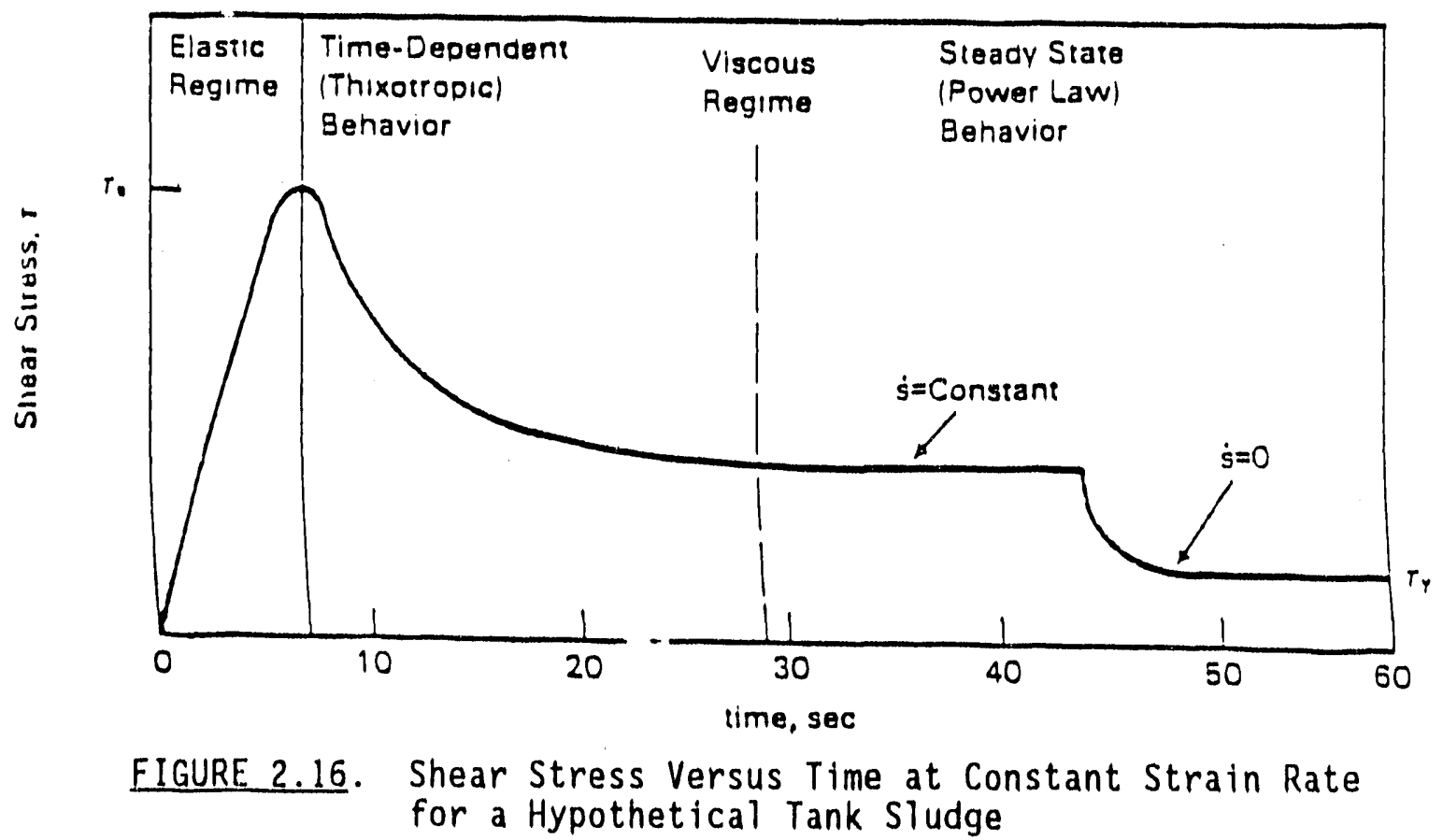


time-dependent stress even though the applied strain rate is consiant. Finally, the stress experienced at the applied strain rate will fall to some asymptotic level.

Once fluid-like behavior has been achieved, it is possible for the fluid to have efther Newtonian or non-Newtonian rheology. Figure 2.16 shows behavfor typical of a non-Newtonian fluid, where it is possible to reduce the strain rate to zero and still detect a yield stress, $\tau_{y}$, in the fluid. This yield stress is the stress that must be overcome to deform the fluid when it is in its fluid state. A thixotropic fluid with no yield stress would show a shear stress of zero when the fluid is not being deformed. The shear strength for a thixotropic material is often much greater than the "yield" stress exhlbited when flowing as a fluid. Shear strength of the contents of tank 101-SY has not been reported. The reported shear strength of the sample from tank 103-SY described above was approximately $\sigma_{s}=3.2 \times 10^{3} \mathrm{~N} / \mathrm{m}^{2}$, (a) which is two orders of magnitude larger than the yield strength observed once the fluid motion begins. It is possible that the material in tank 101-SY also has a large shear strength, but there are no data on shear strength for the contents of this tank.

Possible thixotropic and dilatant or gelling behavior of the material in tank 101-SY is important in terms of some of the proposed gas bubble release mechanisms since a high shear strength would suggest that extremely large bubbles could be trapped by the slurry. The possible existence of either a yield stress or a shear strength for the contents of tank 101-SY must be explored, since either could play an important role in trapping gases. Either a finite yield stress or shear strength could significantly enhance the ability of the slurry to trap gas.

\subsection{DATA ON SLURRY GROWTH STUDIES USING SIMULATED SLURRIES}

The phenomenon of slurry growth was studied extensively by the tank farm operators. It was assumed that organics present in the tank reacted and produced gas; growth occurred when the gas was trapped by the slurry.

(a) W. 0. Heath, Pacific Northwest Laboratories, internal letter. 
Nonradioactive simulated slurries were prepared using varying concentrations of organics. Initial studies focused on the rate of gas generation in the synthetic slurries; the relationship between gas generation and slurry growth was not discussed. In later experiments, the effect of slurry viscosity on the slurry growth was studied.

Factors found to affect the gas generation rate include the chemtcal composition and temperature of the simulant. A correlation based on the simulant temperature and chemical composition was developed by Jansky (1985). Equations predicting the rate of gas generation by chemical means have also been proposed by Delegard (1980). However, the design of the experiments on which these equations are based was criticized by Rees in an internal letter, who states that the correlations can only be interpreted as providing a qualitative description of the effect of concentration and temperature on the slurry gas growth rate. This is because the actual temperatures and concentrations at which the slurry gas generation rates were measured fall outside the range that occurs in the tank. Reynolds (internal letter) made a similar criticism of these experiments and suggested that later experiments be performed near tank temperatures and concentrations. Both Reynolds and Rees recommend that the equations provided by Delegard and Jansky not be used to obtain quantitative estimates of the gas generation rate in the slurry.

Despite the extensive number of slurry growth studies, it appears that a reliable correlation for the rate of gas production due to chemical reactions in tank wastes does not exist. In addition, it is not clear how any such equation should be applied since it was developed using a homogeneous simulant, while the slurry in tank 101-SY appears to be stratified. This suggests that more gas may be generated in regions with high concentrations of the reactive chemicals and less in other regions, and that prediction of the rates would require a more detailed knowledge of the distribution of the chemicals in the tank than is currently available.

In addition, the correlations proposed by Jansky (1985) and Delegard (1980) predict gas generation that is due solely to chemical reaction of organic products in the simulants. No experimental measurements of radiolysis have been conducted. Predictive analyses suggest that the gas generation rate 
due to radiolysis may be of the same magnitude as that predicted on the basis of the chemical composition of the slurry according to Gough (in a memo). Gough also notes that oxygen produced by radiolysis may inhibit the chemical reactions in the tank. If so, experiments such as those performed by Jansky would not produce reliable predictions of the gas generation rate since they were not carried out using radioactive wastes.

It is likely that the mechanism for gas generation is important in determining the gas generation rate and gas composition. However, regardless of the gas generation mechanism, slurry growth cannot occur in the absence of a mechanism for gas entrapment. The rate at which gas is generated may not affect the total quantity of gas trapped since it is likely that this would be more strongly affected by the shear strength, yield stress, and viscosity of the slurry, or by the strength of the crust. Consequently, the gas generation rate may not govern the ultimate level of slurry growth but could affect the time period between tank level drops.

Later experiments focused on the role of slurry viscosity on slurry growth. Jansky (1982) reports that simulants were boiled down to concentrations that produced slurries with viscosities of $100 \mathrm{cP}, 500 \mathrm{cP}$, and $1000 \mathrm{cP}$. The most viscous slurry was found to trap the largest quantities of gas and to expand as much as $94 \%$ in volume. Jansky describes these highly expanded slurries as being foamy and resembling bread dough in consistency. Cyclic rise and fall behaviors on the part of these simulants have been described by Jansky (1982). In conversation, Jansky indicated that these simulants did not collapse fully and that they retained their foamy nature throughout the rise and fall process.

It is worth noting that the partial collapse behavior seen in Jansky's experiment may be similar to the rise and fall behavior observed in tank 101-SY. The level of the tank conients does not appear to collapse back to the initial fill height after each drop. If the estimate of the initial fill height provided by Simpson (internal letter) is accurate, the contents never fall to the original level.

There are also a number of important differences between the cyclic behavior observed in the simulated slurries and those occurring in 
tank 101-SY. First, the magnitude of cyclic level drops observed by Jansky were small; for example, the rise and fall seen in the 500-cP simulant was less than $5 \%$ of the total growth exhibited. In addition, the total growth observed in the simulants was as large as $94 \%$, which is much greater proportionally than observed in tank 101-SY. Thus, the underlying physical mechanism of the cyclic behavior observed in the foam-like simulants may differ from the one causing cyclic behavior in the tank. Nevertheless, the cyclic growth behavior observed in Jansky's synthetic slurries appears to be the only report of any form of cyclic growth observed in laboratory studies.

The experiments performed by Jansky appear to have assumed that the synthetic slurries were Newtonian since the slurries were characterized using a single value for the viscosity. (a) This viscosity was measured using a spindle viscometer. Unfortunately, it is not possible to determine (based on the acquired references) whether the synthetic slurries used by Jansky behaved as Newtonian fluids or if they may have had a yield stress.

Another factor that has been reported to be related to the degree of slurry growth is the tank volume. Simpson (1984a) reports that the magnitude of slurry growth observed in tanks and in bench-scale experiments using simulants is inversely related to tank volume. Large amounts of slurry growth are observed to occur in 1-L flasks, while lower slurry growth is observed in larger test vessels and in actual tanks. A physical reason for this was not suggested. In contrast, Janiky and Horton (internal letter) report that tank volume affects the growth rate of simulated slurries but not the maximum growth.

The data on waste volume, tank height, and slurry growth from Simpson are replotted and shown in Figures 2.17 and 2.18. Exponential curve fits to the data suggest that slurry growth is more closely tied to the tank's height than its volume. This may suggest that large hydrostatic pressures reduce the volume of gas that is trapped by the slurries.

(a) It should be noted that slurries such as those in tank 101-sy are often found to exhibit non-Newtonian rheological properties; they frequently also exhibit pseudoplastic behavior. 


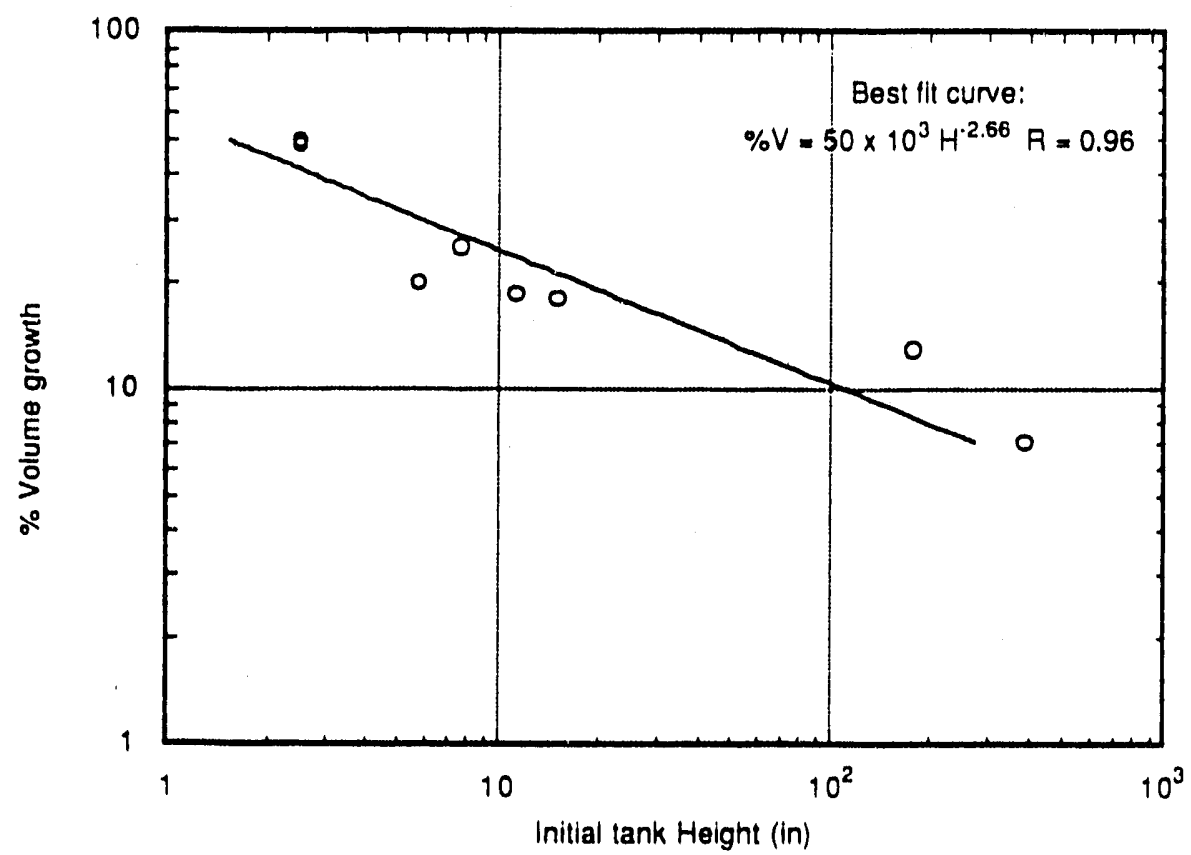

FIGURE 2.17. Observed Relationship Between Waste Growth and the Initial Height of the Tank Contents

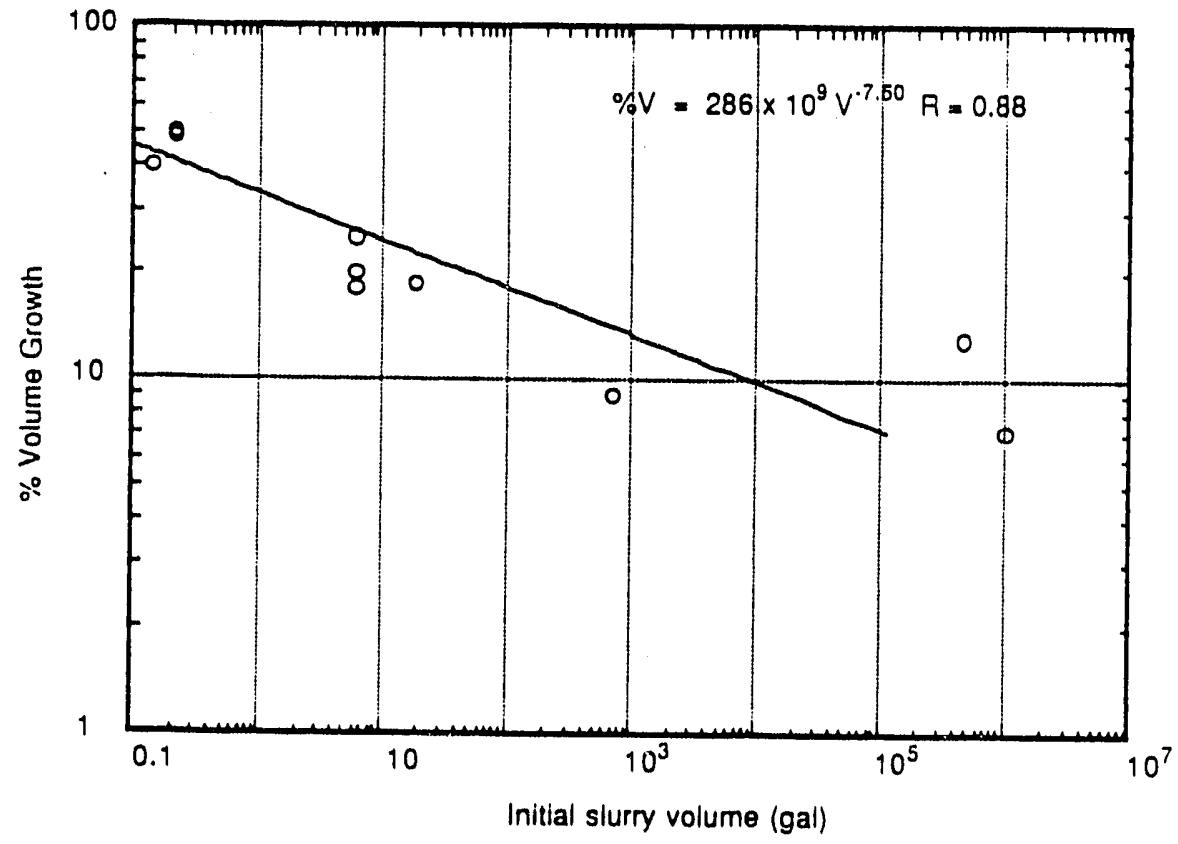

FIGURE 2.18. Observed Relationship Between Waste Growth and Tank Volume 
It is not clear whether the observed relationship has any significarice. The composition of the simulated wastes that grew in the small vessels differed from the composition of the real wastes in the tank. However, future laboratory experiments performed to study gas entrapment and release should be performed at a minimum of two scales in order to ensure either that the physical phenomena observed are unaffected by scale or to determine the effect of scale on the phenomena.

\subsection{COMPARISON OF THE RECENT GAS-RELEASE EVENTS}

Table 2.7 shows data obtained for several recent gas releases from tank 101-SY; the discussion in the following sections makes some comparisons of these data. The "consolidated level" is the level of the waste after the event.

\subsubsection{Special Features of the Gas Releases}

Approximately $7000 \mathrm{ft}^{3}$ of gas was released in April 1990 in about $40 \mathrm{~min}$; a slow release followed over the next few days. Even though a similar volume of gas was released in October, it occurred within the first 3 to $4 \mathrm{~min}$ and then over a few hours. The August release was unlike either of the other two events since only half the volume $\left(-3400 \mathrm{ft}^{3}\right)$ was released in a series of 15 to 20 short spurts over a period of $3 \mathrm{~h}$.

\subsubsection{Flow Rate Variation}

The steady-state vent flow has been continuously increased since the April event, in an effort to reduce the peak hydrogen concentration in the tank. This air flow variation may also affect the rate of evaporation from the surface of tank 101-SY.

\subsubsection{Pressure Variation}

The pressure rises were considerably smaller in the April and August events, but a larger pressure spike occurred in the 0stober event. It is not clear what the final maximum pressure rise in the header was, but it seemed to fall to the steady-state value in about 12 to 14 min after the start of the event. The higher pressure buildup and a larger gas release in a shorter time-span in October may be the results of the smaller release in August. 
TABLE 2.7. Data on Recent Gas Releases, Tank 101-SY

Time of event

Time between events, weeks

Max. crust level (in.)

Consolidated level (in.)

Avg volume change/day between events ( $\left.\mathrm{ft}^{3} / \mathrm{day}\right)$

Pressure at peak of event (in. water gage)

flow rate during

- steady-state (cfm)

- Gas-release rate (cfm)

- Total volume of gases $\left(\mathrm{ft}^{3}\right)$

Based on crust fall (in.)

Based on FATHOMS model

Gas volume ratio

Percentage of $\mathrm{H}_{2}$ in the release

Measured

Predicted

\begin{tabular}{|c|c|c|c|}
\hline $12 / 23 / 86$ & $4 / 19 / 90$ & $8 / 5 / 90$ & $10 / 24 / 90$ \\
\hline-- & $12: 52 \mathrm{am}$ & $05: 30 \mathrm{am}$ & $06: 05 \mathrm{am}$ \\
\hline 7 & 8 & 15 & 11 \\
\hline 411.2 & 417 & 416 & 412.2 \\
\hline 407.5 & 410.6 & 410.6 & 404.3 \\
\hline
\end{tabular}

27.8

42.1

18.9

37.8

$+0.1$

$-1.95$

$+2$

370

417

611

370

417

$>1200$

3400

1988

3387

(9.3)

(5.4)

( 9.2)

$-7000 \mathrm{ft}^{3}$

in $20 \mathrm{~min}$

$-3400 \mathrm{ft}^{3}$

in $3 \mathrm{~h}$

$-7000 \mathrm{ft}^{3}$

2.1

1.7

2.3

3.5

1.2

4.8

3.9

1.3

5-5.5

(assuming $30 \% \mathrm{H}_{2}$ concentration in the slurry gases)

\subsection{HEAT TRANSFER PROPERTIES AND ANALYSES}

The cyclical phenomena of slurry growth observed in tank 101-SY gas generation, temperature profile change, and subsequent reversion upon slurry settling and gas release have engendered much speculation (Jansky 1985). Various theories have been proposed, and causative mechanisms suggested, to explain these observations. This section addresses several relevant issues:

1) natural convection heat transfer taking place in the slurry, 2) the effect of gas bubbles on the slurry thermal conductivity, and 3) the effect of salts on thermal conductivity. 


\subsubsection{Natural Convection Heat Transfer}

Of interest here is whether natural convection can take place in the slurry that occupies the lowest $200 \mathrm{in}$. of the tank. In natural convection heat transfer, the Rayleigh number ( $R a)$, which serves as a measure of buoyancy force/viscous force, is an important parameter. Below a critical Ra, no convective mass transfer occurs, and therefore heat transfer is by pure conduction. At high $\mathrm{Ra}$, a second demarcation point is reached--the transition between laminar and turbulent flow.

Although the Ra is typically defined in terms of a temperature difference, it can also be defined for the slurry situation with volumetric heat generation (Goldstein and Kudo 1982):

$$
R a=g \beta / v \alpha(L / 2)^{3}\left(S L^{2} / 8 \alpha\right)
$$

$$
\text { where } \begin{aligned}
S & =\text { the volumetric heat production rate } \\
\beta & =\text { thermal expansion coefficient } \\
g & =\text { quantitative acceleration } \\
\alpha & =\text { thermal diffusivity } \\
L & =\text { length scale of apparatus. }
\end{aligned}
$$

Two Rayleigh numbers were calculated and are presented in Table 2.8. The first one $(\mathrm{Ra}=1040)$ used pure water properties evaluated at a typical slurry temperature of $60^{\circ} \mathrm{C}\left(140^{\circ} \mathrm{F}\right)$ (Weast 1984). The second $\mathrm{Ra}(\mathrm{Ra}=16.1)$ utilized measured slurry density (specific gravity $=1.69$ ) and viscosity (highest was $86.3 \mathrm{cP}$ ) according to L. M. Sasaki in a memo. The slurry generated about $22,300 \mathrm{Btu} / \mathrm{h}$ or a volumetric heating rate of $235 \mathrm{ft}-1 \mathrm{bf} / \mathrm{h}-\mathrm{ft}^{3}$ for a 200-in.-high slurry in a 75-fi-dia tank. The critical Ra was about 250 (Goldstein and Kudo 1982), so it is clear from Table 2.8 that natural convection is likely in water, but highly unlikely to be present in the slurry unless other forces (e.g., those due to bubbles) aid it. It can thus be concluded that the parabolic temperature profile observed prior to gas release is the result solely of conduction in the heat-generating medium. 
IABLE 2.8. Slurry Rayleigh Numbers (bounding)

\begin{tabular}{|c|c|c|c|c|c|}
\hline Fluid & $\begin{array}{l}\text { Density, } \\
1 \mathrm{bm} / \mathrm{ft}^{y}\end{array}$ & $\begin{array}{l}\text { Alpha, } \\
\mathrm{ft}^{2} / \mathrm{h}\end{array}$ & Viscosity, cP & Rayleigh No. & Comment \\
\hline Water & 61.4 & 6.15 & 0.4665 & $1.04 E+03$ & $\begin{array}{l}\text { Natural } \\
\text { convection }\end{array}$ \\
\hline Slurry & 103.77 & 3.64 & 86.30 & $1.61 E+01$ & No motion \\
\hline
\end{tabular}

\subsubsection{Thermal Conductivity of Slurry/Bubble Mixture}

The conductivity of the slurry must be known accurately because conduction is the dominant heat transfer mechanism. This conductivity needs to be formulated as a function of time, i.e., as a function of bubble generation rate, or total evolved gas volume present. The approach taken in calculating the conductivity was to first examine the $1 i$ terature for similar conditions.

A number of comparable mixed media diffusive phenomena have been analyzed and tested. For the special case (applicable here) of diffusion through a porous solid with periodically spaced spheres, the classic, exact solution of Maxwell is valid (Cussler 1984; Shulman 1983). The Maxwell equation is

$D_{\text {eff }} / D=\left[2 / D_{s}+1 / D-2 \phi_{s}\left(1 / D_{s}-1 / D\right)\right] /\left[2 / D_{s}+1 / D+\phi_{s}\left(1 / D_{s}-1 / D\right)\right]$

where $D_{\text {eff }}=$ the effective diffusion coefficient through the composite material

$D=$ the diffusion coefficient in the interstitial pores (i.e., continuous phase)

$D_{s}=$ the diffusion coefficient through the spheres (i.e., discrete phase)

$\phi_{s}=$ the volume fraction of the spheres in the composite (porous) material.

If diffusion through the spheres is negligible $\left(\phi_{s}=0\right)$ this equation reduces to:

$$
D_{\text {eff }} / D=2\left(1-\phi_{s}\right) /\left(2+\phi_{s}\right)
$$


If the diffusion through the spheres is extremely rapid $\left(D_{s} \rightarrow\right.$ infinity $)$ the solution becomes:

$$
D_{\text {eff }} / D=\left(1+2 \phi_{s}\right) /\left(1-\phi_{s}\right)
$$

The effective thermal conductivity (i.e., diffusion of heat) is calculated with each of the three equations in turn, and the results are provided in Table 2.9. In the second column, $\mathrm{H}_{2} \mathrm{O} / \mathrm{Sl}$ urry, the slurry is considered to be all continuous, with thermal conductivity equal to pure water at $140^{\circ} \mathrm{F}$. In the third column, $\mathrm{H}_{2} \mathrm{O} / \mathrm{N}_{2}$, the bubbles are assumed to consist entirely of $\mathrm{N}_{2}$ gas, which occupies $10 \mathrm{in.} / 200 \mathrm{in}$. of tank slurry height, so $f_{s}=0.05$. The fourth column, $\mathrm{H}_{2} \mathrm{O} / \mathrm{H}_{2}$, presents results based on bubbles consisting entirely of $\mathrm{H}_{2}$; note that the latest (1983) tests with synthetic slurry mixtures yielded gas compositions of about $64 \% \mathrm{~N}_{2}, 13 \% \mathrm{~N}_{2} \mathrm{O}$, and $21 \% \mathrm{H}_{2}$ (from a Westinghouse Hanford Company presentation). These calculations thus represent bounding-type results and indicate that the effect of gas composition is rather weak. In another set of extreme bounding calculations, shown in the last two columns of Table 2.9, the sphere/bubble conductivity is first assumed to be about zero, and then taken to be extremely large.

Another set of bounding calculations can be made using the assumption of either series or parallel resistive electrical network analogs (Tsao 1961; Brennan and Kroliczek 1979). Because the true slurry conductivity lies between these extremes, they represent a useful check on the earlier calculations. Results are shown in Table 2.9.

TABLE 2.9. Tank 101-SY Heat Transfer Calculations Effective Conductivity (Btu/h. $\mathrm{ft}$. of) Based on Composition

\begin{tabular}{|c|c|c|c|c|c|}
\hline Equation & $\mathrm{H}_{2} \mathrm{O} / \mathrm{ST}$ urry & $\mathrm{H}_{2} \mathrm{O} / \mathrm{N}_{2}$ & $\mathrm{H}_{2} \mathrm{O} / \mathrm{H}_{2}$ & $\underline{\mathrm{H}}_{2} \mathrm{O} / \mathrm{Vacuum}$ & $\mathrm{H}_{2} \underline{O}$ Infinite \\
\hline Maxwell & 0.380 & 0.354 & 0.363 & 0.352 & 0.440 \\
\hline Series & - & 0.179 & 0.340 & - & 0.400 \\
\hline Parallel & - & 0.362 & 0.367 & 0.361 & - \\
\hline
\end{tabular}


Other methods for determining the bubble/slurry mixture conductivity are avallable, but appear to be less relevant. Ratcliffe (196i8) presents a nomograph based on an extensive set of tests with two-phase media. Maddox (1983) suggests a procedure based on weight fractions that appears to be most app 1/cable to aqueous mixtures. Schunck (1983) provides a general approach and discussion on porosity and effects on conductivity in solids. Cheng and Natan (1986) give a numerical technique for calculating the electrical conductivity of a foam material, but because the gas phase is not a conductor, this method is not applicable here. Also considered was a method for calculating the effective conductivity among packed spheres (Bauer 1983; Kunil and Smith 1960), but this incorporated a contact resistance that is not present in a slurry/bubble inixture. Yet another approach for gas diffusion through pores considers tortuosity and pore size distribution in solls (Nielson, Rogers, and Gee 1984), but again is not applicable because diffusion exists in only one mixture component. Even less relevant are data and methods avallable for packed beds (Gnielinski 1983) and fluidized beds (Zabrodsky 1983; Martin 1987) where convective phenomena dominate. High-velocity air bubbles in glass melts (Ungan and Viskanta 1985) and attendant effects of bubbles on radiative transfer (Viskanta 1985) include phenomena that are of negligible importance here.

\subsubsection{Thermal Conductivity of Slurry}

The actual thermal conductivity of the slurry in tank 101-sy has not been measured. Based on slurry analysis of the bottom sample $e^{(a)}$ and the data given by Tsederberg (1965), it appears that the increase in conductivity from the $\mathrm{NaOH}$ would be approximately cancelled by the decrease due to $\mathrm{NaNO}_{2}$ and $\mathrm{NaNO}_{3}$. Armstrong and Winter (1983) provide limited data and polynomial equations for temperature-dependent property variation of non-Newtonian fluids, but give little guidance on how a slurry such as in 101-sY might behave.

(a) L. M. Sasaki, Rockwe11 International, memo. 


\subsection{PHOTOGRAPH DESCRIPTION AND ANALYSIS}

A panorama sertes of photographs of the inside gas space above the waste crust of tank 101-SY was taken in April 1989. The photos are in color and show a relatively rough top surface of the crust. Evidence exists of liquid flow onto the crust surface and of gas-blown effluvia on the walls and other structures that hang in the tank. Different people have interpreted some features differently. For example, some interpret a brown band of color near the wall as the edge of a crust shelf, suggesting that the crust is bonded to the wa11. Others do not interpret this color change as a shelf, but a pattern of 1iquid ejecta. An analysis of the photographs was begun in order to quantify the locations of various features of the crust and to determine the direction and angle to which some probes have been bent. In the photos for example, the thermocouple probe is seen to be bent to an angle of about $10^{\circ}$ from vertical. The direction cannot be determined by simply viewing the photograph print because the angle may not be perpendicular to the camera axis. It is important to know the angle because with the probe not vertical, the as-built elevations of the thermocouples are not correct. The temperature profiles need to be adjusted in height to take this into account (see Section 2.10.1).

\subsubsection{Photographic Analys is Procedure}

Some qualitative observations from the photographs include the following:

- The crust level at the wall is slightly (1 to 2 in.) below the lower tangent weld of the upper knuckle. However, there appear to be some regions of the crust rim that are higher than that level.

- White spume marks are dried onto the wall at various places around the crust rim. Some particles have gone as high as $3 \mathrm{ft}$ above the crust. Similar marks and ejecta are seen on lances that penetrate the crust.

- Dark areas, suggesting a liquid flowing out over the top of the white crust, are seen near the bent lances.

- Cables and lances have been moved from the vertical locations below their respective risers.

The following steps are used in the quantitative analysis of the photos: 
1. Estabilisle camera shot azimuths. The pictures were taken through riser 13ee Figure 2.1), not at the center of the vesse1. A first panorama 12 shots was taken at a tilt angle of about $20^{\circ}$. By comparing tlamera scenes with the as-butlt drawings of the tank, matching same features, and correcting for the aberration of the lens useine polar angle of each shot was determined and is given in Table?.

2. Establisle camera height and tilt angle. The analysis of the photos continueising the upper knuckle weld line on the tank as a reference point toermine the height of the camera in the tank and the tilt angle of first sertes of 12 photos. See Table 2.10.

3. Establisle level of crust where it meets the wall for each of the photos. s calculation depends on the previously established data for each shod on the distance to the wall where the crust contacts it.

4. Establisle location of surface features on a plan view. Assuming that thest is level (or nearly level), this can be done. For example, the ation of the tilted probe entry point into the crust can be found ane tilt angle from the header entry for the probe can be calculated.ble 2.10 shows the calculated tilt azimuth and angle off vertical two lances and the thermocouple probe. The location determined fortain color patterns will help determine if they represent flutd flatterns or crust shelves.

IABLE 2.10. Waste Tank Photo Analysis

$\begin{array}{cccccc}\text { Photo } & \begin{array}{c}\text { ar } \\ \text { Angle, rees (a) }\end{array} & \begin{array}{c}\text { Camera } \\ \text { Iilt, degres }\end{array} & \begin{array}{c}\text { Riser } \\ \text { Number }\end{array} & \begin{array}{c}\text { Polar } \\ \text { Angle, degrees }\end{array} & \begin{array}{c}\text { Angle } \\ \text { Bent. degrees. }\end{array} \\ 2 & 4 & 21.5 & & & \\ 3 & 1 & 17.9 & & & \\ 4 & 34 & 18.2 & 17 C & 227.7 & 23.9 \\ 5 & 28 & 21.6 & 4 \mathrm{~A} & 19.4 & 8.1 \\ 6 & 25 & 21.2 & 1 \mathrm{~A} & 239.5 & 26.0 \\ 7 & 22 & 19.9 & & & \\ 8 & 19 & 21.2 & & & \\ 9 & 15 & 20.2 & & & \\ 10 & 12 & 19.9 & & & \\ 11 & 9 & 21.2 & & & \\ 12 & 7 & 20.5 & & & \end{array}$

(a) Polar anis measured in counterclockwise direction from a zero angle at the gigree tank azimuth. 
5. Using mutual features on the surface, establish the azimuth and tilt angle of the second ring of panorama photographs, and from that the third set can also be similarly callbrated.

6. Establish the crust surface features of interest on a plan view drawing from any of the calibrated photographs.

\subsubsection{Thermocouple Probe Elevations}

The photographic analysis has shown that the probe is bent at an angle of at least $8^{\circ}$. The angle may be less if the plastic bending has formed a radius of bend below the riser entry point rather than the sharper angle assumed.

Table 2.2 gives the as-butlt elevations of the probe thermocouples 101-1 through 101-18 versus the more likely height off the bottom due to the tilt of the probe. All graphic plots of the temperature profiles that have been received from Westinghouse Hanford Company should be corrected as suggested in the table to give a better representation of the physical location of the temperature profile.

Figure 2.19 is an example of the prerelease proftle of October 23, 1990, as given and as corrected for the thermocouple tilt. 


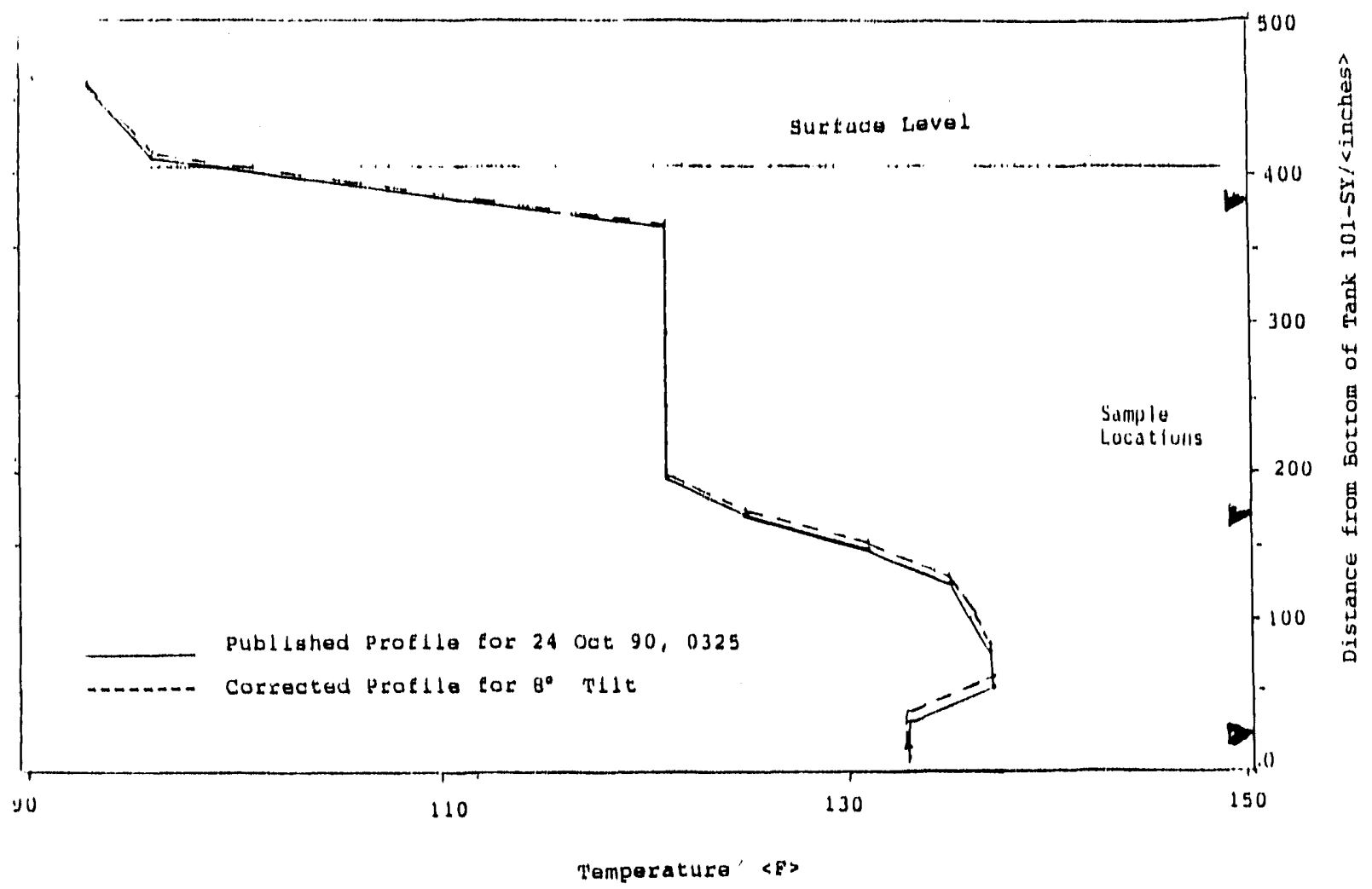

FIGURE 2.19. Effect of Tilt of Thermocouple Probe on Temperature Profile 


\subsection{RECOMMENDATIONS ON DATA GATHERING}

Temperatures of tank 101-SY and other tank contents indicate waste status and are being used to infer $\mathrm{H}_{2}$ production and slurry growth/collapse. It appears fatrly clear that a coupling exists between observed temperature profiles, $\mathrm{H}_{2}$ production and release, and slurry growth as indicated by crust motion. What is not clear is the exact nature of this coupling, and any potential cause-and-effect mechantsms cannot be deduced based on current data sampling methods (f.e., weekly temperature recording).

Data we have obtained thus far from tank 101-SY instrument readings are inadequate in several respects:

- Only some of the instruments/sensors are read (this is espectally true for thermocouples).

- Readings are manual, and rounded off in some fashion.

- Reading frequency is low.

In order to allow us to model the observed behavior in tank 101-SY, it is suggested that the following actions be taken:

- All functioning instruments should be read/recorded.

- Data stored by the data logger/data acquisition system should be directly accessible.

- Dally readings, taken over the period of about a week, should be taken. Readings every 10 seconds, as on October 24, 1990, should be continued in future releases.

Such actions will both improve our understanding of tank 101-SY phenomena and permit assessment of additional instrumentation/sampling needs. Because of the importance of thermocouple readings in slurry behavior models, it is strongly recommended that readings be automated via a data logger, and the frequency be increased to hourly $\left(\mathrm{m} / \mathrm{n}^{-1}\right.$, or $15 \mathrm{~m}^{-1} \mathrm{n}^{-1}$ preferred). A11 these data need not be saved; a data buffer plus appropriate "trigger" setpoints could preserve data around critical events only. 


\subsection{SAMPLE DATA}

Samples of waste taken from the prerelease layers need to be measured for composition, viscosity, specific gravity, yield strength, and shear strength. Also needed are in situ measurements of bubble sizes and slurry properties.

\subsection{PHOTOGRAPHY}

Additional photographs to show changes and siereo photographs should be taken in the tank; videos should be made in the tank during a release. 


\subsection{DEFINITIONS OF MECHANISTIC MODELS FOR TANK 101-SY GAS RELEASE}

In discussions of the tank 101.SY gas release phenomena, several physical mechanisms have been proposed that might describe what is actually happening in the tank to produce the sequences of temperature profiles, level swells, and gas releases that are being measured. The basic phenomenon that the mechanistic model is to explain is the periodic release of gas. The mechanism of production of the gas is thought to be chemical and/or radiological and is assumed to be steady and continuous. This seems a reasonable assumption because the level in the tank is observed to rise until a relatively sudden release of gas and an associated drop in level. It is not likely that the reaction would suddenly produce the large quantity of gas that is released in a few hours. This section of the report categorizes the mechanisms or models in order to clarify discussion, analysis, and future measurements concerning them. The mechanisms have been given an alpha-numeric nomenclature to speed communication.

\subsection{MODELS FOR GAS HELD BY THE CRUST}

These models are based on the idea that the gas is trapped by a relatively impervious crust at the top of the waste in the tank. Periodically the crust breaks in some way to release the gas.

\subsubsection{Model C-1 (Crust Fracture)}

The crust is in the form of a flat plate that is supported by a flat bubble of gas directly underneath the crust but over the liquid. The gas gathers in the flat bubble, raising the crust. At the time of release, the crust fractures, allowing the gas to escape, the crust to descend (at least at the place where the level is measured), and to reform a gas seal.

\subsubsection{Model C-2 (Crust Tilt)}

The crust is a flat plate riding on the gas bubble atop the liquid in the waste tank. At gas release, the crust tilts, allowing the gas to escape in a short time. The crust then levels and reforms a gas seal. 


\subsection{MODELS FOR GAS HELD IN SLUDGE}

This concept was proposed by Reynolds(a) as an alternative to the underthe-crust idea. Therefore these models are called Reynolds models. In these models the crust does not offer significant resistance to the release of the gas.

\subsubsection{Model R-1 (Total Sludge Buoyancy)}

Reynolds ${ }^{(a)}$ describes this model:

When the gas is generated it is being generated in the bottom of the tank. It is held in the lower 200 in. in the settled solids zone. As the bottom of the tank heats up, the gas generation rate increases, which produces more gas. When finally the gas held in the settled solids zone gets large [enough,] <that> the buoyancy forces overcome the inertia and viscosity of the bottom zone. This lifts the material up to the top of the tank where the gas can escape. The lifting of the material also relieves the pressure on the gas so the gas expands, which increases the buoyancy, which hastens the rollover in the tank. In the end, the tank contents have been mixed and are uniform temperature and the gas has been released. The solids that were brought to the top of the tank start to settle. When the solids pile on the bottom, they inhibit convection currents and the solids pile begins to heat up. Heating accelerates the gas generation and once again the process begins.

The essence of this model is that the gas formed in the sludge makes the entire sludge rise, and the separation of the bubbles from the solids occurs high in the tank pool.

\subsubsection{Model R-2 (Gas Supersaturation)}

This model is also described specifically by Reynolds. "The other potential mechanism is that this is similar to the "tank bump" but on a less energetic scale. Instead of enough heat being trapped to generate steam, the gas forms the mechanism for release." The essence of this model is that the gas is collected at the bottom in a supersaturated condition. Some trigger causes a local or general reduction of pressure, which allows the gas to nucleate into bubbles that grow rapidly and rise from the sludge and fluid mass.

(a) D. A. Reynolds. Westinghouse Hanford, internal memo. 


\subsubsection{Model R-3 (Bubble Separation from Sludge)}

In this model the gas that is periodically released is formed and collected as small bubbles in the thick solid-liquid sludge layer near the bottom of the tank. The bubbles are held in the sludge by surface adhesion to the solids or by the sludge viscosity and yield strength. The bubbles grow in size until they can no longer be held by these forces. Some bubbles begin to rise and the motion aids and triggers the release of other bubbles. The triggering could be local and could progress as a wave across the tank, or it could be at some general level in the waste that progresses to other levels above or below. The accelerated mass motion then raises some of the solid as we11, allows liquid to descend, and equalizes the temperature. The essence of this model is that the bubbles in the bottom start separating from the waste sludge at the bottom of the tank.

\subsubsection{Model R-4 (Local Gas Bubble)}

In this model the tank is not assumed to be uniform across the bottom. Some of the gas that is formed gathers in a large bubble under a membrane-1ike layer of sludge. Eventually the membrane breaks, and a large bubble of gas rises from this local part of the tank, passes through the liquid quickly, and impacts against the crust. This model may suggest that the location of the bubble formation and release is where the thermocouples are. The liquid fills in the cavity where the bubble was and flattens the temperature profile at the thermocouple stalk. The essence of the model suggests that a large bubble is located where it will affect the thermocouple readings.

\subsubsection{Model R-5 (Local Buoyancy Upset)}

Mode1 R-5 was suggested after preliminary review of the temperature data from the Tank 101-SY gas release of October 24, 1990. These data show a warm temperature just below the crust immediately after the "burp." This temperature bulge in the vertical temperature profile from the thermocouple tree is roughly equal to the temperature bulge low in the tank before the burp. This behavior suggests that a large amount of the bottom sludge material has risen 
to the top accompanying the gas release, and cooler fluid has come in below this sludge (with some mixing perhaps) from its previous location above the sludge.

The hypothesis of this model is that a part of the sludge has collected enough gas to become less dense than the fluid above and achieves enough buoyancy to rise, releasing trapped gases. The risen sludge, by holding some gas or by sticking to the thermocouple probe, falls slowly back to the bottom layer where long-term heating begins again. The major feature of this model is that the sludge is made buoyant in only a part of the entire tank, which happened to be in the region of the thermocouple tree at least for the October 24, 1990, event. In an earlier case, a high temperature was noticed just under the crust, but only one thermocouple gave this reading and it was not verified by other data.

\subsubsection{Model R-6 (Hot Liquid Release)}

In this model, the formation and release of gas opens pathways for hot liquid to rise from the sludge. The liquid, by being heated, is less dense than the liquid above but has been held down by the cohesive slurry. Cooler liquid from above fills in regions where the hot liquid was. As the liquid rises it brings some sludge solids with it. These later settle to the bottom again and begin to recreate the bottom bulge of temperature. In this theory the probe thermocouple behavior might also be explained by vibration of the thermocouple stalk. The stalk is vibrated by the fluid motion created by the rise of the bubbles and liquid. The movement allows cooler liquid to come down along the sides of the stalk, but the small amount of liquid reheats rapidly because the hot, nonconvected sludge located close by reheats it and the thermocouples. 


\subsection{CRUST STRENGTH CALCULATIONS}

The primary motivation for the study of crust structural response due to pressurization from beneath is to assess the likely location for gas escape as pressure increases. Stress levels were estimated for the body of the crust as we11 as the crust-tank interface.

\subsection{CONCLUSIONS}

Stress levels in the body of the crust (away from the tank interface) were found to be dominated by simply supported plate behavior. The maximum stress level was found to exist at the center of the crust and be approximately 108 times $P_{\text {eq }}$, the equivalent pressure beneath the crust. Stresses at the crust-tank interface were much smalier than those in the body of the crust. Vertical shear stress was found to be 4.68 times $P_{\text {eq }}$. Edge flexure stress caused by the rotational stiffness of the liner shell was found to be approximately 4.5 times $P_{\text {eq }}$.

Use of these calculations will require knowledge of the strength properties of the crust and the crust-tank bond.

\subsection{DISCUSSION}

Stress levels were normalized with respect to the net equivalent pressure load. This net equivalent pressure load $\left(P_{e q}\right)$ is the actual pressure in the gas bubble beneath the crust minus the pressure required to balance the weight of the crust (about $2.6 \mathrm{psi}$ ) and minus the gas pressure above the crust. Material properties and model geometry used in this investigation were taken from information compiled by Westinghouse Hanford Company relating to the hydrogen burn issue. These data are summarized in Table 5.1.

Calculations were first performed to assess the relative rotational stiffnesses of the cylindrical shell representing the tank and the 48-in.thick, 900-in.-dia plate of crust material. Equations used for these calculations for the crust and for the tank wall can be found elsewhere (Timoshenko and Woinowsky-Krieger 1959; Roark and Young 1975, respectively). The crust was found to be much stiffer than the thin tank wall. For this 
TABLE 5.1. Material Properties and Geometry Nomenclature

$$
\begin{aligned}
& E_{c}=\text { crust Young's modulus--2.9E4 psi (calculated } \\
& \text { from density and sonic velocity data) } \\
& E_{s}=\text { shell Young's modulus--30 E6 psi (standard data) } \\
& t=\text { crust thickness }(48 \mathrm{in} .) \\
& R=\text { crust radius (450 in.) } \\
& r=\text { distance from crust center (in.) } \\
& P_{\text {eq }}=\text { pressure beneath crust minus equivalent pressure } \\
& \text { of crust weight and minus pressure above crust } \\
& \text { (psi). }
\end{aligned}
$$

reason, stress levels in crust can be calculated by assuming the crust behaves as a simply supported circular plate. The expressions for hoop and radial flexure stresses for this case are taken from Timoshenko and Woinowsky-Krieger (1959):

$$
\begin{aligned}
& \frac{\sigma_{\theta}}{P_{\text {eq }}}=\frac{3}{8 t^{2}}\left[R^{2}(3+v)-r^{2}(1+3 v)\right] \\
& \frac{\sigma_{r}}{P_{\text {eq }}}=\frac{3}{8 t^{2}}(3+v)\left(R-R^{2}\right)
\end{aligned}
$$

$$
\text { where } \begin{aligned}
R & =\text { crust radius }(450 \text { in. }) \\
t & =\text { crust thickness ( } 48 \text { in.) } \\
r & =\text { distance from crust center } \\
& U=\text { crust Poisson's ratio }(0.3 \text { assumed }) .
\end{aligned}
$$

These stresses are illustrated in Figure 5.1. It is interesting to note that both hoop and radial stresses are tensile on the top surface and compressive on the bottom with peak values occurring at the center. In addition, 


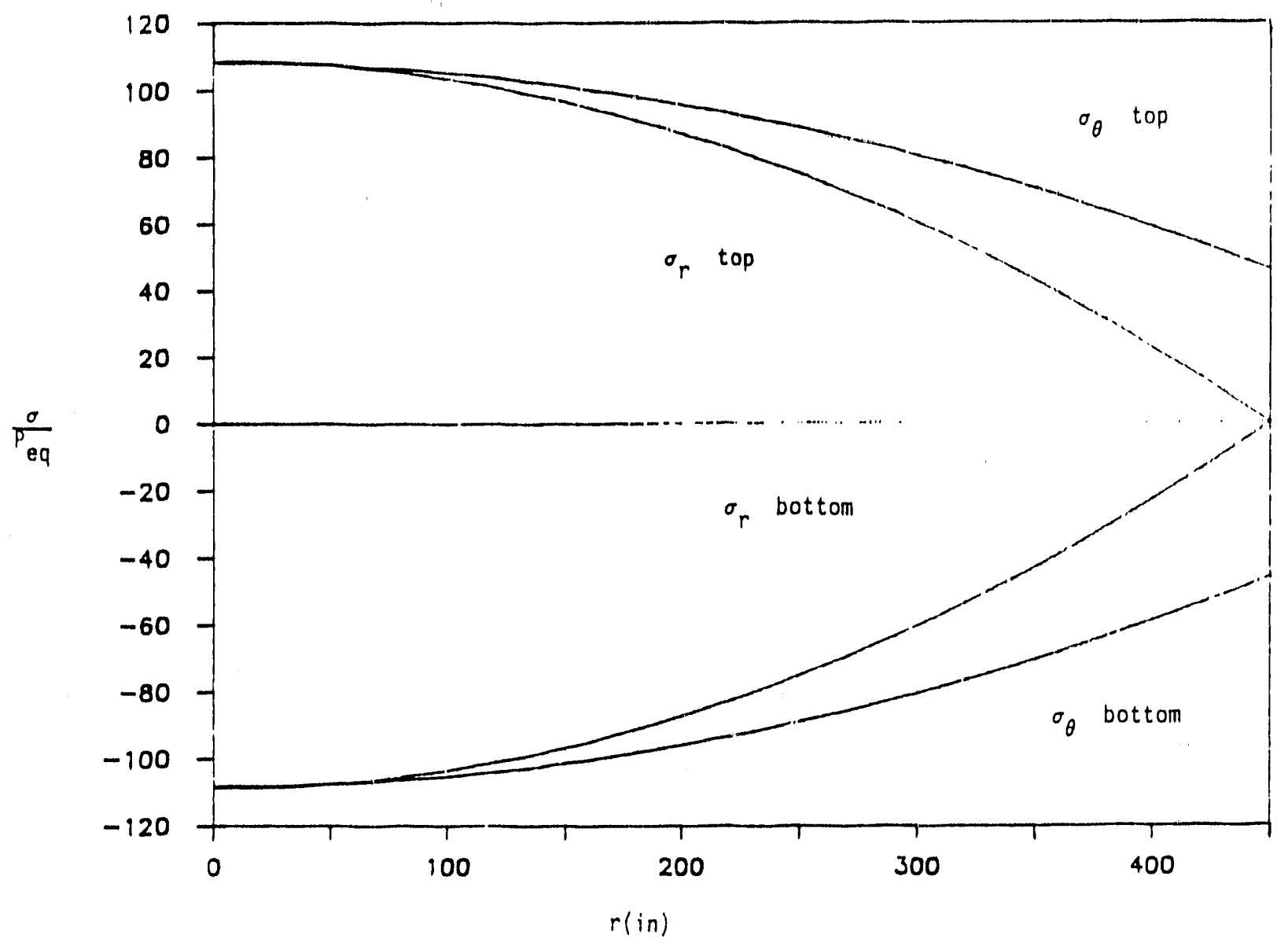

FIGURE 5.1. Crust Stresses Due to Bottom Pressure

these stress levels are not dependent on Young's modulus of the crust (a rather uncertain material property).

Flexure stresses computed in this fashion are essentially in agreement with those computed by a Westinghouse Hanford Company employee and described in a letter. (a)

Stress levels at the crust-tank interface consist of the following two components:

- a vertical shear stress equilibrating the pressure force

(a) Letter from C. L. Moore to N. W. Firch, both Westinghouse Hanford Company, July 26, 1990. 
- a flexure component due to the rotation of the crust edge and the rotational stiffness of the tank.

These components were found to be much smaller than stress levels in the center of the crust.

The average shear stress through the thickness of the interface is computed from equilibrium considerations:

$$
\frac{\tau}{P_{\text {eq }}}=\frac{R}{2 t}=4.68
$$

The flexure component of stress at the crust-tank interface is caused by the rotational stiffness of the tank wall. Using formulas from Timoshenko and Woinowsky-Krieger (1959) and Roark and Young (1975), a conservative estimate for this flexure stress (expressed nondimensionally) is

$$
\frac{\sigma}{P_{\text {eq }}}=4.5
$$

Since this is a flexure stress, a compressive component of the magnitude given by the above expression will exist on the top of the crust-tank interface, and a tensile component will exist on the bottom. This value, strictly speaking, should be added to the flexure stresses shown in Figure 5.1 , but the effect would be negligible in view of the modeling assumptions. 


\subsection{OVERTURN CALCULATIONS}

The purpose of this section is to crittcally examine a physical theory set forth to explain the gas release events in tank 101-SY. It has been suggested that the gas is released from tank 101-SY when the lower layers of the tank contents turn over due to density instability. The theory suggests that bubbles are trapped in the lower regions of the tank and lead to the expansion of the lower layer of slurry. In time, the lower layer expands sufficiently to cause the slurry density to fall below that of the overlying supernatant fluid. Gas release occurs when the two layers overturn as a result of this density inversion. This theory is referred to as Reynolds' R-1 model in Section 4.2.1.

In order to be plausible, this theory and some others require some means of gas entrapment in the slurry. The stressustrain curves obtained from samples of tank 101-SY slurry suggest that the material in the bottom of the tank exhibits a yield stress. (a) Materials that exhibit a yield stress are capable of immobilizing small particles or bubbles and would be expected to trap gas. Thus it does appear posstble that gas could be trapped in the lower slurry layer.

\subsection{SUMMARY}

An analysis based on the measured expanstion and the specific gravity of the tank contents was performed to determine if the R-1 model was plausible. An analysis based on measurements of the specific gravity of the tank contents suggests that the R-1 model is not consistent with the magnitude of level changes that occur during a gas release. In this analysis the change in density of the lower slurry layer as a result of tank expansion was determined, and the result was compared to the change in density required to allow the level drops that occur to be explained under this theory.

The result of this analysis on the basis of the reported specific gravities contradicts that of Epstein and Fauske (letter), who indicate that the

(a) B. M. Mauss. Rockwell International, internal letter. 
R-1 theory is posstble. We belleve that the Epstein and Fauske analysis is not valid for the following reasons:

- Epstein and Fauske assumed that the speciftc gravity of the lower layer of slurry was 1.49 . The specific gravity measured for the lower layer was 1.69 per Mauss.

- Using the low estimate for the specific gravity of the slurry, Epstein and Fauske concluded that the level of slurry expansion requitred to destabilize the lower layer was 22 in.; this was interpreted to indicate that the mechanism was realistic. Level drops have occurred when the slurry is expanded only $5 \mathrm{ln}$., which is a significantly smaller level of expansion. Thus, the mechanism does not appear to explain this level drop or a number of other observed level drops.

- Epstein and Fauske did not explicitly define their variables. Their analysis appears to use inconsistent definitions of the gas volume fraction and the porosity.

\subsection{GENERAL ANALYSIS}

Schematic diagrams of an expanded and unexpanded configuration for the tank are shown in Figures 6.1 and 6.2 , respectively. In the expanded configuration, the lower layer is assumed to be composed of slurry that has been expanded by trapped bubbles and has a spectfic gravity of $p_{s 1}$. The upper layer is composed of an unexpanded material with a liquid specific gravity of $\rho_{1}$. The total height of the tank contents is $H_{1}$, and the height of the slurry is $h_{s 1}$.

It is assumed in this analysis that the lower layer expands uniformly from top to bottom. It has been suggested by Epstein and Fauske in a letter that the hydrostatic pressure differences at the top and bottom of the siurry layer cause the upper layer to expand a greater amount than the lower layer. The magnitude of this effect will be addressed at the end of this analysis.

In the unexpanded configuration, the height of the total tank content is $\mathrm{H}_{2}$, and the height of the lower slurry layer is $h_{s 2}=x_{2}$, where $x$ is the fraction of the tank occupled by the slurry layer. The specific gravity of the upper portion of the tank contents is $\rho_{1}$, as in the expanded configuration; the specific gravity of the unexpanded lower layer is $\rho_{\mathrm{s} 2}$. 


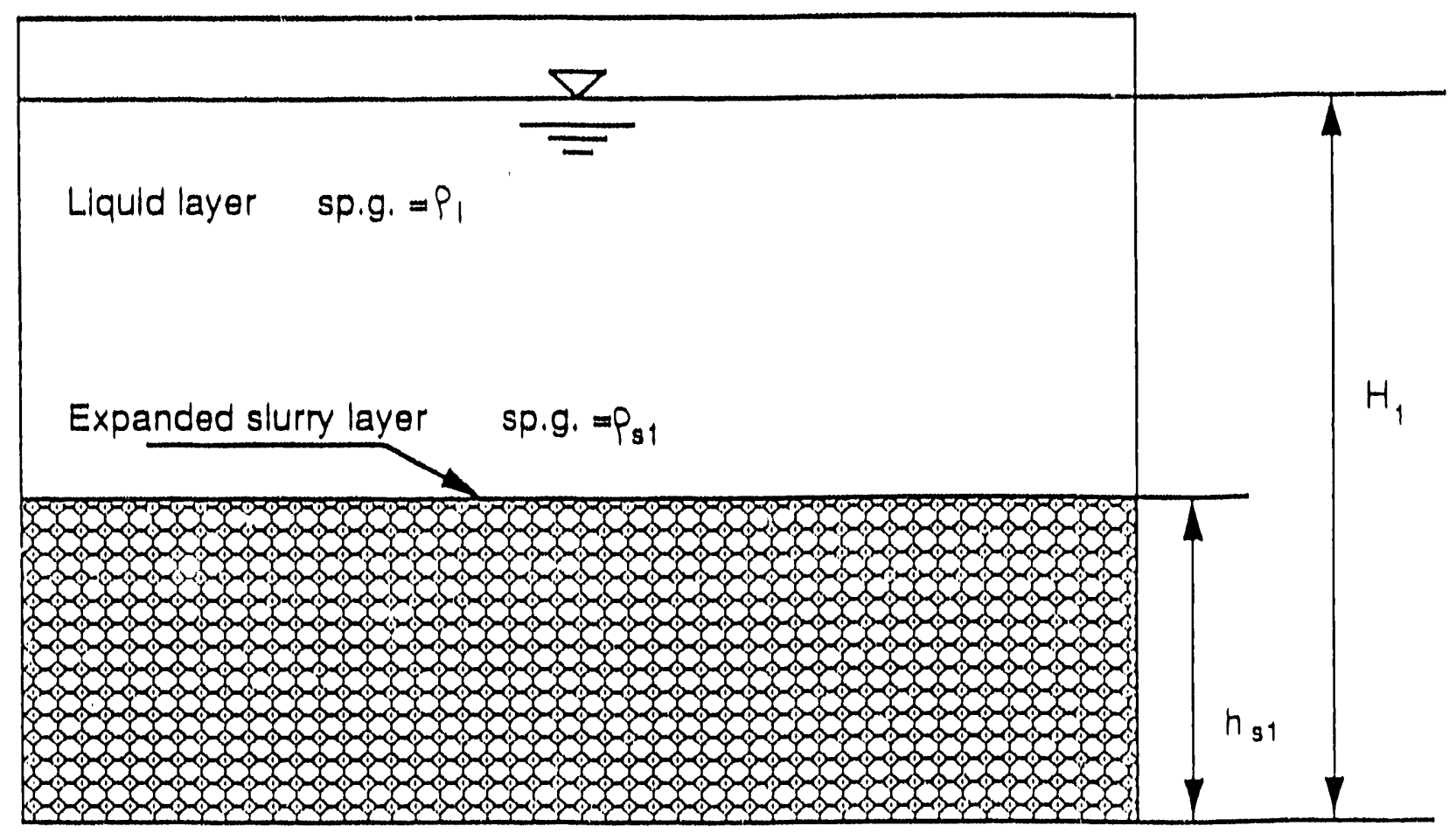

EIGURE 6.1. Expanded Tank Configuration

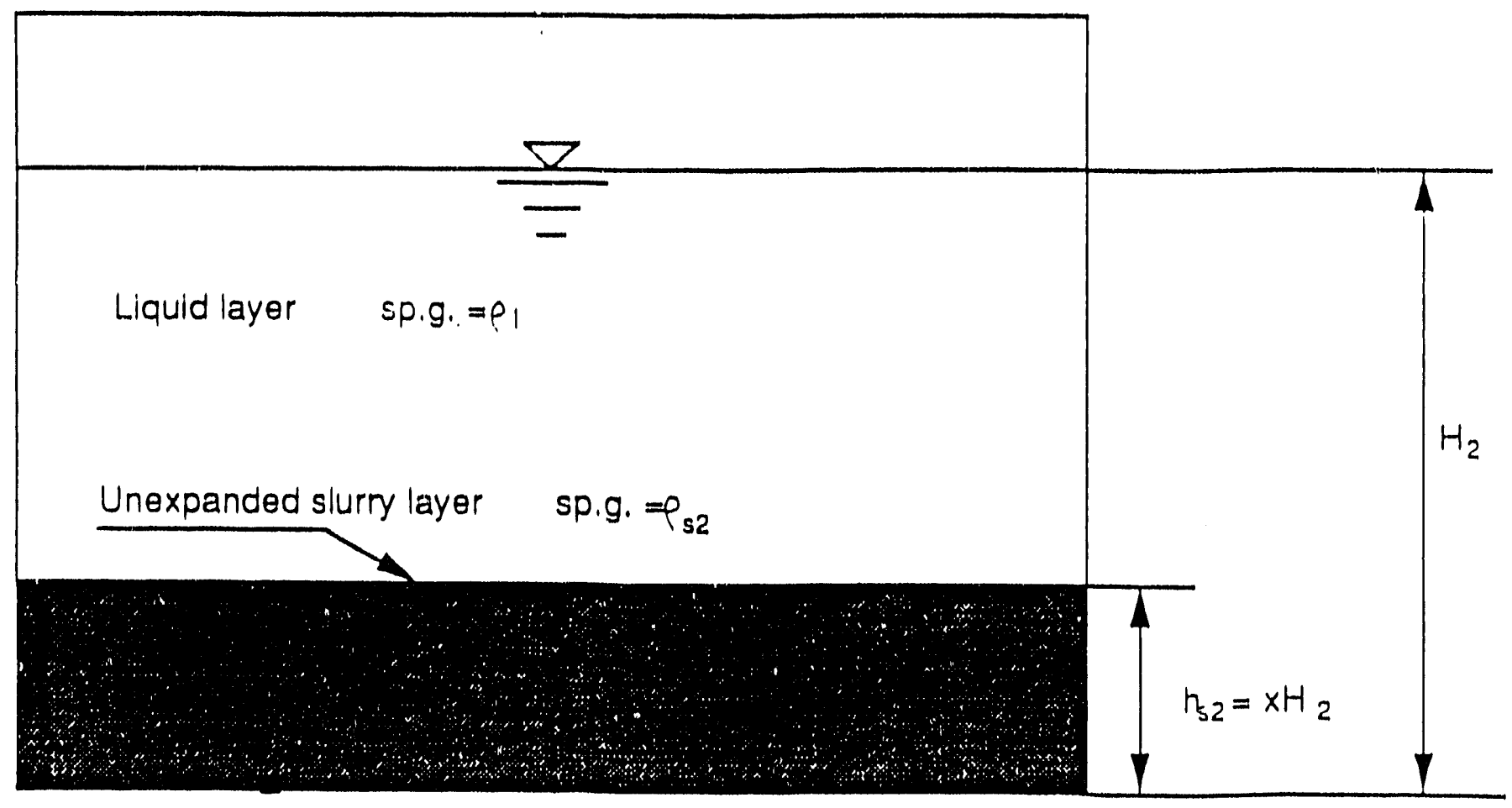

FIGURE 6.2. Unexpanded Tank Configuration 
The change th height of the tank contents that occurs during a level drop may be determined from the helght before and after the level drop:

$$
\Delta h=H_{1}-H_{2}
$$

Since all of the expansion is expected to occur in the lower slurry layer only, the height of the expinded slurry is

$$
h_{s 1}=h_{s 2}+\Delta h=x H_{2}+\Delta h
$$

Gas is generated in the slurry layer as a result of some chemical reaction and radiolysis. The entire quantity of gas trapped by the slurry is assumed to be expelled during a level drop, and the mass of gas trapped is assumed to be negligible with respect to the mass of the slurry in the lower layer. Thus the mass of expanded slurry will be identical to the mass of the unexpanded slurry. The density of tha expanded slurry is

$$
\rho_{s 1}=M_{\text {slurry }} / V_{\text {slurry, } 1}
$$

where $M_{\text {slurry }}=$ the mass of the slurry

$V_{\text {slurry, }}$ = the volume occupled by the expanded slurry.

The density of the unexpanded slurry is

$$
\rho_{s 2}=M_{\text {slurry }} / V_{\text {slurry,2 }}
$$

where $V_{\text {slurry,2 }}=$ the volume of the unexpanded slurry.

Since the volume is proportional to the height, the ratio of the expanded slurry density to the unexpanded slurry density is

$$
\frac{\rho_{s 1}}{\rho_{s 2}}=\frac{h_{s 2}}{h_{s 1}}=\frac{h_{s 2}}{h_{s 2}+\Delta h}=\left(1+\frac{\Delta h}{h_{s 2}}\right)^{-1}
$$


Substituting in terms of the total unexpanded tank height results in

$$
\frac{\rho_{s 1}}{\rho_{s 2}}=\left(1+\frac{\Delta h}{h_{s 2}}\right)^{-1}
$$

The ratio of the spectfic gravity of the slurry after expanston to the spectfic gravity of the overlying flutd will be called the stabtlity ratto, $\sigma$, and can be shown to be equal to:

$$
\sigma=\frac{\rho_{s 1}}{\rho_{1}}=\frac{\rho_{s 2}}{\rho_{1}}\left(1+\frac{\Delta h}{X H_{2}}\right)
$$

When the stability ratio falls below one, the density of the fluid overlying the slurry, $\rho_{1}$, is greater than that of the slurry, $\rho_{s 1}$, and the slurry may overturn. Large values of the stability ratio indicate a very stable configuration.

It is clear on the basis of Equation (6.7) that the greatest destabilization occurs when the level change in the tank is largest. However, in order for the theory being discussed to be plausible, it must explain all gas release events of this type that have been recorded. Thus, evaluation of the theory should be made on the basis of the smallest level change at. which a level drop has been observed to occur.

\subsubsection{Assumptions Based on Published Tank Data}

The data for the unexpanded tank condition are taken from tank height reading data presented in Panjunen et a1. (1990), which are shown in Figure 2.5. The minimum height recorded is assumed to correspond to the unexpanded tank contents height. The minimum tank level recorded since 1981 is approximately 403 in., which occurred late in 1984 . It is assumed that this corresponds to completely unexpanded tank contents. Estimates of unexpanded tank level based on the volume of additions to the tank suggest that the unexpanded tank contents are only $387 \mathrm{in}$. according to a letter by D. P. Simpson, which would suggest that the tank conterits are always slightly expanded. 
However, there is no indication to suggest that this minimum estimated tank level was ever achieved. In additioli, the estimate of the tank level appears to assume that the tank contains no internal components that would occupy a significant portion of the tank volume. Thus the level of $\mathrm{H}_{2}=403 \mathrm{in}$. appears to be the best estimate of the unexpanded tank contents. Because the estimate of the unexpanded tank level has a strong influence on the results, the stability estimate will be performed for both values of the minimum height.

The iiinimum tank level at which tank level drops have occurred appears to be $\mathrm{H}_{2}=408 \mathrm{in.}$, which occurred early in 1982. The minimum expansion of the tank contents at which a level drop has occurred appears to be $\Delta h=5$ in. if the minimum tank level is assumed to be $403 \mathrm{in.}$ and $21 \mathrm{in}$. if the minimum tank level is assumed to be 387 in.

The fraction of the tank occuijied by the unexpanded slurry layer is not well known. Temperature data suggest that some interface may exist near thermocouple 101-8, which is approximately $172 \mathrm{in}$. from the bottom of the tank, Figure 2.11. This would suggest that the bottom slurry occupies approximately $40 \%$ of the tank. However, it is assumed for the purpose of this calculation that the bottom slurry occupies only one-third of the tank contents. Examination of Equation (6.7) indicates that a smaller slurry height enhances the destabilizing effect of the expansion. Thus, if the theory is shown to be implausible using $x=0.33$, it will also be implausible using $x=0.4$.

Prior to expansion by gas entrapment, the specific gravity in the lower region of the tank is assumed to be 1.69 . The specific gravity of the overlying liquid layer is taken as 1.33. These values correspond to the specific gravities reported for a "bottom" and a "top" sample taken from tank 101-SY (Mauss internal letter). It should be noted that the "top" sample analyzed by Mauss was a slurry that contained $45 \%$ settled solids and $18.7 \%$ filtered solids. The supernatant in this sample had a specific gravity of 1.30 . It is prssible that the specific gravity of the fluid layer in the tank was somewhat" smaller than the specific gravity of the "top" sample analyzed by Mauss. The middle sample was reported to have a specific gravity of 1.29 , and the specific gravity of the supernate of the middle sample was 1.17 . Use of a lower 
value of the specific gravity for the liquid leads to larger values of the predicted stability ratio, which indicates, greater stability.

The magnitude of all independent and dependent quantities discussed here are shown in Table 6.1. Since density is proportional to specific gravities, the ratio of the densities after expansio' is equal to the ratio of the specific gravities [found using Equation (6.7)]. If it is assumed that the minimum tank level is 403 in., the stability ratio is $\sigma=\rho_{s 1} / \rho_{1}=1.22$; if the minimum tank level is $387 \mathrm{in.}$, the stability ratio is $\sigma=1.09$. In either case, the stability ratio is significantly greater than one and suggests that the slurry would not overturn at the level of expansion assumed here; yet level drops occur at this magnitude of expansion. Thus the model, as proposed, appears inconsistent with the available data.

\subsubsection{Modifications to Assumptions of Overturn Theory}

It should be noted that the theory may be plausible if some modifications are made. For example, if the slurry occupies a much smaller fraction of the tank contents, or if the gas entrapment is localized, the reduction in the slurry density in the region in which gas is trapped may be much greater than predicted here, and could result in instability. However, some justification for more localized gas entrapment or for a shallower slurry layer would be required, particularly since the flat temperature profile observed after the tank level drops suggests that the entire slurry layer is involved in the

TABLE 6.1. Tank Heights and Specific Gravities Used to Test the Tank Destabilization Theory

Unexpanded height of tank contents $\left(\mathrm{H}_{2}\right)$

Expanded height of tank contents $\left(H_{1}\right)$

Change in height due to expansion ( $\Delta h$ )

Fraction of tank occupied by unexpanded slurry $(x)$

Specific gravity of the unexpanded slurry $\left(\mathrm{sp} . g \cdot{ }_{2}\right)$

Specific gravity of the upper layer (sp.g., )

Ratio of the density of the unexpanded slurry

to the density of the liquid layer $\left(p_{s 1}, p_{1}\right)$

$\begin{array}{rrr}\text { Case 1 } & & \text { Case 2 } \\ 403 \text { in. } & 387 \text { in. } \\ 408 \text { in. } & 408 \text { in. } \\ 5 \text { in. } & 21 \text { in. } \\ 0.33 & 0.33 \\ 1.69 & 1.69 \\ 1.33 & 1.33 \\ 1.22 & 1.09\end{array}$


gas entrapment and release mechanism. If the volume of slurry in which gas is trapped is greater or equal to that used in this analysis, the type of destabilization mechanism described here is not possible.

The theory might also be deemed plausible if the average specific gravity of the lower layer of slurry is significantly lower than the specific gravity used in this analysis, or if the specific gravity of the upper layer of fluid is larger. The values used were those reported by Mauss; however, the tank levels from which these samples were drawn were not reported. Consequently, it is possible that these values cannot be applied to determine the density of the lower slurry layer or the density of the upper supernatant layer. These possibilities can be resolved by drawing new samples and measuring their specific gravity.

A final challenge to this theory, and all others proposed, is the need to explain the fact that the tank contents do not fall back to their original level after each gas release. Figure 2.5 indicates that in late 1988 and early 1990 the tank level after gas releases was $414 \mathrm{in.}$, which is much greater than those levels after previous level drops had occurred. The destabilization theory discussed here and the temperature profile data appear to suggest that the upheaval leads to motion and mixing of the lower layers of the tank. However, the fact that the tank level does not return to the same height after each level drop suggests that some gas remains trapped after each level drop. The proposed theory does not describe how some gas could remain trapped somewhere in the tank after a level drop. However, if the slurry releases only a portion of the trapped gas, it would still be possible for the slurry to sink to the lower layers of the tank, provided that enough gas was released to increase the slurry density above that of the overlying fluid. Some justification for partial releases must be found before this theory may be deemed plausible; such a theory could, perhaps, involve partial layers of slurry reaching density instability at different times, while other layers remain stable. 


\subsection{EFFECT OF HYDROSTATIC PRESSURE VARIATION}

Epstein and Fauske, in an internal letter, submitted an analysis of the $R-1$ model in which it was concluded that the change in the slurry density due to the trapped gases was sufficient to cause the slurry to overturn. Their analysis included the effect of the hydrostatic pressure variation on the slurry density, which was neglected in the analysis performed above. However, their analysis suffered from inconsistent use of definitions for the volume fraction of gas and the porosity of the solids. A complete analysis of the effect of the pressure variation on the slurry density is presented here. The results of the analysis show that the effect of the hydrostatic pressure variation on estimates of the slurry density is negligible.

It is useful to define the void ratio, $\epsilon$, which describes the volume of gas trapped per volume of slurry:

$$
\epsilon=\frac{V_{g}}{V_{s}}
$$

where $V_{g}=$ the gas volume

$V_{s}=$ the slurry volume.

The void ratio can be related to the gas volume fraction, $\alpha$, which is defined as

$$
\alpha=\frac{V_{q}}{V_{g}+V_{s}}
$$

The relationship between $\alpha$ and $\epsilon$ is

$$
\epsilon=\frac{\alpha}{1-\alpha}
$$


and

$$
\alpha=\frac{\epsilon}{1+\epsilon}
$$

If it is assumed that the mass of gas trapped per unit volume of slurry, $m_{t}$, is a constant throughout the slurry layer, and that the gas density, $P_{g}$, varies with elevation, $z$, then $\epsilon$ varies as

$$
\epsilon(z)=\frac{m_{t}}{\rho_{g}(z)}=\frac{\text { mass gas/volume slurry }}{\text { mass gas/volume gas }}
$$

It is assumed that the local gas density varies according to the ideal gas law:

$$
P_{g}(z)=\frac{P_{g}(z)}{R_{g} T}
$$

where $P_{g}(z)=$ the pressure of the gas versus height

$R_{g}=$ the gas constant for the gas mixture

$T=$ the absolute temperature of the gas. The temperature varies throughout the slurry but will be assumed to be constant for this calculation.

This relationship may be written as

$$
\rho_{g}(z)=C_{g} \cdot P_{g}(z)
$$

where $C_{g}$ is a constant and is defined as

$$
c_{g}=\frac{1}{R_{g} \top}
$$


When the slurry is motionless, the pressure in the gas equals that of the liquid contained in the slurry. If the slurry solids are supported by the liquid, then the hydrostatic pressure gradient in the slurry must vary as

$$
\frac{d P_{s}}{d z}=\rho_{s} g
$$

This type of variation might be expected if the slurry liquid is capable of immobilizing particles and preventing them from touching each other. Some clay-water mixtures exhibit this type of packing; when dried, such mixtures exhibit shrinkage. Starr reports in an internal letter that the particles in some synthetic slurries are not in contact and that the slurries look like "sand in airplane glue." This suggests that it is possible that the particles in the fluid are not in contact with each other, and that the pressure in the fluid must be sufficient to support the entire weight of the slurry mixture.

If the slurry solids are not supported by the liquid, then the hydrostatic pressure gradient in the slurry varies as

$$
\frac{d \rho}{d z}=\rho_{1} g
$$

where $\rho_{1}$ is the liquid density.

This type of pressure variation would be expected in a settled bed in a sand-water mixture.

Either form of the pressure variation is physically possible for some types of slurries. It is known on the basis of Mauss's letter that the volume of centrifuged solids in the unexpanded lower layer of slurry is no more than $60 \%$. However, the centrifuge broke during testing, which may have prevented complete removal of the liquid in the slurry and reduced the ultimate level of packing of the solids. Reported volumes of centrifuged solids include both the volume of the solids at maximum packing and the volume occupied by the voids between solids. Since the maximum solids packing volume cannot reach one, the actual voiume of solids is always less than or equal to the centri-

\subsection{1}


fuged volume of solids. Thus the liquid void fraction is at least $40 \%$ and may be higher. A true solids fraction of $60 \%$ is in the range where either pressure variation is possible; however, lower solids fractions may suggest that the particles in the slurry are not in contact with each other.

It $w i 11$ be assumed here that the particles are not in contact and that the hydrostatic pressure varies as in Equation (6.16). Use of Equation (6.17) leads to predictions of greater stability since the pressure variation across the slurry layer is not as great. The first formulation was selected for analysis to show the maximum possible effect of the pressure variation on the variation in the slurry density. This results in a gas pressure that varies as

$$
P_{g}(z)=P_{b}-\int_{0}^{z} P_{s}(\zeta) g \zeta d \zeta
$$

where $P_{b}=$ the density at the bottom of the slurry

$$
\rho_{s}=\text { the slurry density }
$$

$\zeta=$ a dummy variable denoting the distance from the tank bottom.

The pressure at the bottom of the tank, $P_{b}$, may be determined using

$$
P_{b}=P_{a t m}+p_{1} g H_{2}(1-x) H_{2}+\rho_{s 2} g H_{2} x
$$

where $x=$ the fraction of the tank contents occupied by slurry

$$
\begin{aligned}
& \mathrm{H}_{2}=\text { the total height of the tank contents. These are shown in } \\
& \text { Figure 6.2. }
\end{aligned}
$$

Prior to expansion, the slurry density may be assumed to be constant, and the relationship between the gas pressure and the location, $\zeta$, in the slurry can be evaluated in a straightforward manner:

$$
P_{g}(z)=P_{b}-P_{s 2} g z
$$


After expansion, the slurry density varies with height, and the relationship is

$$
P_{g}(z)=P_{b}-\int_{0}^{z} \rho_{s 1}(\zeta) g \zeta d \zeta
$$

where the local density of the expanded slurry $\left[\rho_{s 1}(z)\right]$ is (density of unexpanded slurry times volume of unexpanded slurry)/(volume unexpanded slurry plus volume gas)

$$
\rho_{s 1}(z)=\rho_{S 2}[1-\alpha(z)]=\frac{\rho_{S 2}}{1+\epsilon(z)}
$$

It is significantly more difficult to evaluate the pressure variation in the unexpanded slurry than in the expanded slurry, since the slurry density, $P_{s 1}(z)$, is not a constant in this case, but is a function of the gas pressure, $P_{b}(z)$. The algebraic complexity of evaluating the pressure gradient in the expanded slurry may be avoided by determining the amount of gas that will be trapped in an unexpanded layer of slurry located between heights $z^{\prime}$ and $z^{\prime}+$ $d z^{\prime}$. After expansion, the total mass of gas trapped in the slurry layer, $M_{t}$, will be:

$$
M_{t}=m_{t} A_{t} d z^{\prime}
$$

After expansion, the layer of slurry that was at $\zeta^{\prime}$ will rise. However the hydrostatic pressure at the new location will be identical to the pressure prior to expansion since the pressure at the layer elevation must be sufficient to support the weight of the tank contents above the slurry both before and after expansion. If the total mass above the slurry layer does not change, and the pressure in the tank head space does not change, then the pressure exerted on the gas in this layer remains unchanged. It is thus not necessary to know either the new location at which the layer is foind or to know the new slurry density profile to determine the pressure at the layer. 
This implies that the gas trapped in this layer will occupy a volume, $V_{g}$, of

$$
V_{g}=\frac{m_{t} A_{t} d a^{\prime}}{\rho g(z)}=\frac{m_{t} A_{t} d z^{\prime}}{C_{g}\left(P_{b}-\rho s 2 g z^{\prime}\right)}
$$

The quantity $\epsilon^{\prime}\left(z^{\prime}\right)$ will be defined as the volume of gas trapped per unit volume of slurry that was originaliy at location $z^{\prime}$. The magnitude of this quantity is equal to

$$
\epsilon^{\prime}\left(z^{\prime}\right)=\frac{V_{g}}{V_{s}}=\frac{m_{t} / c_{q}}{\left(P_{b}-P_{s 2} g z^{\prime}\right)}
$$

The new height of the layer can be found by adding the unexpanded slurry volume and the additional gas volume and dividing by the tank area. This is equivalent to evaluating the following integral:

$$
z\left(z^{\prime}\right)=z^{\prime}+\int_{0}^{z^{\prime}} \epsilon^{\prime}(\zeta) d \zeta
$$

Thus, once the variation in $\epsilon^{\prime}$ is known, it is possible to determine the relationship between pressure and tank elevation. However, that will not be necessary for this analysis.

The new location of the top layer of the slurry may be determined using Equation (6.30) to evaluate the change in height of the top layer of slurry:

$$
\Delta h=\zeta\left(h_{s 2}\right)-h_{s 2}=\int_{0}^{h_{s 2}} \epsilon^{\prime}\left(z^{\prime}\right) d z^{\prime}
$$

Substituting in the relation for the void ratio, Equation (6.25), results in 


$$
\Delta h=\int_{0}^{h_{s 2}} \frac{m_{t} p_{b} c g}{\left(1-p_{s 2} g z^{\prime} / p_{b}\right)} d z^{\prime}
$$

Integration results in

$$
\Delta h=\frac{-m_{t} / C g}{\rho_{s 2} g} \ln \left(1-\rho_{s 2} g h_{s 2} / p_{b}\right)
$$

This equation may be used to determine the ratio of $\mathrm{m}_{\mathrm{t}} / \mathrm{C}_{\mathrm{g}}$.

Once $m_{t} / c_{g}$ is known, the void ratio, $\epsilon$, can be found by evaluating Equation (6.25), and the density of the expanded slurry that was initially at a location $z^{\prime}$ can be evaluated using Equation (6.22).

Equation (6.29) may be written in a form simflar to that obtained by Epstein and Fauske. (a) Using Equation (6.25) to substitute for $m_{t} / C_{g}$ results in

$$
\Delta h=\frac{\left(P_{b}-\rho_{s 2} g\right) \epsilon_{T}}{\rho_{s 2} g} \cdot \ln \left[\left(P_{b}-\rho_{s 2} g h_{s 2}\right) / P_{b}\right]
$$

where $\epsilon_{T}$ is the vold ratio at the top of the slurry. This is similar to the relation obtained by Epstein and Fauske. ${ }^{(a)}$

The value of the slurry density at the top and bottom of the slurry layer was evaluated using the values in Table 6.1. For the first case where the minimum unexpanded tank volume is assumed to be $403 \mathrm{in.}$, the specific gravity at the top of the slurry was found to be 1.62, while that at the bottom of the slurry was 1.64 . The stability ratio for the top layer was found to be $\sigma_{t}=\rho_{s 2 t} / \rho_{1}=1.219$, which is stable. The ratio found by neglecting the hydrostatic pressure variation was $\sigma=p_{\mathrm{s} 2} / p_{1}=1.225$. For the second case, where the unexpanded tank contents level was assumed to be $387 \mathrm{in}$., the

(a) M. Epstein and H. Fauske, letter to D. Stepnewski. 
ratto for the top layer was found to be 1.072 , whtch is also stable; the stability ratio found by neglecting the hydrostatic pressure vartation was 1.093 .

It is clear that the uncertainty in the unexpanded slurry height has a much more signiffcant effect on the estimated value of the stability ratio than the effect of the variation in the hydrostatic pressure. The $1 \mathrm{im}$ tations of this analysis are identical to those described for the previous analysis that did not account for the hydrostatic pressure vartation on the slurry density.

Finally, the effect of temperature vartation on the slurry density has not been investigated. It is posstble for the temperature vartation to amplify the slurry density variation. However, since the maximum temperature and mintmum pressure do not occur at the same tank elevation, it is unltkely that this occurs. In addition, the maximum and minimum temperatures in the lower slurry layer are $142^{\circ} \mathrm{F}$ and $127^{\circ} \mathrm{F}$, respectively. This would result in a maximum gas density difference of $2.5 \%$; the effect on the slurry density would be much smaller. Even a difference of $2.5 \%$ would not be suffictent to destabilize a slurry that had a stability ratto of 1.072 .

The stability analysis also was used to determine the expanded contents level at which level drops could be expected to occur. If the unexpanded level is assumed to be $403 \mathrm{in}$., which is equal to the lowest level recorded between 1981 and 1990, the slurry will become unstable when the contents reach a height of $440.9 \mathrm{in}$. If the unexpanded level of the contents is assumed to be $387 \mathrm{in.}$, as estimated by Simpson (letter), then the contents must reach $422.6 \mathrm{in}$. before the slurry becomes unstable. The maximum contents level recorded appears to be approximately $423 \mathrm{in}$. This occurred in 1989. A11 other level drops occurred at lower tank levels.

It appears unl tkely that the R-1 mechanism is causing the tank level drops unless one of the following conditions are true:

1. The specific gravity of the lower slurry is significantly less than 1.69 .

2. The specific gravity of the upper layer of flutd is significantly greater than 1.33. 
3. The regton in whtch gas is trapped occuptes less than one-third of the tank volume.

4. The unexpanded tank volume has declined significantly due efther to evaporation or leakage. (In this case the fact that the level of the tank contents after gas releases is not declining with time must be exp?atned.) 


\subsection{HYDROGEN BUBBLE FORMATION AND SIZE}

In contemplating the R-3 model (see Section 4.2.3), it is important to determine the size of the bubbles involved. The bubble size will deterintne the rate that they rise, the conductivity of the sludge mixture, and ultimately the viabllity of models that involve bubble dynamics.

This section discusses the following aspects of the bubble theory:

- Hydrogen and other gases form by a steady chemtcal reaction in the waste sludge.

- The gases butld to a saturated condition.

- Clusters of gas form at a multitude of nucleation stes that are in the sludge due to tts high heterogeneous solid content.

- Gases continue to form and diffuse to the local cluster of gas molecules causing the cluster to grow rather than activating a new nucleation site.

Therefore, the number of bubbles formed per untt volume depends on the diffustutty of the gas and on the rate of gas formation.

If the diffusion is high enough at the subsaturated concentration to allow the gas production to be diffused to a bubble nearby, then no new bubbles will form and the extsting bubbles will simply grow. Perhaps, according to R-3 model (Section 4.0), when they get large enough these bubbles begin to rise in the sludge and trigger a scavenging action that releases many of the other bubbles that should have also grown to about the same size. The waste collapses down and mixes due to the bubble release.

Consider a single gas, such as hydrogen. As shown in Figure 7.1, the concentration builds up to $C_{\text {sat }}\left(C_{0}\right)$ at which nucleation and bubble growth begin at sites such as site 1 and site 2. Other nearby sites such as site 3 did not reach nucleation and were robbed of the nearby dissolved gas by the diffusion to the nucleated sites (1 and 2). Further production of gas in the region between sites 1 and 2 diffuses to them also. The steady-state condition is achieved when the rate of gas formation in a spherical shell or radius $R$ is diffused by the concentration gradient to the spherical bubble surface. 


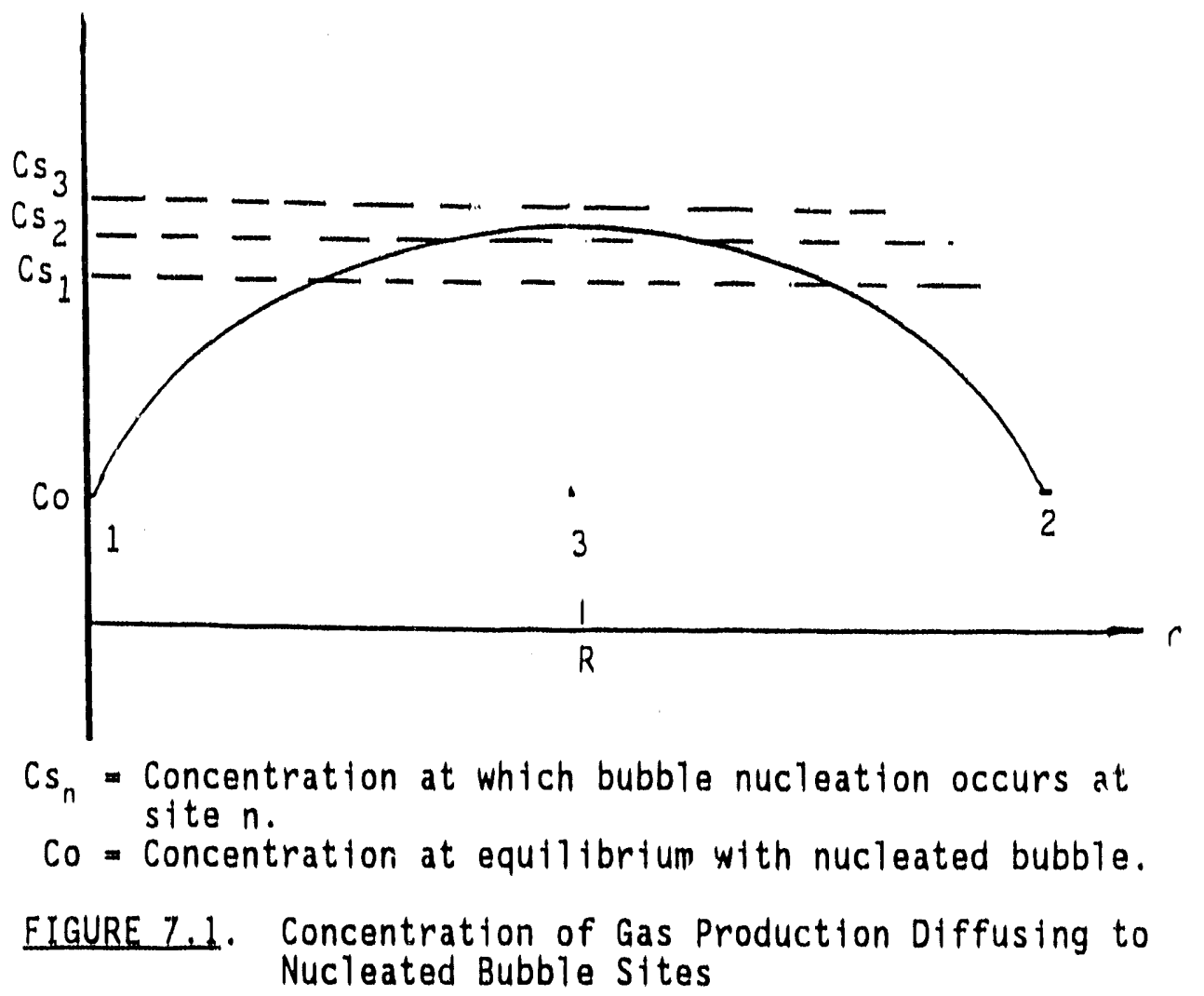

Only the initial nucleated sites grow into bubbles because bubble growth reduces the concentration in the vicinity. As the bubble grows, the sphertcal shell is displaced outwards. It contains the same total volume of substance that is generating the gas, but the diffusion distance is reduced. This increases the gradient and reduces the average concentration. As the bubbles grow, the area through which diffusion takes place to reach the bubble surface also increases and will cause a decrease of the concentration. This effect is larger than that of the decreased diffusion distance. The combined effect is even larger, as shown in Figure 7.1. The reduced concentration caused by the ever-increasing ease of diffuston to the bubbles already nucleated reduces the tendency for more nucleation sites to be activated and promotes the continued growth of the already existing bubbles. Therefore, the number of bubbles created depends on the diffusivity of the created gas in the waste matrix as well as on the rate of creation. 
The heterogenous nature of the waste sludge should provide nucleation sites in abundance. If not, homogeneous nucleation could a'so occur, but it may involve a supersaturated condition. As an illustration of nucleation, supersaturated water can be made to nucleate by introducing a boiling chip or impurity with nucleation sites; a glass of beer will foam more rapidly if salt is sprinkled into it.

At the beginning of nucleation of a bubble, the cluster of gas molecules would be smaller than the mean free path of the dissolved gas. Therefore, the rate of growth will be a function of mean free path. However, the situation that determines the number concentration of growing bubbles occurs later when the bubble is larger and the growth does not depend on random molecular collisions but on the rate of diffusion of the gas molecules to the bubble surface. The situation is shown in Figure 7.1.

The concentration of gas that is being formed initially in solution builds up to a supersaturated concentration, $C_{s 1}$, at which a bubble forms at site 1 . This hubble allows the concentration at the surface to drop to some equilibrium value, Co, setting up a concentration gradient toward the bubble. Beyond the gradient, dissolved gas continues to raise the concentration such that another site (such as site 2) begins to form a bubble also. The dissolved gas also begins to diffuse to the bubble at site 2 , and the concentration continues to rise in places remote from the formed bubbles. However, before the concentration rises to a level where site 3 would form a bubble, the diffusion of the gas toward the bubbles already formed takes all the gas being formed and the dissolved concentration ceases to rise. The concentration holds steady or falls so that no new bubbles are formed at sites such as site 3 . The half distance between bubbles formed is $R$ and is in some way dependent on the rate of gas formation and the diffusivity of the dissolved gas. The actual concentration will depend also on the size of the bubble for reasons discussed in Appendix $A$.

Appendix $B$ is an analysis that gives the bubble distance at nucleation cutoff, which can allow the number density of bubbles to be calculated from some physical parameters. Appendix $C$ is a derivation of a set of trial parameters. 
As shown in Appendix $B$, an estimate of the minimum bubble size that can grow will give a number concentration of those bubbles in the collapsed waste. For the example parameters used, a $10^{-5} \mathrm{~cm}$ bubble will have a concentration of about $1 / \mathrm{mL}$. With a gas production rate of $0.3 \mathrm{~cm}^{3} / \mathrm{L} /$ day and an 8.5 -week cycle time, the bubbles when released would be $0.32 \mathrm{~cm}$ in diameter.

The actual volume of gas given off in a cycle will then give the typical volume (size) of the bubble (assuming uniform bubble size) that has left the sludge during the collapse. This size can be used to aid or corroborate theories dealing with the movement of the bubbles through the sludge. Items such as bubble rise velocity and shear stress resistance to movement depend on the size of the bubble.

Further development of this theory might take the following into account:

- the effects of sludge viscosity and composition on the diffusion coefficient

- the effect of the production of other gases and the combined gas diffusion and bubble growth

- the effect of hydrostatic head on the bubble growth and diffusion parameters

- the effect of temperature in the sludge on the parameters

- a combination of the theory with bubble dynamics, i.e., does bubble motion influence the diffusion pattern and growth?

- a consideration of the effects combined, i.e., which bubbles start to rise and how do they trigger the rest? 


\subsection{DESCRIPTIVE MODEL FOR BUBBLE SIZE AND MOTION}

The existence of a solid crust over a relatively fluid slurry and a viscuus sludge (slurry) at the bottom of the double-sheli Tank 101-SY has resulted in several different theories of the waste behavior within the tank. This section describes some details of the R-3 model (Section 4.2.3), one possible theory of how the slurry-generated jases may be transported through the sludge and the liquid above it before they encounter the crust. The process of the gases penetrating through the crust and getting released into the tank air space and to the outside through the vent is the last step in a gas release event (a "burp" of the tank). The production and distribution of the gases (predominantly hydrogen and nitrous oxide) within the tank are assumed to be uniform throughout the sludge and slurry volume. They would initially form very small bubbles and be dispersed so that they remain small and essentially stationary.

Several experimental observations of the shapes of the gas bubbles in different pseudoplastic and thixotropic (non-Newtonian) liquids have shown that sıveral transformations occur. The bubble shapes would change from spherical, to prolate spheroidal, to that of a top (this transition occurring at a critical bubble size, which will be discussed later), to that of an ablate spheroid with a tail, and finally to that of a spherical cap (Calderbank et al. 1970). Bubbles in the aqueous Polyox (polyethylene oxide) solution do not exhibit surface rippling and rise rectilinearly without rocking or pulsation, unlike in Newtonian media. Bubble surface areas also tend to be generally lower compared to those in distilled water (Newtonian fluids). Because of the density difference between the gas and the slurry, the bubbles rise through the slurry medium (considered as a non-Newtonian medium) only after they grow to a certain size and are able to overcome the viscous drag force imposed on them.

A detailed description of the different size ranges (bubble radius, $R_{b}$ ) of the bubbles and their rise velocities (U) follows. 


\section{$8.1 \underline{\mathrm{R}}_{\mathrm{b}} \leq 10 \mu \mathrm{m}$}

The bubbles are just forming from the dispersed phase of the slurry liquid and are still so small that they remain stagnant at the nucleation sites and well distributed throughout the medium.

\section{$8.210 \mu \mathrm{m}<\mathrm{R}_{\mathrm{b}} \leq 50 \mu \mathrm{m}$}

In this size range, the bubbles begin to move or drift very slowly ( $U$ [velocity of bubbles] $=-0.005 \mathrm{~cm} / \mathrm{s}$ ) in the slurry medium. They essentially behave as rigid spheres with an increased viscosity in a thin but microscopic layer near the liquid interface. The "viscoelastic" nature of the slurry and the nature and degree of surfactants present at the bubble surface also contribute to the reduced mass transfer coefficient of the gases.

\section{$8.350 \mu \mathrm{m}<R_{b} \leq 100 \mu \mathrm{m}$}

This is a likely size and velocity range for most of the gas bubbles formed in the slurry and trapped deep within the tank. They also remain in this state for a long period of time before the reaction kinetics give rise to larger volumes of the slurry gases. The velocity begins to increase to about $0.01 \mathrm{~cm} / \mathrm{s}$, but the corresponding Reynolds number still remains quite small $\left(-10^{-6}\right)$ and the associated drag coefficient quite large $\left(-10^{6}\right)$. The rigid sphere motion of these bubbles yields to the classical Stokes solution regardless of whether the surrounding medium is treated as Newtonian or non-Newtonian.

\section{$8.4 \underline{100 \mu \mathrm{m}<R_{b} \leq 500 \mu \mathrm{m}}$}

As more gas is formed due to either a build-up of the temperature or a higher reactivity of the slurry, the bubble volume grows to the order of $10^{-4} \mathrm{~cm}^{3}$ and the bubbles pick up some speed. The rise velocity becomes of the order of $0.1 \mathrm{~cm} / \mathrm{s}$ and the bubbles start encountering other bubbles and may coalesce to form even larger bubbles. As they move up, they also expand due to the lower hydrostatic pressure at higher elevations, and the liquid envelope around these bubbles would become thinner and weaker. At these rise velocities, it would take only about $1.5 \mathrm{~h}$ for the bubbles to traverse the 
$18 \mathrm{ft}$ or so of the slurry medium to reach the liquid present above the sludge. However, it is the gas dispersion and the low fraction of the gas volume (or "gas holdup" in the tank) that keeps most of the bubbles well below the $100-\mu \mathrm{m}$ size for an extended period of time.

\section{$8.5 \underline{500 \mu \mathrm{m}<R_{b} \leq 2000 \mu \mathrm{m}(0.2 \mathrm{~cm})}$}

The bubble volume becomes $10^{-3}$ to $10^{-2} \mathrm{~cm}^{3}$ and the rise velocity increases to about $0.5 \mathrm{~cm} / \mathrm{s}$. At this stage, some of the gas bubbles have already risen to the top of the liquid and are gathered below the crust (the level of the crust has gone up by now) still in the form of discrete bubbles and not as a large gas pocket or layer. The off-gas release at this point is imminent and the bubbles that are still trapped in the slurry will now be able to move quite rapidly to the crust (in less than $-30 \mathrm{~min}$ ). The situation of the gas bubbles trapped below the crust, however, could not last very long before the gas is released and the crust surface level drops. The actual gas release mechanism is uncertain, but it could penetrate either through cracks in the crust surface or between the crust and metal surfaces such as tank walls and/ or pipes that have been inserted through the crust.

\section{$8.60 .2 \mathrm{~cm}<R_{b} \leq 0.3 \mathrm{~cm}$}

In this bubble radius range, the bubble motion undergoes a velocity jump to approximately $2.0 \mathrm{~cm} / \mathrm{s}$ at a certain critical radius $(\sim 0.21 \mathrm{~cm})$ and a $\mathrm{flow}$ field transformation from a "rigid sphere" motion to a "fluid sphere" (with circulation) motion. This transformation corresponds to the transition from the Stokes regime to the Hadamard-Rybczynski regime in Newtonian fluids, and also results in a sharp reduction in the drag coefficient (a "shear-thinning" of the surrounding medium), and the bubbles begin to rise much faster (they can traverse the distance of approximately $34 \mathrm{ft}$ to the crust in less than 10 min). Therefore, even if some of these larger gas bubbles are trapped below during most of this time (by solid particulates or chunks of material), a "triggering" mechanism such as a reduction in pressure or a greater tendency toward coalescence duf: to the penetrated (and eventually falling) crust will enable them to get untrapped and rise through the tank rapidly. The 
subsequent release of the gases to the tank air space and through the vent will occur in the few minutes immediately following a major off-gas release. This could cause the crust to fall even further and consolidate itself until the remaining slurry liquid envelope is sufficiently strong to contain the gas bubbles. 


\subsection{DISCUSSION OF VOLUME AND LOCATION OF GAS}

There are three factors that are observed during an off-gas release event, namely, the crust level drop, the pressure build-up in the tank airspace, and the increase in the volume flow rate through the vent. An attempt is made here to quantify the volume and the possible state (location and pressure) of the gas before and during the off-gas release event based on these three factors.

\subsection{CRUST LEVEL DROP AND PRESSURE RISE}

The first step of the analysis is based on the crust level drop and the pressure build-up between two states of the tank airspace: the instant before the crust level starts dropping and the instant after it has dropped the total amount defining the event. Let us assume that the state before the event is represented by pressure, $P_{0}$, temperature, $T_{0}$, volume, $V_{0}$, and mass, $m_{0}$, and the state after the event by $P_{1}, T_{1}, V_{1}$, and $m_{1}$, respectively. The change in the conditions of the state is only because the slurry growth gases flowed into the tank airspace from below the crust while the total temperature $\left(T_{0}\right)$ remained constant ( 90 to $100^{\circ} \mathrm{F}$ or 550 to $560 \mathrm{R}$ ).

Based on an ideal gas assumption, the change in mass of gas (assumed to have a molecular weight of 28 ) in the tank airspace is given by:

$$
\Delta m=m_{1}-m_{0}=\frac{\left(P_{0}+\Delta P\right)\left(V_{0}+\Delta V\right)}{R T_{0}} \quad \frac{\left(P_{0} V_{0}\right)}{R T_{0}}
$$

where $P_{1}$ and $V_{1}$ have been substituted as $\left(P_{0}+\Delta P\right)$ and $\left(V_{0}+\Delta V\right)$, respectively. $\Delta P$ corresponds to the $+5.2-$ in. water gage pressure rise in the tanks $(-2.9$ in. to $+2.3 \mathrm{in}$. water gage), while $\Delta V$ corresponds to the $+9.2-i n$. crust level drop. Hence, we get

$$
\Delta \mathrm{m}=341.6 \mathrm{lbm}
$$

and the volume of the gas corresponding to $\Delta \mathrm{m}$ is $4379.5 \mathrm{ft}^{3}$. 
If we now consider the crust level drop to be from 413.2 in. to $402.9 \mathrm{in}$. (10.3 in.) including the sharp crust level rise before the event started, then the total mass released would be $371.6 \mathrm{lbm}$, and the corresponding volume would be $4764 \mathrm{ft}^{3}$.

Now the data show that in the changing situation when the total crust level drop occurs, the pressure in the airspace is clearly not $+2.3-i n$. water gage but much lower. So the gas volumes calculated above represent an upper bound on what could be predicted from purely an ideal gas behavior and two distinct states of the gas point of view.

\subsection{INCREASE IN VENT FLOW RATE}

If we now consider the air inleakage and the fact that the vent flow rate increased from $611 \mathrm{cfm}$ (prior to the event) to about $2000 \mathrm{cfm}$ at the peak, and then back down to $611 \mathrm{cfm}$ after the first $5 \mathrm{~min}$, then the rate of inflow of slurry growth gas would be $\dot{m}_{\mathfrak{i}}$ when the accumulation rate of gas in the header volume (tank airspace) would be $(\mathrm{dm} / \mathrm{dt})_{\mathrm{cr}}$, or $\Delta \mathrm{m}$ calculated above, during the 5 -min event.

Therefore, the dynamic mass balance yields

$$
(\mathrm{dm} / \mathrm{dt})_{c V}=\Sigma \dot{m}_{i}-\Sigma \dot{m}_{\mathrm{e}}
$$

or

$$
\Delta m=\Sigma\left(\dot{m}_{i}-\dot{m}_{e}\right) \cdot \Delta t
$$

Here $m_{e}$ is determined from the net outflow through the vent $(2000 \mathrm{cfm}$ to $611 \mathrm{cfm})$ plus the net outflow of gases from the tanks to the outside because of the reversal in the pressures in the tank header volumes. If the pressures in tanks 102-SY and 103-SY went up only to +0.8-in. water gage with an air outleakage of about $50 \mathrm{cfm}$, as opposed to a pressure rise in tank 101-SY to $+2.3-\mathrm{in}$. water gage with an outleakage of approximately $200 \mathrm{cfm}$, then the total outflow of mass is at the rate of $m_{e}=589+50+50+200=889 \mathrm{cfm}$. 
Therefore, we get $m_{f}=\left(371.61 \mathrm{bm}+889 \mathrm{cfm} \cdot 0.0781 \mathrm{bm} / \mathrm{ft}^{3} \cdot 5 \mathrm{~min}\right) /$ $5 \mathrm{~min}$ and,

$$
\dot{\mathrm{m}}=143.66 \mathrm{lbm} / \mathrm{min}
$$

The total volume of gases released from under the crust is that accumulated in the head space plus that vented during the increased outflow of gases because of the pressure build-up.

$$
V_{i}=\left(\dot{m}_{1} \cdot \Delta t\right) / 0.0781 \mathrm{bm} / \mathrm{ft}^{3}=6446 \mathrm{ft}^{3} \text { in the first } 3.5 \mathrm{~min}
$$

On the basis of the FATHOMS modeling, a Westinghouse Hanford Company employee ${ }^{(a)}$ calculated the total volume of gases released as approximately $7000 \mathrm{ft}^{3}$ in the first $3.5 \mathrm{~min}$. Therefore, even this very simplified bruteforce analysis gives a good approximation of the total volume of the gases.

\subsection{LOCATION AND STATE OF THE GASES}

The larger gas volume that was calculated (compared to the volume corresponding to the crust level drop of $9.2 \mathrm{in}$., which is only $3387 \mathrm{ft}^{3}$ ) would imply that the gas remained compressed, if not dissolved in the slurry, below the crust. The pressure ratio is given by

$$
\begin{aligned}
P_{1} / P_{2}=V_{2} / V_{1} \cdot T_{1} / T_{2} & =6446 / 3387 \cdot(460+120) /(460+90) \\
& =2.007
\end{aligned}
$$

The hydrostatic pressure to give this ratio is at a depth " $h$ " such that

$$
P_{1}=2.007 P_{2}=P_{2}+\rho g h
$$

(a) T. Burk, Westinghouse Hanford Company, private communication. 
and $P_{2}=14.58$ psta in the atrspace (corresponding to the $-2.9-\mathrm{tn}$. water gage condition).

With an assumed value for specific gravity of the slurry as 1.6 , we get

$$
h=[(2.007-1) \cdot 14.58 \cdot 144] /(1.6 \cdot 62.4)=21.1 \mathrm{ft}
$$

which is just about $12 \mathrm{ft}$ off the bottom. This is well within the slurry medium. If the assumed specific gravity of the slurry were any lower, the compressed gas would be even deeper, and if it yields a value greater than the depth of the tank, it would requite that some of the gas released is not compressed, but remains dissolved in the slurry medium prior to the event. For example, some of the nitrous oxide $\left(\mathrm{N}_{2} \mathrm{O}\right)$ gas may remain dissolved in the slurry, while the hydrogen gas is in the form of bubbles and is released first during the off-gas release event. 


\subsection{CURRENT PLANS}

Data analysis is continuing, and we are accessing a larger data base which increases our understanding.

Workshop discussions with staff from Westinghouse Hanford Company who are familiar with the tank data and history will help adjust and eventually support the models as they are developed and will suggest what new data are needed. The tasks of modeling development are exploratory at this time and may not answer all objections or explain all the data. On the other hand, the models that are studied under this proposal may be successful in eliminating some of the several mechanisms or theories that have been suggested.

One of the largest obstacles has to do with obtaining good data from the tank. The physical properties of the liquid and slurry under the crust are very important data. Those samples that were taken in 1987 have given the only real data that we have, and they are inconsistent. In the absence of better data, much effort has to be spent on resolving the inconsistencies. Modeling hypotheses can be developed to some extent and await resolution by obtaining better information in the future. 


\subsection{BEFERENCES}

Armstrong, R. C., and H. H. Winter. 1983. "Heat Transfer for Non-Newtonian Fluids." Chapter 2.5.12 in Heat Exchanger Destan Handbook, E. U. Schlunder editor-in-chtef, Hemisphere Publishing Corp., Washington, D.C.

Bauer, R. 1983. "Stagnant Packed Beds." Chapter 2.8.1 in Heat Exchanger Design Handbook, E. U. Schlunder editor-in-chief, Hemisphere Publishing Corp., Washington, D.C.

Brennan, P. J., and E. J. Kroliczek. 1979. Heat Plpe Desian Handbook, pp. 47, 158. B \& K Engineering, Inc., Towson, Maryland.

Birke, T. M. 1990. Fathoms Analys is of Double-She11 Waste Tank SY-101 Gas Release (April 19, 1990 event). WHC-SD-WM-ER-067, West Inghouse Hanford Company, Richland, Washington.

Calderbank, P. H., D.S.L. Johnson, and J. Loudon. 1970. "Mechanics and Mass Transfer of Single Rubbles in Free Rise Through Some Newtonian and NonNewtonian Liquids." Chem. Eng. Sct. 25:235-256.

Cheng, H. C., and T. E. Natáii, Jr. 1986. "Measurement and Physical

Properties of Foam." Chapter 1 in Encyclopedia of Fluid Mechantcs, Volume 3, Cheremisinoff, ed., pp. 4-10, Gulf Publishing Co., Houston, Texas.

Cusster, E. L. 1984. Diffuston-Mass Transfer in Fluid Systems, pp. 185-187. Cambridge Univer'sity Press, New York.

Delegard, C. 1980. "Laboratory Studies of Complexed Waste Slurry Volume Growth in Tank 241-SY-101." RHO-LD-124, Informal Report. Rockwell Hanford Operations, Richland, Washington.

Gnielinski, V. 1983. "Fixed Beds." Chapter 2.5.4 in Heat Exchanger Design Handbook, E. U. Schlunder editor-in-chief, Hemisphere Publishing Corp., Washington, D.C.

Goldstein, R. J., and K. Kudo. 1982. "Convection in a Horizontal Fluid Layer Having a Shear-Free Upper Surface and Uniform Volumetric Energy Sources." Heat Transfer of 1982, Vol. II, pp. 129-134. In Proceedings of the 7th International Heat Transfer Conference, Hemisphere Pubitishing Corp., Washington, D.C.

Jansky, M. T. 1982. "Control Experiment for Slurry Growth: $80^{\circ} \mathrm{C} . "$ Proceedings on Waste Management, R. G. Post, ed.

Jansky, M. T. 1985. "Slurry Growth: The Characterization of a Unique Phenomenon at the Hanford Site." Waste Management '85, Vo1. 2 pp. 593-595. Kuriti, D., and J. M. Smith. 1960. "Heat Transfer Characteristics of Porous Rocks." AIChE J. 6(1):71. 
Maddox, R. N. 1983. "Thermophysical Properties." Chapter 5.2.3 in Heat Exchanger Destan Handbook, E. U. Schlunder editor-in-chtef, pp. 5.2.3-55.2.3-6. Hemisphere Publishing Corp., Washington, D.C.

Martin, H. 1987. "Flutdized Beds." Chapter 2.8.4 in Heat Exchanger Design Handbook, E. U. Schlunder editor-in-chtef, Hemisphere Publishing Corp., Washington, D.C.

Nielson, K. K., V. C. Rogers, and G. W. Gee. 1984. "Diffusion of Radon Through Solls: A Pore Distribution Mode1." Soll Sct. Soc. Am. J. 48(3):486.

Panjunen, A. L., S. M. Joyce, K. G. Carothers, and T. M. Burke. 1990. "Evaluation of April 19, 1990 Tank 241-SY-101 Gas Release Event." WHC-SD-WMPE-039., Westinghouse Hanford Company, Richland, Washington.

Ratcliffe, E. H. 1968. "Thermal Conductivities of Two-Phase Media: Methodology, Results, Estimations." Thermal Conductivity, pp. 1141-1147. Proceedings of the 8th International Conference. Plenum Press, New York.

Roark, R. J., and W. C. Young. 1975. Formulas for Stress and Strain, 5th Edition, McGraw-Hill, New York.

Schunck, M. 1983. "Thermal Conductivity of Solids." Chapter 5.4.3 in Heat Exchanger Destgn Handbook, E. U. Schlunder editor-in-chief, Hemisphere Publishing Corp., Washington, D.C.

Shulman, Z. P. 1983. Disperse Compositions. Chapter 5.3.1 in Heat Exchanger Design Handbook, E. U. Schlunder editor-in-chief, pp. 5.3.1-1 - 2 . Hemisphere Publishing Corp., Washington, D.C.

Timoshenko, S., and S. Woinowsky-Krieger. 1959. Thenry of Plates and Shells. Mcgraw-Hi11, New York.

Tsao, G. T. 1961. "Thermal Conductivity of Two-Phase Materials." J. Ind. Eng. Chem. 53(5):395.

Tsederberg, N. V. 1965. Thermal Conductivity of Gases and Liquids, p. 231. The M.I.T. Press, Cambridge, Massachusetts.

Ungan, A., and R. Viskanta. 1985. "Effect of Air Cubbling on Circulation and Heat Transfer in. a Glass Melting Tank." In Heat Transfer - Denver 1985, N. M. Farukhi, editor, pp. 70-83. AIChE, New York.

Viskanta, R. 1985. "Heat Transfer in Glass," in Heat Transfer - Denver 1985, N. M. Farukhi, editor, pp. 63-69. AIChE, New York.

Weast, R. C. (editor-in-chief). 1384. CRC Handbook of Chemistry and Physics, p. F-37. CRC Press, Inc., Boca Raton, Florida.

Weyl, W. A., and W. C. Ormsby. 1960. "Atomistic Approach to the Rheology of Sand-Water and Clay-Water Mixtures." Chapter 7 in Rheology, Vol. 3, ed. F. R. Eirich, pp. 249-297. Academic Press, New York. 
Zabrodsky, S. S, 1983. Fluid-to-Parttcle Heat Transfer in Fluidl\%ed Beds. Chapter 2.5.5 in Heat Exchanger Destan Handbook, E. U. Schlunder editor-inchtef, Hemlsphere Publishing Corp., Washington, D.C. 


\section{APPENDIX A}

\section{EFFECT OF DILATION ON DIFFUSION TO BUBBLES IN MATRIX}


APPENDIX A

\section{EFFECT OF DILATION ON DIFFUSION TO BUBBLES IN MATRIX}

Nucleated gas bubbles are situated in a matrix of waste material or sludge medium that produces the gas. Assuming that the gas production rate is steady in the medium, the gas bubbles grow by gas diffusion to them. The sludge mass consists of the expanding bubbles and the constant density medium. The sludge dilates, that is, it expands due to the growth of the bubbles. (The medium is assumed to remain of constant density although some mass [as gas] is being lost from it.)

We assume that the nuclei and subsequent bubbles are arranged in a spacefilling close-packed array such as hexagonal close-packed or face-centered cubic (FCC) array.

With the FCC cube of side length, 1c, the distance between space-filling spheres is $\sqrt{2} / 21 c$, which is equal to the maximum sphere radius.

The volume of the cube is $1 c^{3}$.

The volume of the spheres in the cube is

$$
\frac{6}{2} \cdot \frac{1}{6} D^{3}+8 \cdot \frac{1}{8} \cdot \frac{1}{6} D^{3}=\frac{2}{3} D^{3}
$$

The expansion of the bubbles as they collect gas makes the cube larger, but the medium volume stays the same at $1_{0}^{3}$.

The dilated cube has a side length of $1_{0}+D$, and a total volume of $\left(1_{0}+\right.$ D) ${ }^{3}$, or the volume $=$ bubble volume + volume of medium.

$$
1 c^{3}=10^{3}+2 / 3\left(D^{3}\right)
$$

The diffusion distance between bubble surfaces is proportional to the diagonal FCC cube distance $(\sqrt{2} / 2$ lc) minus the bubble diameter, $D$. 
So the diffusion distance $x=\sqrt{2} / 2 \cdot 1 c-D$, and a normalized diffusion distance,

$$
x / 1_{0}=\sqrt{2} 1 c / 21_{0}-0 / 1_{0}
$$

The diffusion rate $=D f d C / d x A$, where $D f=$ the diffusivity, $C=$ concentration, and $A=$ area. Diffusion rate is proportional to $A / x$, which normalizes to:

$$
A / x-A / 1_{0}^{2} / x / 1_{0}=\frac{4 \pi D^{2} / 1_{0}^{2}}{\sqrt{\overline{2} 1 C} / 21_{0}-D / 21_{0}}
$$

This equation has been plotted in Figure $A .1$ using an algorithm to make the calculations. This shows that $A / x$ increases as the bubble size increases, hence the diffusion to the bubble becomes easier and the concentration will fall more rapidly around the bubble and further preclude new bubbles from forming. Therefore, the growth reduces the nearby concentration of gas and increases the concentration gradient to further the diffusion toward the bubble. The growth of the early bubble works against the formation of new bubbles, such that those that are first formed will be the ones that grow to maximum size.

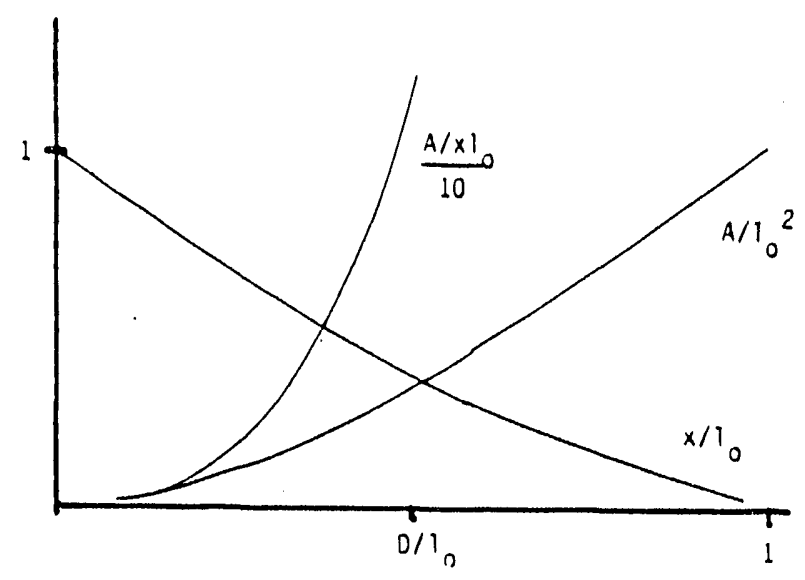

FIGURE A.1. Effect of Bubble Size on Diffusion Rate Factors 


\section{APPENDIX B}

\section{CALCULATION OF BUBBLE NUMBER DENSITY}




\section{APPENDIX B}

\section{CALCULATION OF BUBBLE NUMBER DENSITY}

We have assumed that there is a steady-state situation where gas production equals the rate of diffusion into a bubble formed and growing at a nucleation site.

$$
\text { Gas diffusion flux }=J=-D f d C / d r
$$

where $J=f 1 u \times M / L^{2}$

$$
\begin{aligned}
D f & =\text { diffusivity } L^{2} / t \\
C & =\text { concentration } M / L^{3} \\
F & =\text { gas formation rate in medium } M / L^{3} t .
\end{aligned}
$$

As in Figure B.1, the rate of collection in the nucleated bubble must equal the rate of production out to a radius where the diffusion begins to go toward another bubble (at radius $R$ ) because the concentration gradient $d C / d r=0$.

Production in shell of radius $R$ equals diffusion rate into the bubble at the bubble boundary.

$$
\int_{r}^{R} F 4 \pi r^{2} d r-D f \frac{d C}{d r} 4 \pi r^{2}=0
$$

Therefore,

$$
\frac{R^{3}}{r^{2}}-r=\frac{3 D_{f}}{F} \frac{d C}{d r} \text {. }
$$




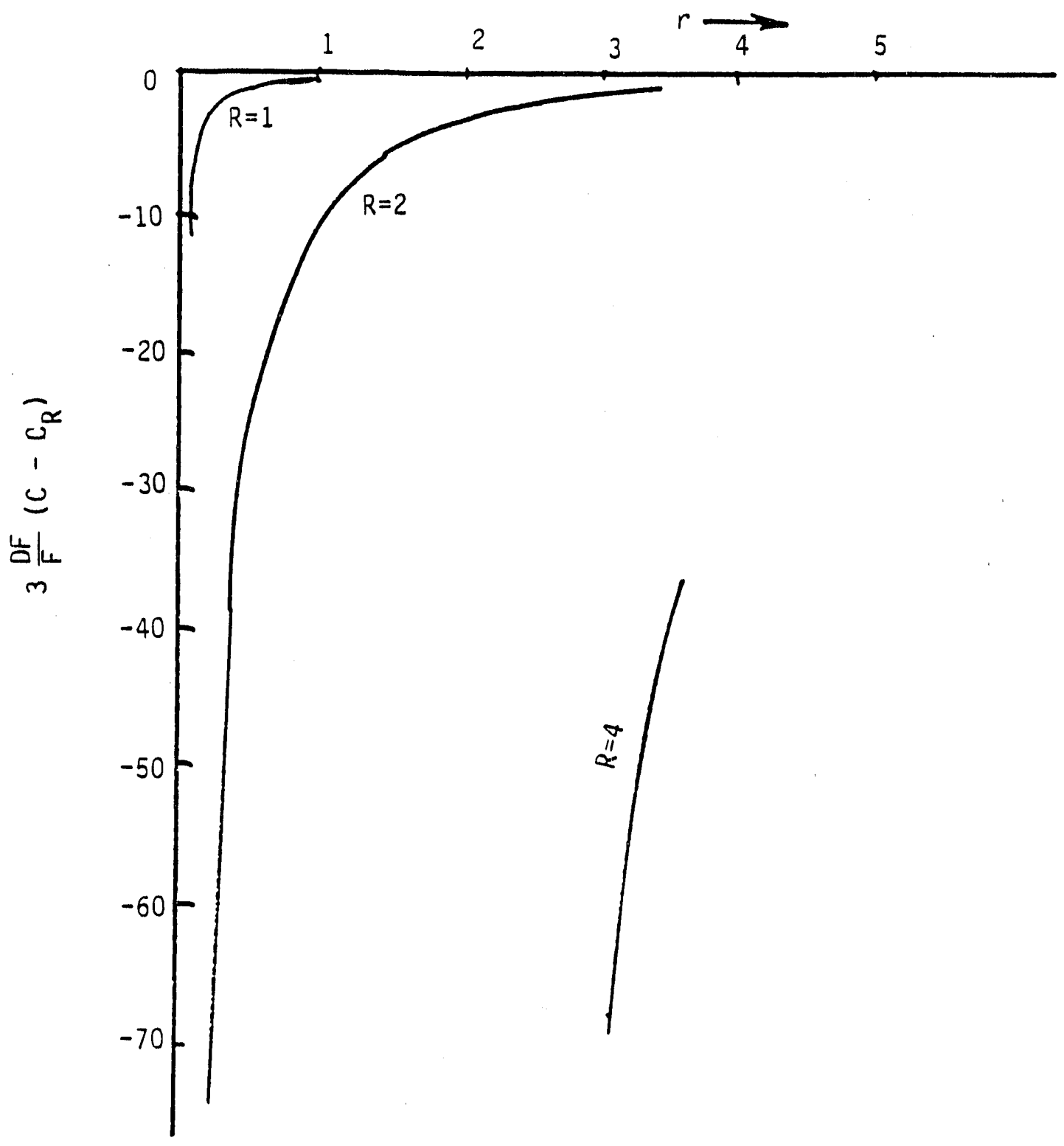

FIGURE B.1. Sample Calculation of Concentration Versus Distance from Bubble

Integrating from $r$ to $R$,

$$
C=C_{R}-\frac{F}{3 D_{f}}\left(\frac{R^{3}}{r}+\frac{r^{2}}{2}-\frac{3}{2} R^{2}\right)
$$

This equation was solved with a computer algorithm; the results are plotted as Figure B.1. Note that Equation (B.4) is a formula for 
concentration $C$ versus $r$, which depends on the parameter $R$. It seems possible that the physical constants of the case may determine what the value of $R$ has to be for the steady-state condition to hold.

The size of the bubble at the time of establishment of the steady-state concentration profile is limited by the adjacent bubble and will contain the gas that has formed and diffused to the bubble. That is,

$$
\frac{4 \pi}{B} r b^{3} \rho=\frac{4 \pi}{3} C_{R}\left(R^{3}-r b^{3}\right)-\int_{r b}^{R} C 4 \pi r^{2} d r
$$

also, from Equation (B.4),

$$
\left.C_{R}=C e+\frac{a}{3} \mid \frac{R^{3}}{r_{b}}+\frac{r_{b}^{2}}{2}-\frac{3}{2} R^{2}\right)
$$

where $C_{R}=$ concentration at $R$ the bubble half spacing, $M / L^{3}$

$$
\begin{aligned}
\rho & =\text { density of gas in bubble } M / L \\
a & =F / D f M / L^{5} \\
C e & =\text { the concentration at bubble surface } M / L^{3} .
\end{aligned}
$$

Substituting into Equation (B.5), simplifying, and integrating results in:

$$
r b^{3} p=a\left(\frac{R^{5}}{10}-\frac{R^{3} r b^{2}}{2}-\frac{r b^{5}}{10}+\frac{R^{2} r b^{3}}{2}\right)
$$

The physical constants that can be independently determined (estimated) are F, Df, Ce (see Appendix C).

Rearranging Equation (B.7) gives

$$
R^{5}=\frac{10 r b^{3}}{a} p+5 R^{3} r b^{2}-r b^{2}+5 R^{2} r b^{3}
$$


that can be solved by progressive trial and error. This has been done with a computer calculation routine. The results can be plotted as in Figure $B .2$ showing the spacing radius $R$ versus the minimum bubble radius. The ratio $R / r b$ is also shown.

The knowledge or estimate of the minimum bubble size that grows will therefore give the spacing of the bubbles. The number concentration of the bubbles at close-packed distance is four bubbles in a cube of $2 R / \sqrt{2}$ on a side. With a volume of $(2 R / \sqrt{2})^{3}$, the number density (concentration) is $\sqrt{2} / R^{3}$. For the parameters chosen, the graph of Figure B.2 gives

$$
\underset{(\mathrm{cm})}{R}=10.741 \underset{(\mathrm{cm})}{(\mathrm{rb})^{0.2}}
$$

Equation (B.9) can be used to derive an equation for the number density as a function of bubble radius, $r b$.

$$
\underset{(n u m b / c c)}{N}=1.14 E-3 \underset{(\mathrm{cm})}{(\mathrm{rb})^{-0.6}}
$$




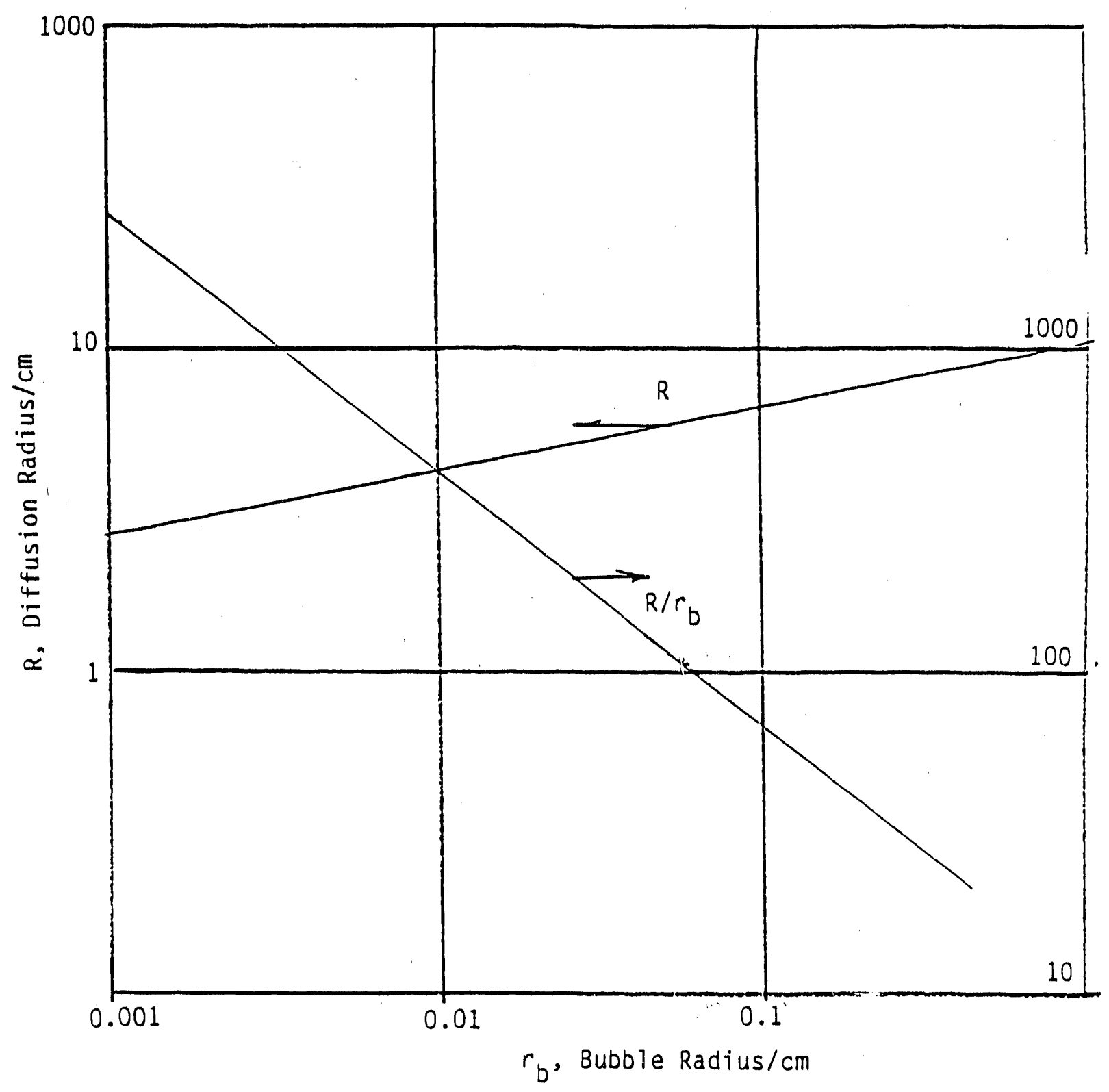

FIGURE B.2. Calculated Diffusion Radius (interbubble half distance) for Various Initial Nucleated Bubble Radii 
APPENDIX C

\section{ESTIMATION OF PARAMETERS}




\section{APPENDIX C}

\section{ESTIMATION OF PARAMETERS}

\section{PRODUCTION RATE}

Based on waste swell and time, this has been estimated as $0.3 \mathrm{~cm}^{3} / \mathrm{L} / \mathrm{day}$. This gives $F=2.76 \mathrm{E}-13 \mathrm{~g} / \mathrm{cm}^{3}$. s for pure hydrogen.

\section{DENSITY IN BUBBLE}

$$
\begin{aligned}
& \rho=\frac{P}{R T} \\
& \rho=\frac{1.1 \cdot 10^{5}}{8315 \cdot 333} \cdot 2=7.4 E^{-5} \mathrm{~g} / \mathrm{cc}
\end{aligned}
$$

where $T=140^{\circ} \mathrm{F}=333 \mathrm{~K}$

$$
\begin{aligned}
& P=1.1 \mathrm{~atm}=1.1 \times 10^{5} \mathrm{~Pa} \\
& R=8315 \frac{\mathrm{Pa} L}{\mathrm{~g}-\mathrm{mole} \mathrm{e}^{\circ \mathrm{K}}}
\end{aligned}
$$

\section{DIFFUSION CONSTANT OF HYDROGEN IN WATER}

From Chemical Rubber Co. Press (1973)

$$
D_{f}=D_{H 2}=5.13 \times 10^{-9} \frac{\mathrm{m}^{2}}{\mathrm{~s}} \approx 5 \times 10^{-5} \frac{\mathrm{cm}^{2}}{\mathrm{~s}}
$$

\section{EQUILIBRIUM CONCENTRATION OUTSIDE OF A BUBBLE}

Pressure in bubble $\approx 1.1 \mathrm{~atm}$.

Henry's law, $P_{g}=H x_{1}$

where $x_{i}=$ mole fraction in liquid. 
Perry and Green "Chemical Engineer's Handbook" 6th Ed. Table 3-133 gives the solubility of hydrogen so $H=7.65 \times 10^{4} \mathrm{~atm} / \mathrm{mole} \mathrm{fr}$.

$$
x=P(a t m) / H
$$

and

$$
C_{E}=2 x / 18=\frac{2}{18} \cdot \frac{1.1}{7.65 \cdot 10^{4}}=1.6 \cdot 10^{-6} \frac{\mathrm{g} \mathrm{H}_{2}}{\mathrm{~mL} \mathrm{H}_{2} \mathrm{O}}
$$

\section{REFERENCE}

Chemical Rubber Co. Press. 1973. Handbook of Appied Engineering Science, 2nd edition, Table 5-45. 
APPENDIX D

ESTIMATION OF HEAT PRODUCTION OF TANK 101-SY 


\section{ESTIMATION OF HEAT PRODUCTION OF TANK 101-SY}

Several methods of calculation are shown in the following pages. The last method given in Table $D .1$ and Figure 2.19 give the best-estimate results shown in Table 0.2 . These values should be helpful in heat transfer calculations and model development.

TABLE D.1. Data from Samples (from Mauss-Sasaki letter dated May 30, 1986)

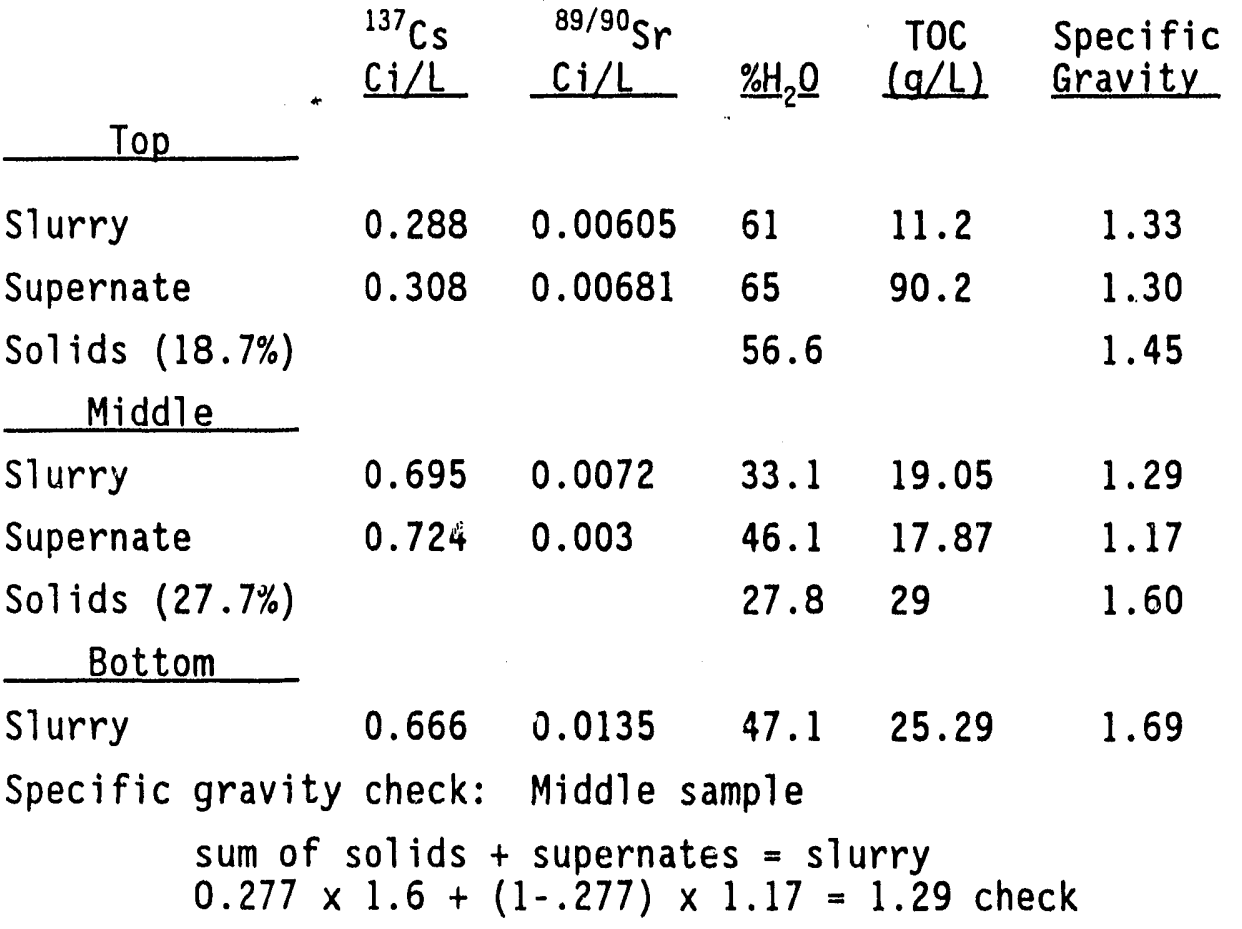

Top Sample

$$
0.187 \times 1.45+(1-.187) \times 1.30=1.33 \text { check }
$$

Method 1

Based on composition estimate of G. P. Simpson letter to L. M. Sasaki August 28, 1987:

Cs $3.102 \times 10^{6}$ Ci $\times 0.0161=49,942$

Sr $2.137 \times 10^{5} \mathrm{Ci} \times 0.0228=\frac{4,872}{54,814} \mathrm{Btu} / \mathrm{h}$

D. 1 
TABLE 0.1. (contd)

\section{Method 2}

Based on Van Tyle letter, March 15, 1990 (essentially the same data as above):

Cs $3.1 M C i \times 0.0161=49,910$

Sr $0.22 M C_{i} \times 0.0228=\frac{5,016}{54,900 \mathrm{Btu} / \mathrm{h}}$

Kelly Corothers says that tank specification is $45,000 \mathrm{Btu} / \mathrm{h}$ for SY tank farms.

Method 3

Corothers' heat calculation method done by hand; notes on a copy of Mauss letter to Sasaki, May 30, 1986.

Typically heating $=1.1 \mathrm{M} \mathrm{gal} \times 3.785(\mathrm{~L} / \mathrm{gal}) \times \mathrm{Aj}(\mathrm{Ci} / \mathrm{L}) \times \mathrm{Bj}(\mathrm{Btu} / \mathrm{Ci} \cdot \mathrm{h})$

\section{Method 4.1}

$$
\begin{aligned}
& B_{C s}=0.0161 \mathrm{Btu} / \mathrm{Ci} \cdot \mathrm{h} \text { from SD - RE-Tl }-131 \\
& B_{S r}=0.0228 \mathrm{Btu} / \mathrm{Ci} \cdot \mathrm{h} \text { from SD }-\mathrm{RE}-\mathrm{Tl}-131
\end{aligned}
$$

If one looks at temperature profile and location of sample, see Figure 2.19, bottom sample appears to represent about 0 to $16.6 \mathrm{ft}$ of tank.

Midsample appears to represent about 16.6 to $31 \mathrm{ft}$ of tank.

Top sample 31 to 34 feet of tank, a region of some temperature change through the crust. The heat from each layer is calculated from the analys is of the mixed slurry sample and the depth it represents.

$$
q_{i}=A_{j} \times B_{j} \times H_{j} \times C
$$

This result could vary a little if the depths associated with each sample were varied, but since the largest depths are associated with the highest $C$ concentration, the result would not change much. This is shown by the last column of Table D.3. If the midsample were taken with the top at $29 \mathrm{ft}$, the $\mathrm{H}$ would be $12.4 \mathrm{ft}$ and the total heat output calculated would be $42,300 \mathrm{Btu} / \mathrm{h}$.

Both results may be more accurate if the sample concentrations were based on a collapsed sample (no gas), whereas the depths associated with each sample were based on measurements of the tank (which is producing gas) and may have the associated volumes, somewhat expanded. Based on a mean expansion of 410 in. $/ 400$ in. $=1.025$ (a $2.5 \%$ expansion) the heat generation values in Table 0.2 could be $2.5 \%$ too large. Correction for this gives the final values in Table D.2.

The bottom layer heat would be

$$
(22,267+639) \times 0.975=22,300 \mathrm{Btu} / \mathrm{h}
$$




\section{TABLE D.1. (contd)}

Method 4.2

Another correction to give an improved estimate of the heat output of the tank is suggested by the temperature profile. In the bottom $4 \mathrm{ft}$, the temperature gradient appears to be constant. This means that little or no heating occurs in this bottom sublayer. The sublayer may be almost solid and have little of the cesium-containing liquid. The depth of the bottom sample seems to be $3 \mathrm{ft}$ above the bottom of the tank. At this level the sludge is still liquid-like so it will have dissolved cesium. Although the bottom sublayer may be like a hard mud and most probably has some liquid in the interstices, the temperature profile suggests that it can be neglected to some extent. Hence, the main heating of the bottom layer would be in the fluid sludge layer of $13.3-\mathrm{ft}$ depth $(16.6$ to $3 \mathrm{ft})$. The sample analys is would be representative of that layer. Then, excluding cesium from the bottom sublayer gives cesium heating in the bottom layer as $18,240 \mathrm{Btu} / \mathrm{h}$. Adding the same strontium heating as in Table 0.2 gives $18,900 \mathrm{Btu} / \mathrm{h}$ in the bottom layer, mostly above the $3-\mathrm{ft}$ level. The total heat from the tank would then be $1,739+52+17,358+254+18,900=38,280 \mathrm{Btu} / \mathrm{h}$. If the $2.5 \%$ correction for expansion is al so made, the total tank heat output would be $37,350 \mathrm{Btu} / \mathrm{h}$ when calculated by Method 4.2.

A revised estimate of the heat output is also needed to reflect the fact that the middle sample was taken from a depth that corresponds to the place within the nonconvecting sludge, although the sample was taken 2 weeks after a release. In this case the concentrations of cesium and strontium for the bottom 1 ayer might be better taken as an average for about an $18-\mathrm{ft}$ height.

This results in a total heat output for the bottom layer of $25,160 \mathrm{Btu} / \mathrm{h}$. The midlayer assumed to be like the supernate of the samples would produce about $17,500 \mathrm{Btu} / \mathrm{h}$ for a total of over $42,000 \mathrm{Btu} / \mathrm{h}$. 
IABLE D.2. Heat Output Calculations, Method 3

\begin{tabular}{|c|c|c|c|}
\hline Top & $A j$ & $B j$ & Btu/h \\
\hline $\begin{array}{l}\text { Slurry Cs } \\
\text { Supern. Cs } \\
\text { Slurry Sr } \\
\text { Supern. Sr }\end{array}$ & $\begin{array}{l}0.288 \\
0.308 \\
0.00605 \\
0.00681\end{array}$ & $\begin{array}{l}0.0161 \\
0.0161 \\
0.0228 \\
0.0228\end{array}$ & $\begin{array}{r}19,305 \\
20,646 \\
574 \\
646 \\
\end{array}$ \\
\hline
\end{tabular}

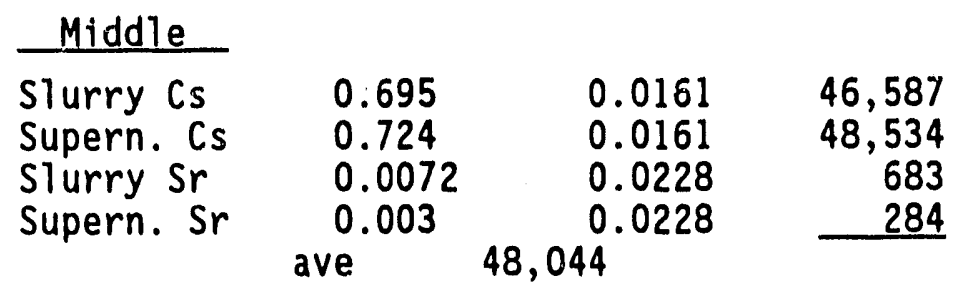

$\begin{array}{cccc}\text { Bottom } & & & \\ \text { Slurry Cs } & 0.666 & 0.0161 & 44,643 \\ \text { Slurry Sr } & 0.0135 & 0.0228 & 1.281 \\ & \begin{array}{ccc}\text { ave } \\ \text { Gross Average } 38,184\end{array} \text { to } & 40,000\end{array}$

This is the value that has been frequently used.

TABLE D.3. Heat Output Calculations, Methods 4.1 and 4.2

$$
(C)^{(a)}
$$

Aj, Ci/L

\begin{tabular}{|c|c|c|c|c|c|c|}
\hline Top & $A j, C i / L$ & $B j, B t u / c c$ & $\underline{H}, \mathrm{ft}$ & i $/ \mathrm{ft}$ & g. Btu/h & q. Btu/h \\
\hline $\begin{array}{l}c_{s}^{s} \\
s_{r}\end{array}$ & $\begin{array}{l}0.288 \\
0.00605\end{array}$ & $\begin{array}{l}0.0161 \\
0.0228\end{array}$ & $\begin{array}{l}3 \\
3\end{array}$ & & $\begin{array}{r}1,739 \\
52\end{array}$ & $\begin{array}{r}1,739 \\
52\end{array}$ \\
\hline
\end{tabular}

\section{Middle}

\begin{tabular}{rrrrrr}
\hline$C_{s}$ & 0.695 & 0.0161 & 15.4 & 21,557 & \\
& & & 12.4 & & 17,358 \\
$S_{r}$ & 0.0072 & 0.0228 & 15.4 & 316 & 254
\end{tabular}

\section{Bottom}

$\begin{array}{rlrrrr}C_{s} & 0.666 & 0.0161 & 16.6 & 22,267 & 22,267 \\ S_{r} & 0.0135 & 0.0228 & 16.6 & \frac{639}{46,570} & \frac{639}{42,309} \\ & & & & 45,405 & 41,250\end{array}$

(a) $\quad c=V_{0} 1 / \mathrm{ft}$ of $\operatorname{tank}=\pi / 4 \times(75)^{2} \mathrm{ft}^{3} / \mathrm{ft} \times 28.317 \mathrm{~L} / \mathrm{ft}^{3}=1.251 \mathrm{E} 5$ $(\mathrm{L} / \mathrm{ft})$. 


\section{DISTRIBUTION}

No. of

Copies

\section{OFFSITE}

12 DOE Office of Scientific and Technical Information

E. C. Ashby 225 North Avenue Boggs Chemistry Building Georgia Institute of Technology Atlanta, GA 30332

N. E. Bibler Westinghouse Savannah River Co.

Bldg 773A, Room B132

Box 616

Aiken, SC 29802

G. R. Chopp in

Department of Chemistry B-164

The Florida State University

Tallahassee, FL 32306

C. Grelecki

Hazards Research Corporation 200 Valley Road, Suite 301

Mt. Arlington, NJ 07856

E. P. Horwitz Chemistry Division

Argonne National Laboratory 9700 Cass Avenue

Argonne, IL 60439-4831

B. R. Kowalski

Chemistry Department BG-10

University of Washington

Seattle, WA 98195

D. Meisel

Chemistry Department

Argonne National Laboratory

9700 Cass Avenue

Argonne, IL 60439-4831
No. of

Copies

F. L. Parker

Vanderbilt University

P.0. Box 1596, Station B

Nashville, TN 37235

G. Powers

Design Science Inc.

163 Witherow Road

Sewickley, PA 15143

G. A. Russe11

Professor of Chemistry

Iowa State University

Gilman Hall

Ames, IA 50011-3111

A. Schneider

Massachusetts Institute of Technology

Department of Nuclear Engineering

Room 24-1098

77 Massachusetts Avenue

Cambridge, MA 02139

W. W. Schulz

727 Sweetleaf Drive

Wilmington, DE 19808

D. D. Siemer

WINCO

IRC MS 2207

Idaho Falls, ID 83403

W. J. Thomson

Dept. of Chemical Engineering Washington State University

Puiliman, WA 99164

J. Tseng

U.S. Department of Energy

EM-35

Trevion II

Washington, DC 20585-0002 
No. of

Copies

G. Wallis

Associate Dean

Thayer School of Engineering

Dartmouth College

Hanover, NH 03755

\section{ONSITE}

3 DOE Richland Field office

R. F. Christensen, A4-02

R. E. Gerton, A5-21

G. W. Rosenwald, A5-21

33 Westinghouse Hanford Company

J. Allen-Floyd, R2-31

H. Babad, B2-15

D. G. Baide, R1-51

M. L. Bel1, T5-50

R. M. Black, R1-19

R. J. BI iss, B3-04

H. R. Brager, L5-03

W. F. Brehm, H5-67

T. M. Burke, $\mathrm{HO}-34$

R. J. Cash, R2-31

D. L. Deichman, HO-03

G. L. Dunford, Rl-51

K. A. Gasper, B3-68

W. H. Hamilton, R2-40

D. L. Herting, T6-50

R. C. Hill, H4-23

J. Jewett, $T 6-50$

G. D. Johnson, L5-03 (2)

N. W. Kirch, R2-11

J. W. Lentsch, R2-31

R. D. Marusich, $\mathrm{H} 5-32$

D. Ogden, $\mathrm{HO}-34$

D. A. Reynolds, R2-11

W. G. Ruff, R2-53

M. H. Shannon, B1-35

D. D. Stepnewski, $\mathrm{Nl}-31$

R. E. Vandercook, S6-07

D. D. Wodrich, R2-23
No. of

Copies

43 Pacific Northwest Laboratory

R. T. Al lemann, K5-19 (5)

Z. I. Antoniak, K7-15

J. A. Bamberger, $\mathrm{K} 7-15$

J. M. Bates, K7-15

J. B. Colson, $K 5-10$

T. H. Dunning, K2-18

J. R. Friley, K5-22

C. E. Haines, K7-28

R. T. Hallen, K2-12

M. S. Hanson, Kl-51

B. M. Johnson, Jr., K5-02

M. R. Kreiter, KE-35 (5)

D. K. Lemon, K2-28

L. M. Liljegren, K7-15

G. B. Mellinger, K1-78

L. G. Morgan, P8-07

L. R. Pederson, K2-44

J.T.A. Roberts, K1-73

R. D. Scheele, P7-25

S. Somasundaram, K5-19

J. C. Spanner, K2-31

D. M. Strachan, K2-44

R. W. Stromatt, P7-22

D. S. Trent, K1-82

H. H. Van Tuy1, P7-22

Publishing Coordination

Technical Report Files (5) 

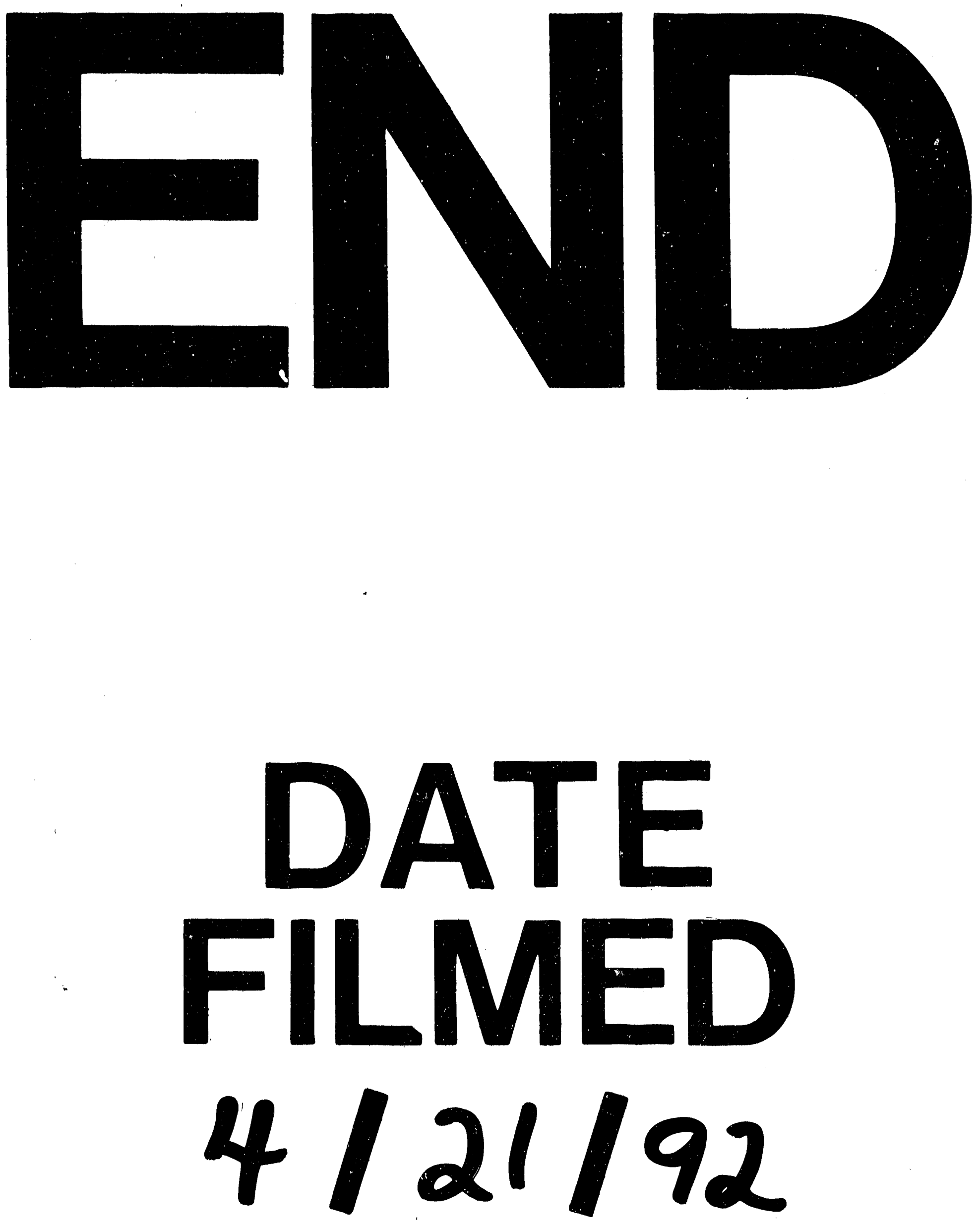


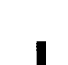

